University of Tennessee Health Science Center

UTHSC Digital Commons

\title{
A Novel Elvitegravir Nanoformulation for Drug Delivery Across the Blood-Brain Barrier to Suppress HIV-1 in Macrophages and Microglia
}

Yuqing Gong

University of Tennessee Health Science Center

Follow this and additional works at: https://dc.uthsc.edu/dissertations

Part of the Pharmacy and Pharmaceutical Sciences Commons

\section{Recommended Citation}

Gong, Yuqing (https://orcid.org/0000-0001-6373-5440), "A Novel Elvitegravir Nanoformulation for Drug Delivery Across the Blood-Brain Barrier to Suppress HIV-1 in Macrophages and Microglia" (2020). Theses and Dissertations (ETD). Paper 512. http://dx.doi.org/10.21007/etd.cghs.2020.0497. 


\title{
A Novel Elvitegravir Nanoformulation for Drug Delivery Across the Blood-Brain Barrier to Suppress HIV-1 in Macrophages and Microglia
}

\author{
Abstract \\ Over the last two decades, the use of antiretroviral therapy (ART) has remarkably decreased the morbidity \\ associated with HIV-1 infection. However, the prevalence of HIV-1-associated neurocognitive disorders \\ (HAND) is still increasing. The appearance and persistence of HAND are partially due to the entry of \\ HIV-1-infected monocytes into the brain. Within the central nervous system (CNS), HIV-1-infected \\ macrophages and microglia serve as the major viral reservoirs and provide active viral replication even \\ when systemic viral suppression has been achieved by ART. Efficient viral suppression of CNS \\ macrophages and microglia is important for an effective HIV-1 treatment in the brain. The primary \\ hindrance to the treatment of HAND is mainly contributed by the inability of antiretrovirals (ARVs) to cross \\ the blood-brain barrier (BBB) after the systemic administration. Conventional regimens of ARVs are not \\ sufficient to penetrate the BBB or improve outcomes in HAND. Thus, there is a need for novel treatment \\ regimens that cross the BBB and deliver therapeutic ARVs into the CNS to suppress the HIV-1 replication \\ in these viral reservoirs. Our objective in this study was to improve the efficacy of ART in CNS HIV-1 \\ reservoirs, specifically in macrophages and microglia. Our central hypothesis was that the selected ARV, \\ elvitegravir (EVG), in nanoformulation would have increased transmigration across the BBB, as well as \\ increased drug uptake in macrophages and microglia after crossing the BBB, leading to increased \\ antiviral activity in macrophages and microglia, relative to the native drug. This study was expected to \\ provide an optimized treatment strategy that has potential for therapeutic interventions in reducing \\ HAND.In this study, poly (lactic-co-glycolic acid) (PLGA)-based elvitegravir nanoparticles (PLGA-EVG NPs) \\ were prepared by nano-precipitation. Firstly, we analyzed the physicochemical properties of PLGA-EVG \\ NPs using transmission electron microscopy, dynamic light scattering (DLS), and Fourier-transform \\ infrared spectroscopy (FTIR). We measured cellular uptake of PLGA NPs by fluorescence microscopy and \\ flow cytometry. We also measured the intracellular drug concentration and viral replication in \\ HIV-1-infected macrophages by using LC-MS/MS and p24 ELISA, respectively. The PLGA-E

\section{Document Type \\ Dissertation} \\ Degree Name \\ Doctor of Philosophy $(\mathrm{PhD})$ \\ Program \\ Pharmaceutical Sciences \\ Research Advisor \\ Santosh Kumar, Ph.D. \\ Keywords \\ Blood-brain barrier, Elvitegravir, HIV-associated neurocognitive disorder, Macrophages, Microglia, \\ Nanoparticle \\ Subject Categories \\ Medicine and Health Sciences | Pharmacy and Pharmaceutical Sciences
}




\section{A Novel Elvitegravir Nanoformulation for Drug Delivery Across the Blood-Brain Barrier to Suppress HIV-1 in Macrophages and Microglia}

Author:

Yuqing Gong
Advisor:

Santosh Kumar PhD

A Dissertation Presented for The Graduate Studies Council of

The University of Tennessee Health Science Center

in Partial Fulfillment of the Requirements for the

Doctor of Philosophy degree from

The University of Tennessee

in

Biomedical Engineering/Biomechanics

College of Graduate Health Sciences

May 2020 
Portions of Chapters 3 and 4 (c) 2017 by Elsevier B.V. All other material $\odot 2020$ by Yuqing Gong. All rights reserved. 


\section{DEDICATION}

I dedicate this dissertation to my beloved parents,

Mr. Li Gong and Mrs. Hong Mao,

for their support and love during my graduate study. 


\section{ACKNOWLEDGEMENTS}

I would like to express my deepest appreciation to my mentor, Dr. Santosh Kumar for his support, encouragement and guidance. During my graduate training, he taught me not only science but also the philosophy to become a good person. His guidance helped me grow and move forward during my graduate study.

I would also like to extend my deepest gratitude to my co-mentor, Dr. Murali M. Yallapu for his guidance, practical suggestions, and support for this project. This project cannot move smoothly without the support from him. I am also grateful to my other committee members, Dr. Theodore J. Cory, Dr. Sarka Beranova, and Dr. Francesca-Fang Liao, for the thoughtful comments and recommendations on this dissertation.

I would like to thank the department of Pharmaceutical Sciences for the financial support to my graduate study. I also thank to National Institutes of Health for providing grants to Dr. Santosh Kumar (AA022063 and DA047178) and Dr. Murali M. Yallapu (K22CA174841).

Many thanks to my lab mates and my friends for their support during my graduate study. Lastly, special thanks to my parents and my family members for their love. 


\begin{abstract}
Over the last two decades, the use of antiretroviral therapy (ART) has remarkably decreased the morbidity associated with HIV-1 infection. However, the prevalence of HIV-1-associated neurocognitive disorders (HAND) is still increasing. The appearance and persistence of HAND are partially due to the entry of HIV-1-infected monocytes into the brain. Within the central nervous system (CNS), HIV-1-infected macrophages and microglia serve as the major viral reservoirs and provide active viral replication even when systemic viral suppression has been achieved by ART. Efficient viral suppression of CNS macrophages and microglia is important for an effective HIV-1 treatment in the brain. The primary hindrance to the treatment of HAND is mainly contributed by the inability of antiretrovirals (ARVs) to cross the blood-brain barrier (BBB) after the systemic administration. Conventional regimens of ARVs are not sufficient to penetrate the BBB or improve outcomes in HAND. Thus, there is a need for novel treatment regimens that cross the $\mathrm{BBB}$ and deliver therapeutic ARVs into the CNS to suppress the HIV-1 replication in these viral reservoirs.
\end{abstract}

Our objective in this study was to improve the efficacy of ART in CNS HIV-1 reservoirs, specifically in macrophages and microglia. Our central hypothesis was that the selected ARV, elvitegravir (EVG), in nanoformulation would have increased transmigration across the $\mathrm{BBB}$, as well as increased drug uptake in macrophages and microglia after crossing the $\mathrm{BBB}$, leading to increased antiviral activity in macrophages and microglia, relative to the native drug. This study was expected to provide an optimized treatment strategy that has potential for therapeutic interventions in reducing HAND.

In this study, poly (lactic-co-glycolic acid) (PLGA)-based elvitegravir nanoparticles (PLGA-EVG NPs) were prepared by nano-precipitation. Firstly, we analyzed the physicochemical properties of PLGA-EVG NPs using transmission electron microscopy, dynamic light scattering (DLS), and Fourier-transform infrared spectroscopy (FTIR). We measured cellular uptake of PLGA NPs by fluorescence microscopy and flow cytometry. We also measured the intracellular drug concentration and viral replication in HIV-1-infected macrophages by using LC-MS/MS and p24 ELISA, respectively. The PLGA-EVG NPs showed an average particle size of $\sim 47 \mathrm{~nm}$ from transmission electron microscopy and a zeta potential of - $6.74 \mathrm{mV}$ from DLS. These PLGA NPs demonstrated a time- and concentration-dependent uptakes in monocytes. PLGA-EVG NPs showed a $\sim 2$ times higher intracellular internalization of EVG than the native drug in HIV-1-infected monocytic cell line. PLGA-EVG NPs also demonstrated superior viral suppression to native drug for a prolonged period of time in human primary monocyte-derived macrophages.

Secondly, we quantified the stability, human serum interaction, hemocompatibility, cyto-compatibility, and cellular internalization of the PLGA-EVG NPs. The stability of PLGA NPs was assessed by measurement of particle size and zeta potential of NPs under different storage conditions. The human serum interaction with 
NPs was assessed using FTIR, DLS, and sodium dodecyl sulfate polyacrylamide gel electrophoresis (SDS-PAGE) analysis. The biocompatibility of PLGA-EVG NPs was studied with red blood cells (RBCs), macrophages, and microglia. We also studied the endocytosis pathway and the sub-cellular localization of PLGA-NPs in macrophages and microglia using endocytosis inhibitors and sub-cellular localization markers. These characterizations suggest a favorable safety and stability profile of EVG nanoformulation for use as a potential CNS delivery strategy. PLGA NPs were stable at room temperature for at least a week and at $4{ }^{\circ} \mathrm{C}$ and $-20^{\circ} \mathrm{C}$ for at least 30 days with no change in size or zeta potential. The PLGA-EVG NPs also showed a good biocompatibility with RBCs, macrophages, and microglia. Human serum protein binding occurs on PLGA NPs with upon incubation with human serum. The protein corona formed on PLGA NPs did not significantly alter the particle size, hemocompatibility, or biocompatibility of PLGAEVG NP to human primary monocyte-derived macrophages and microglia. The internalization of PLGA NPs in macrophages involves to a combination of endocytosis pathways, while the main internalization of PLGA NPs in microglia occurred through caveolae-/clathrin-mediated endocytosis. PLGA NPs can escape from endo-lysosomal compartments and deliver the therapeutics to the cells efficiently.

Thirdly, we assessed the ability of PLGA-EVG NPs to cross the BBB and suppress the HIV-1 replication in macrophage and/or microglial cells. The penetration of EVG native drug /nanoformulation was determined using an in vitro BBB model and validated with immunodeficient NSG mice. The efficacy of HIV-1 suppression in the CNS was assessed using an in vitro BBB model utilizing HIV-1-infected human primary monocyte-derived macrophages and microglia and validated with an HIV-1 encephalitic (HIVE) mouse model. Compared with EVG native drug, the PLGA-EVG NPs demonstrated improved BBB penetration in both in vitro and in vivo systems. Most importantly, the PLGA-EVG NPs were able to show enhanced HIV-1 suppression in HIV-1-infected human monocyte-derived macrophages and monocyte-derived microglia after crossing the BBB, without altering BBB integrity. PLGA-EVG NPs also showed a trend of decreasing CNS HIV-1 viral load in HIV-1-infected mice. Overall, this is an innovative and optimized treatment strategy that has a potential for therapeutic interventions in reducing HAND. 


\section{TABLE OF CONTENTS}

CHAPTER 1. INTRODUCTION ...............................................................................

Introduction of HIV-1-Associated Neurocognitive Disorder (HAND) .........................

Summary and Limitations of Current Treatment Strategies for HAND .........................

Approaches of Antiretrovirals (ARVs) to Cross the Blood-Brain Barrier (BBB)...........

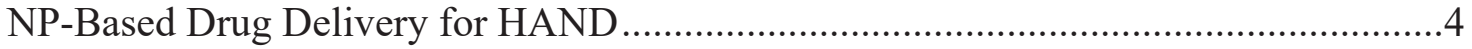

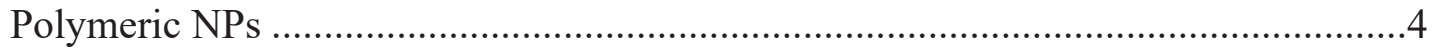

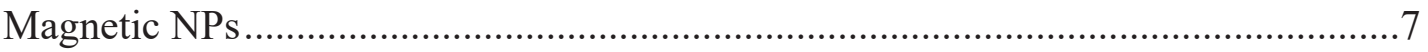

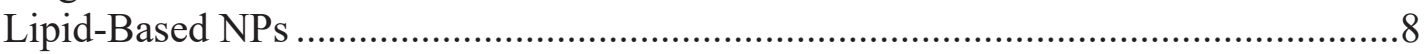

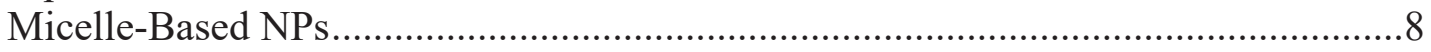

Drug Delivery Strategy Involving PLGA-NPs and Elvitegravir (EVG) .....................9

\section{CHAPTER 2. SCIENTIFIC PREMISE, HYPOTHESIS, OBJECTIVE AND}

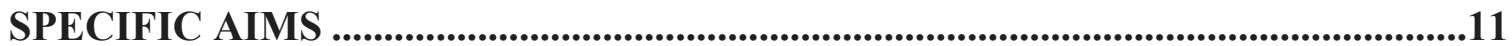

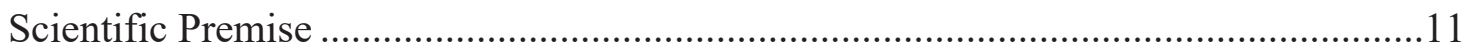

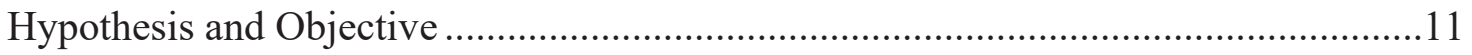

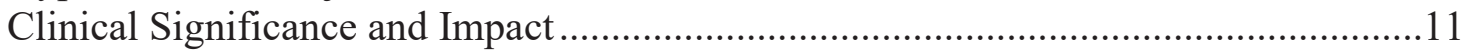

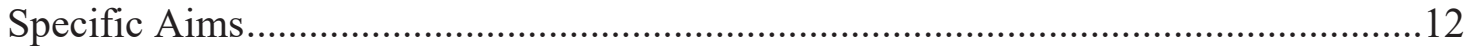

Aim 1: Synthesize, Characterize, and Determine Pharmacokinetic Profile and

Efficacy of the PLGA-based NP for the Potential Use of Delivery EVG to

Macrophages

Aim 2: Determine the Stability, Protein Corona, Biocompatibility, and Cellular Internalization Pathway of PLGA-EVG NPs

Aim 3: Assess the Ability of PLGA-EVG NPs to Cross the BBB and the Improvement of Viral Suppression in HIV-1-Infected Macrophages and

Microglia after BBB Transmigration.....

CHAPTER 3. MATERIALS AND METHODS.........................................................14

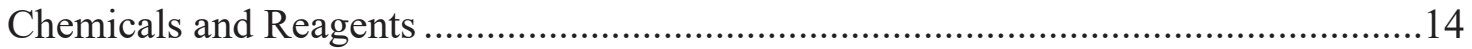

Experimental Methods .............................................................................................. 15

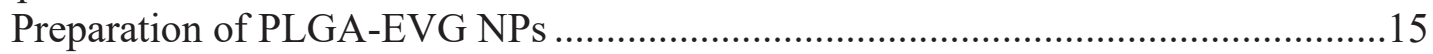

Physiochemical Characterization of PLGA-EVG NPs ...................................... 15

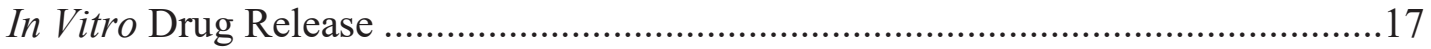

Cellular Uptake of PLGA NPs in Monocytes ...................................................... 17

Generation of Human Monocytes-Derived Macrophages and Monocytes-

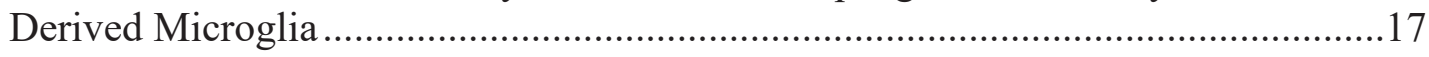

HIV-1 Infection of Macrophages and Microglia ................................................ 18

Intracellular EVG Concentration and Viral Suppression of PLGA-EVG in HIV-

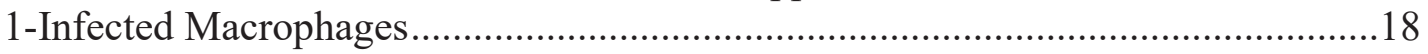

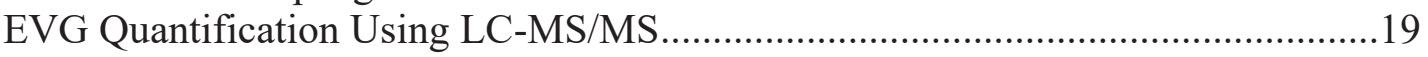

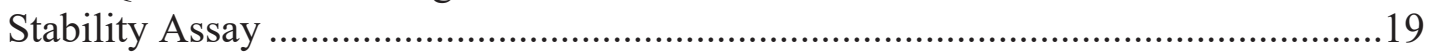

Human Serum Protein Binding to PLGA NPs......................................................20

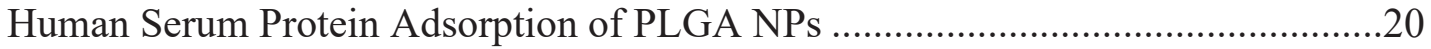

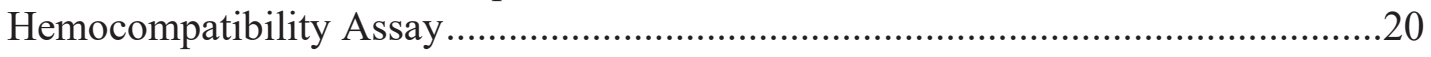


Biocompatibility Assay with Macrophages and Microglia.........................................21

Sub-Cellular Localization in Macrophages and Microglia .........................................21

Cellular Uptake and Internalization Mechanism in Macrophages and Microglia .....22

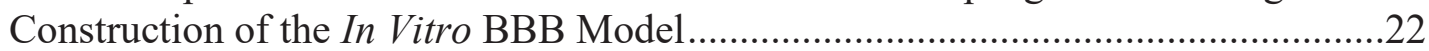

Transendothelial Electrical Resistance Measurements ............................................22

In Vitro BBB Transmigration Study of PLGA NPs................................................23

Penetration of PLGA-EVG NPs in the In Vitro BBB Model ...................................23

Mechanistic Contribution of P-gp in Opposing the Penetration of EVG Across

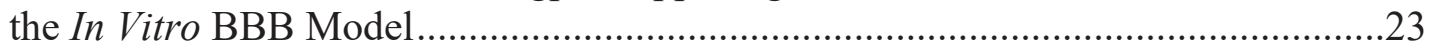

EVG Levels in the In Vivo Mouse Model.............................................................24

Viral Suppression of PLGA-EVG NPs in HIV-1-Infected Macrophages and

Microglia after Crossing the In Vitro BBB Model .................................................24

Viral Suppression of PLGA-EVG NPs in HIV-1 Encephalitic (HIVE) Mice............25

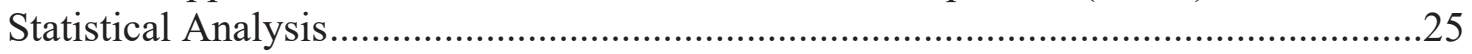

CHAPTER 4. RESULTS AND DISCUSSION ...............................................................26

Novel EVG Nanoformulation Approach to Suppress the Viral Load in HIV-1-

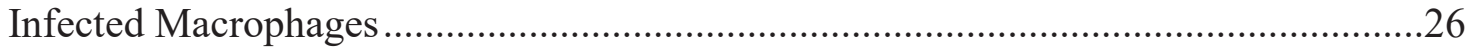

Physiochemical Characterization of PLGA-EVG NPs ............................................26

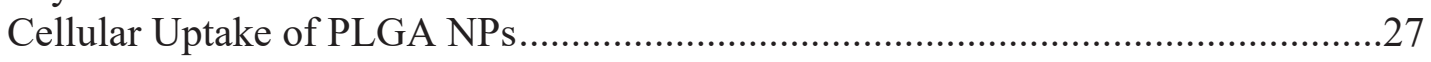

Intracellular EVG Concentration and Viral Suppression Efficacy of PLGA-EVG

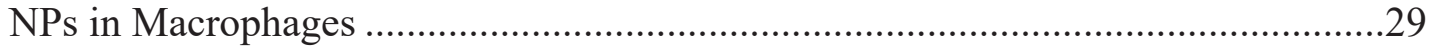

Stability, Protein Corona, Biocompatibility, and Cellular Internalization Pathway

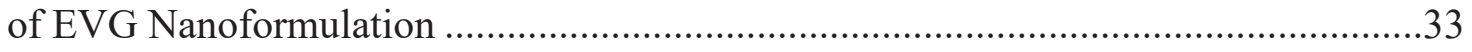

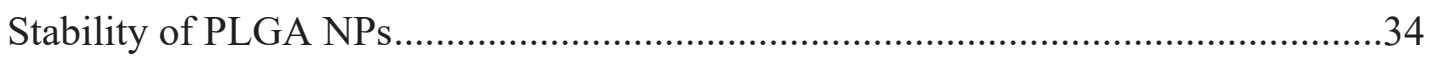

Evaluation of Human Serum Protein Binding on PLGA NPs ......................................34

Hemocompatibility and Biocompatibility of PLGA-EVG NPs...................................37

Internalization Mechanism of PLGA NPs in Macrophages and Microglia ................40

EVG Nanoformulation for Drug Delivery Across the BBB to Achieve HIV-1

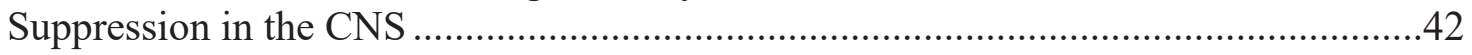

In Vitro BBB Model and Transendothelial Electrical Resistance Assessments ........42

Transmigration of PLGA NPs Across the In Vitro BBB ........................................44

Increased Penetration of PLGA-EVG NPs in the In Vitro BBB Model and In Vivo Mouse Model ............................................................................................

Improved Viral Suppression in HIV-1-Infected Macrophages and Microglia after

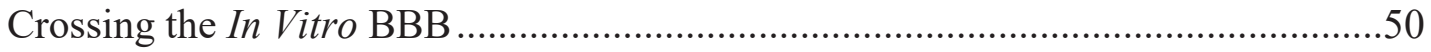

CHAPTER 5. CONCLUSIONS AND FUTURE DIRECTIONS .................................54

LIST OF REFERENCES .................................................................................................58

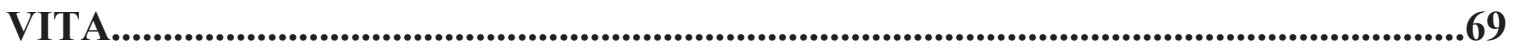




\section{LIST OF TABLES}

Table 1-1. Summary of NPs of ARVs used for CNS delivery......................................5

Table 4-1. Size and zeta potential of PLGA NPs stored at room temperature, $4{ }^{\circ} \mathrm{C}$

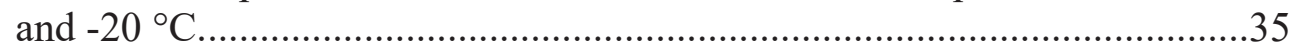

Table 4-2. $\quad$ Size and zeta potential of PLGA NPs in 1mM HEPES buffer from $\mathrm{pH} 1$

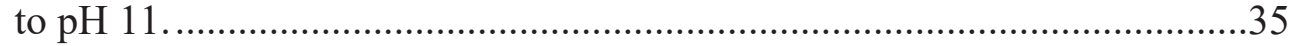




\section{LIST OF FIGURES}

Figure 1-1. The development of HAND and the use of EVG nanoformulation across the BBB to suppress the CNS HIV-1 viral replication...................................10

Figure 2-1. Overall strategies of developing PLGA-EVG NPs for drug delivery across the BBB to suppress HIV-1 in macrophages and microglia.

Figure 3-1. A hypothetical structural representation of the PLGA-EVG NP..................16

Figure 4-1. Physiochemical characterization of PLGA-EVG NPs..................................28

Figure 4-2. Cellular uptake of nanoparticles. ……………..........................................

Figure 4-3. Intracellular EVG concentration in U1 cells...............................................31

Figure 4-4. Viral suppression of PLGA-EVG NPs. ......................................................

Figure 4-5. Evaluation of human serum protein binding on PLGA NPs..........................36

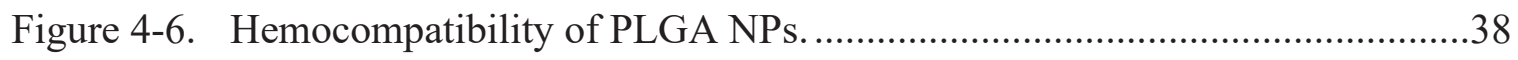

Figure 4-7. Toxicity evaluation of PLGA-EVG NPs in MDM and MMG. ....................39

Figure 4-8. Internalization mechanism of the PLGA-C6 NPs in MDM and MMG........41

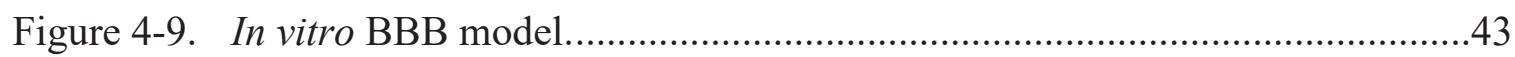

Figure 4-10. Dose dependent penetration of PLGA NPs across the BBB in vitro.............45

Figure 4-11. Dose-/time-dependent penetration of EVG and PLGA-EVG NPs across

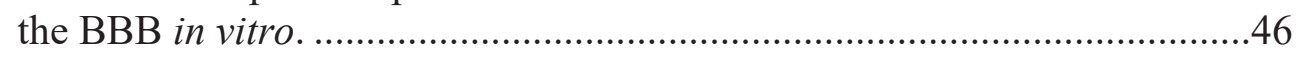

Figure 4-12. Plasma and brain concentrations of EVG and PLGA-EVG NPs in mice.....49

Figure 4-13. Viral suppression of EVG and PLGA-EVG in HIV-1-infected MDM after crossing an in vitro BBB model........................................................51

Figure 4-14. Viral suppression of EVG and PLGA-EVG in HIV-1-infected MMG after crossing an in vitro BBB model...........................................................53

Figure 5-1. Viral suppression of EVG and PLGA-EVG in a HIVE mouse model. ........56 


\section{LIST OF ABBREVIATIONS}

$\begin{array}{ll}\text { ART } & \text { Antiretroviral therapy } \\ \text { ARV } & \text { Antiretrovirals } \\ \text { BBB } & \text { Blood-brain barrier } \\ \text { BCRP } & \text { Breast cancer resistance protein } \\ \text { CNS } & \text { Central nervous system } \\ \text { Cobi } & \text { Cobicistat } \\ \text { CPE } & \text { CNS penetration-effectiveness } \\ \text { CSF } & \text { Cerebrospinal fluid } \\ \text { DLS } & \text { Dynamic light scattering } \\ \text { EVG } & \text { Elvitegravir } \\ \text { FDA } & \text { Food and Drug Administration } \\ \text { FIs } & \text { Fusion inhibitors } \\ \text { FTIR } & \text { Fourier-transform infrared spectroscopy } \\ \text { HAND } & \text { HIV-1-associated neurocognitive disorders } \\ \text { HIVE } & \text { HIV-1 encephalitic } \\ \text { INSTIs } & \text { Integrase strand transfer inhibitors } \\ \text { MNPs } & \text { Magnetic nanoparticles } \\ \text { MRP1 } & \text { Multidrug resistance associated protein } \\ \text { NNRTIs } & \text { Non-nucleotide reverse transcriptase inhibitors } \\ \text { NRTIs } & \text { Nucleotide reverse transcriptase inhibitors } \\ \text { PBCA } & \text { Poly(butyl-cyanoacrylate) } \\ \text { PGA } & \text { Poly(glycolic acid) } \\ \text { P-gp } & \text { P-glycoprotein } \\ \text { PIs } & \text { Protease inhibitors } \\ \text { PLA } & \text { Poly(lactic acid) } \\ \text { PLGA } & \text { Poly(lactic-co-glycolic acid) } \\ \text { PLGA-EVG NPs } \quad \text { Poly(lactic-co-glycolic acid)-based elvitegravir nanoparticles } \\ \text { RBCs } & \text { Red blood cells } \\ \text { RTV } & \text { Ritonavir } \\ \text { SDS-PAGE } & \text { Sodium dodecyl sulfate polyacrylamide gel electrophoresis } \\ \text { SLN } & \text { Solid lipid nanoparticles } \\ & \end{array}$




\section{CHAPTER 1. INTRODUCTION}

\section{Introduction of HIV-1-Associated Neurocognitive Disorder (HAND)}

In the past three decades, the introduction of antiretroviral therapy (ART) has led to significant advances in the effectively management of HIV-1/AIDS [1]. However, the prevalence of HIV-1-associated neurocognitive disorders (HAND) is still a major concern, especially in the aging population [2]. Around 15-55\% of HIV-1-infected individuals are estimated to have HAND in the ART era, which remains the same proportion as was reported prior to the widespread use of ART [3]. Although HIV-1associated dementia, the most severe form of HAND, is rare in developed countries due to the use of ART, the incidence of asymptomatic neurocognitive impairment and minor neurocognitive disorders remains unresolved [2]. The appearance and persistence of HAND are mainly due to the entry of HIV-1 virus into the brain via the "Trojan horse" mechanism through infected monocytes and macrophages [4, 5]. Monocytes and macrophages can be infected by HIV-1 and can function as viral reservoirs because they are capable of surviving with HIV-1 infection for an extended period of time [6]. These HIV-1-infected monocytes/macrophages infiltrate the brain and spread the virus to resident macrophages and microglia in the central nervous system (CNS) [7]. Further, HIV-1 actively replicates in macrophages and microglia, providing persistent viral replication in the brain $[8,9]$. Since macrophages and microglia are located in the brain parenchyma where neurons and glia connect extensively, HIV-1 has significant opportunity to interact with neurons and uninfected glial cells [10]. Although neurons are not directly infected by HIV-1 virus, active replication of HIV-1 in infected macrophages and microglia produces toxic components, including viral proteins and inflammatory cytokines and chemokines, which damage neurons and ultimately cause HAND [11, 12]. Individuals with HAND commonly show characteristics of dysfunctional and impaired judgment, memory, multitasking, and attention [13]. Additionally, HIV-1 infection in the brain affects not only cognitive functioning but also the opportunity for HIV-1 eradication [2]. The brain can serve as a viral reservoir for the production of HIV-1, and provide active viral replication even when systemic viral suppression has been achieved [14]. Currently, ART is the best option for delaying or preventing HIV-1-associated disease progression [15]. However, due to the inability of antiretroviral drugs (ARVs) to efficiently cross the blood-brain barrier (BBB) following systemic administration, ART is still not clinically applicable for the treatment of HAND [2]. Thus, there is a need for novel treatment regimens that cross the BBB and deliver therapeutics into the brain to suppress viral replication in CNS cells. Discovering a new delivery system to maximize the treatment outcomes of HAND has become an important research avenue in HIV-1 therapy.

\section{Summary and Limitations of Current Treatment Strategies for HAND}

In 1987, the United States (US) Food and Drug Administration (FDA) licensed the first ARV zidovudine (also known as azidothymidine) [16]. Zidovudine works by 
inhibiting reverse transcriptase enzyme of HIV-1, slowing the replication of the virus and the progression of the disease. In the last three decades, ART has made a significant contribution to the reduction in HIV/AIDS-related death and improved quality of life for people living with HIV/AIDS [17]. In 2018, there were more than 30 ARVs approved for the treatment of HIV-1 in the US [18]. ARVs are classified into six groups according to the mechanism of action: nucleotide reverse transcriptase inhibitors (NRTIs), nonnucleotide reverse transcriptase inhibitors (NNRTIs), protease inhibitors (PIs), fusion inhibitors (FIs), entry inhibitors (also known as CCR5 antagonists), and integrase strand transfer inhibitors (INSTIs) [19]. ARVs can be used either as a single-drug regimen or a combination of 3-4 drugs [20]. In most cases, in order to achieve highest efficacy, ARVs are used in combination regimens that can attack multiple stages of the HIV-1 life cycle, and subsequently slow the disease progression.

However, ARVs are not used as a specific treatment strategy for HAND. Many ARVs have restricted entry into the CNS across the BBB after systemic administration [21]. The BBB includes endothelial cells, pericytes, perivascular astrocytes, and tight junctions, and forms an almost impermeable cellular barrier that restricts the free diffusion of compounds into the CNS $[22,23]$. In addition to the physical barrier, the poor drug penetration is partly due to the active efflux mediated by membrane-associated transporters located at the BBB. In particular, ATP-binding cassette (ABC) membraneassociated transporters, including P-glycoprotein (P-gp), breast cancer resistance protein (BCRP), and multidrug resistance associated protein (MRP1) are significantly expressed at the BBB [24]. As a result, the BBB only selectively transports molecules such as certain amino acids, sugars, and hydrophilic molecules (e.g. oxygen and carbon dioxide) into the brain. In addition, co-administration of ARVs with strong CYP3A4 inhibitors such as cobicistat (cobi) and ritonavir (RTV) has been used to enhance the efficacy of some ART regimens [25]. However, most ARVs show limited CNS penetration relative to plasma drug concentration even with ritonavir (RTV) boosting [22, 26]. For example, RTV boosting is capable of increasing indinavir plasma level significantly but not cerebrospinal fluid (CSF) indinavir concentration [26]. When boosted with RTV, atazanavir and lopinavir reach therapeutic concentrations in plasma, but remain undetectable in CSF [22]. Previous clinical studies have demonstrated that the CNS penetration-effectiveness (CPE) score of ARVs is correlated with the inhibition of HIV-1 replication in the CNS and superior cognitive performance in HIV-1-positive patients $[27,28]$. While several NRTIs and NNRTIs such as zidovudine and efavirenz showed good CPE scores that penetrate the CNS well, high CNS concentrations of those ARVs are neurotoxic and are potentially associated with the development of HAND [22]. Thus, to achieve neurological benefit, the ideal therapeutic agent should achieve optimal CNS penetration with minimal neurotoxicity.

Other than treating HAND through viral suppression, researchers are searching for adjunctive therapies to treat the neurological and psychological secondary symptoms, using anti-depressants, anti-psychotics, and anti-anxiety drugs [29]. Several neuroprotective agents including memantine, selegiline, sodium valproate, paroxetine, and fluconazole have been investigated as potential adjunctive therapies for patients with HAND [29, 30]. However, prior trials of adjunctive therapies have not consistently 
shown clinical benefit in cognitive improvement in HAND patients [30]. Additionally, the CNS can still serve as a latent HIV-1 reservoir with adjunctive therapies because those drugs do not reduce viral replication and treat the root cause of HAND [30]. Specific developments in therapeutic agents that cross the BBB and suppress viral replication are needed for the treatment of HIV-1 in the CNS.

\section{Approaches of Antiretrovirals (ARVs) to Cross the Blood-Brain Barrier (BBB)}

Several approaches, such as blocking ATP-binding cassette (ABC) transporters, BBB opening strategy, prodrug therapy, and nanoparticle-based drug delivery, have been employed to improve the penetration of ARVs [31]. Most ARVs, especially PIs, are ABC transporter substrates and have limited brain penetration due to efflux transportation [31]. Using LY-335979, a P-gp inhibitor, to downregulate the P-gp expression increased the brain concentrations of nelfinavir, amprenavir, indinavir, and saquinavir up to 37-fold, while only modestly increased plasma drug levels, causing an increase in brain-plasma ratios from 14-fold to 17 -fold in mdrla $(+/+)$ mice [32]. Other studies have also shown similar results with saquinavir and indinavir using P-gp inhibitors (quinidine and GF 120918) and an MRP1 inhibitor (MK571) in in vitro BBB models and in vivo mice models [33,34]. Although blocking ABC transporters may significantly improve drug concentrations in the brain, this method is not clinically achievable as a treatment approach for HAND because the inhibition of ABC transporters in other organs may cause serious drug-drug interactions [31]. Another approach to increasing drug penetration to the brain is the "BBB opening" approach. Opening the BBB can be achieved by using a hyper-osmotic solution to shrink the endothelial cells or using certain cytotoxic agents to disrupt the BBB tight junctions $[35,36]$. However, opening the tight junctions of the BBB is not recommended because it may also allow the entry of the virus and other contaminants into the brain [31]. Development of prodrugs of ARVs to increase their capacity to penetrate the BBB is another potential delivery approach [31]. Prodrugs can be synthesized with sufficient lipophilicity to allow them to cross the endothelial cell membrane and release the parent ARVs into the brain. Tenofovir disoproxil fumarate, a prodrug of tenofovir, has shown the capability of crossing the blood-CSF barrier to reach the HIV-1 infected perivascular and meningeal macrophages. Unfortunately, it was not able to reach the deep brain, so there was no drug accumulation within HIV-1-infected microglia [37]. Since prodrugs are considered to be a separate chemical entity, the approval process for prodrugs is as complicated and difficult as for the parent molecule, [31]. Developing prodrugs as a delivery approach needs a full evaluation of toxicity, cost, and efficacy.

Compared with other CNS delivery approaches, nanoparticle (NP)-based drug delivery approaches are an attractive option for the treatment of HAND [38]. NP-based formulations are a colloidal system that made of polymers, lipids, or a combination of both [39]. A therapeutic agent is either encapsulated in the core of the colloid matrix or adsorbed entrapped on the surface of the particle. Because of their colloidal structure, an NP-based drug delivery system can protect ARVs from efflux transporters and both enzymatic and hydrolytic degradations, with a potential for controlled release [40]. NPs 
can cross the BBB through membrane transcytosis and effectively deliver the therapeutic molecule to the CNS. Thus, a therapeutic molecule can be loaded on a nanoparticle which has good interactions with the endothelial cells at the BBB and increases the drug's distribution throughout the brain [39].

\section{NP-Based Drug Delivery for HAND}

NPs that have been studied for brain delivery mainly include polymeric NPs such as poly(glycolic acid) (PGA), poly(lactic acid) (PLA), poly(D, L-lactide-co-glycolide) (PLGA), and poly(butyl-cyanoacrylate) (PBCA) NPs [41, 42]; magnetic NPs (MNPs) composed of an iron oxide core [42]; lipid-based nanoformulation such as solid lipid nanoparticles (SLN) and liposomes; and micelle-based nanoformulations such as Pluronic micelles [31]. As a proof-of-concept, preclinical studies have used NP-based delivery systems for transport of ARVs across the BBB to suppress HIV-1 in the CNS (Table 1-1).

\section{Polymeric NPs}

Since polymeric NPs are not only easily prepared and stored, but also highly biocompatible and biodegradable in nature, they have been extensively studied for delivery of therapeutics for cancer [43-46] and HIV-1 [47-50]. Several groups have demonstrated the capability of polymeric NPs to deliver ARVs to the brain for the treatment of HAND. Kou et al. have investigated the transmigration of ARVs including stavudine, delavirdine, and saquinavir across an in vitro BBB by using PBCA NPs and methylmethacrylate-sulfopropylmethacrylate (MMA-SPM) NPs [51]. The permeability of those selected ARVs across human brain microvascular endothelial cells (HBMEC) can be improved by 12- to 16-fold with PBCA NPs, and 3- to 7-fold with MMA-SPM NPs. They also observed that a decrease in the BBB permeability is associated with an increase in the particle size of NPs for these three ARVs. The same group further modified the surface of the MMA-SPM NPs with a synthetic linear pseudopeptide, RMP7 , for targeted delivery of stavudine, delavirdine, and saquinavir across the BBB [52]. In this study, they used a polymerization initiator, ammonium persulfate, to generate uniform and spherical RMP-7-MMA-SPM NPs. They found that the RMP-7-conjugated NPs were able to mediate the opening of the tight junctions and enhance endocytosis by the BBB, improving the permeability of these three ARVs into the brain. They also investigated the use of PBCA NPs and MMA-SPM NPs with other ARVs such as zidovudine and lamivudine [53]. Using the bovine brain endothelial cell line BBMEC as an in vitro model of the $\mathrm{BBB}, \mathrm{PBCA}$ NPs were able to improve the $\mathrm{BBB}$ permeability of zidovudine and lamivudine by up to 20 and 18 folds, respectively. MMA-SPM NPs were able to improve the BBB permeability of zidovudine and lamivudine by 1 fold [53]. In addition, Mainardes et al developed PLA NPs and PLA-polyethylene glycol (PEG) blend NPs containing zidovudine [54]. The PLA NPs and PLA-PEG NPs showed mean diameters of $265.8 \mathrm{~nm}$ and $328.1 \mathrm{~nm}$, respectively. Although they did not study the brain permeability of the NPs directly, they showed the PLA-PEG blend NPs have the capacity 
Table 1-1. Summary of NPs of ARVs used for CNS delivery.

\begin{tabular}{|c|c|c|c|c|c|c|}
\hline NP type & Particle materials & ARVs & Size (nm) & $\begin{array}{l}\text { Improvement of BBB } \\
\text { transmigration (compared with } \\
\text { native drug) }\end{array}$ & Tested model & Ref. \\
\hline $\begin{array}{l}\text { Polymeric } \\
\text { NPs }\end{array}$ & PBCA & $\begin{array}{l}\text { stavudine, } \\
\text { delavirdine, } \\
\text { saquinavir }\end{array}$ & 184 & $\uparrow 12-16$ fold & HBMEC cells & [51] \\
\hline $\begin{array}{l}\text { Polymeric } \\
\text { NPs }\end{array}$ & MMA-SPM & $\begin{array}{l}\text { stavudine, } \\
\text { delavirdine, } \\
\text { saquinavir }\end{array}$ & 68 & $\uparrow 3-7$ fold & HBMEC cells & [51] \\
\hline $\begin{array}{l}\text { Polymeric } \\
\text { NPs }\end{array}$ & $\begin{array}{l}\text { RMP-7/MMA- } \\
\text { SPM }\end{array}$ & $\begin{array}{l}\text { stavudine, } \\
\text { delavirdine, } \\
\text { saquinavir }\end{array}$ & 50 & - & HBMEC cells & [52] \\
\hline $\begin{array}{l}\text { Polymeric } \\
\text { NPs }\end{array}$ & PBCA & $\begin{array}{l}\text { zidovudine, } \\
\text { lamivudine }\end{array}$ & 150 & $\begin{array}{l}\uparrow 20 \text { fold, } \\
\uparrow 18 \text { fold }\end{array}$ & BBMEC cells & [53] \\
\hline $\begin{array}{l}\text { Polymeric } \\
\text { NPs }\end{array}$ & MMA-SPM & $\begin{array}{l}\text { zidovudine, } \\
\text { lamivudine }\end{array}$ & 25 & $\uparrow 1$ fold & BBMEC cells & [53] \\
\hline $\begin{array}{l}\text { Polymeric } \\
\text { NPs }\end{array}$ & PLA & zidovudine & 265.8 & - & - & [54] \\
\hline $\begin{array}{l}\text { Polymeric } \\
\text { NPs }\end{array}$ & PLA-PEG & zidovudine & 328.1 & - & - & [54] \\
\hline $\begin{array}{l}\text { Magnetic } \\
\text { NPs }\end{array}$ & $\begin{array}{l}\mathrm{Fe} 3 \mathrm{O} 4, \\
\text { phosphatidyl } \\
\text { choline, } \\
\text { cholesterol }\end{array}$ & azidothymidine & 150 & $\uparrow 3$ fold & BMVEC cells & [55] \\
\hline $\begin{array}{l}\text { Magnetic } \\
\text { NPs }\end{array}$ & $\mathrm{Fe} 3 \mathrm{O} 4$, vorinostat & tenofovir & $10 \pm 3$ & $\uparrow 2$ fold & HBMEC cells & [56] \\
\hline
\end{tabular}


Table 1-1. (Continued).

\begin{tabular}{|c|c|c|c|c|c|c|}
\hline NP type & Particle materials & ARVs & Size (nm) & $\begin{array}{l}\text { Improvement of BBB } \\
\text { transmigration (compared with } \\
\text { native drug) }\end{array}$ & Tested model & Ref. \\
\hline $\begin{array}{l}\text { Magnetic } \\
\text { NPs }\end{array}$ & $\begin{array}{l}\mathrm{Fe} 3 \mathrm{O} 4, \\
\text { PEG, transferrin } \\
\text { antibody, } \\
\text { phophatidyl } \\
\text { choline, } \\
\text { cholesterol }\end{array}$ & $\begin{array}{l}\text { nelfinavir, } \\
\text { tenofovir }\end{array}$ & $410 \pm 15$ & $\begin{array}{l}\uparrow 50-100 \% \text { (compared to MNPs } \\
\text { without transferrin conjugation) }\end{array}$ & HBMEC cells & $\begin{array}{l}{[57]} \\
{[58]}\end{array}$ \\
\hline $\begin{array}{l}\text { Magnetic } \\
\text { NPs }\end{array}$ & $\mathrm{CoFe} 2 \mathrm{O} 4, \mathrm{BaTiO} 3$ & azidothymidine & $19-31$ & $\uparrow 3$ fold & HBMEC cells & [59] \\
\hline $\begin{array}{l}\text { Magnetic } \\
\text { NPs }\end{array}$ & $\begin{array}{l}\mathrm{Fe} 3 \mathrm{O} 4, \mathrm{Au}, \\
\text { DPPC, cholesterol, } \\
\text { PEG }\end{array}$ & $\begin{array}{l}\text { tenofovir } \\
\text { disoproxil } \\
\text { fumarate }\end{array}$ & $\sim 100$ & $\uparrow 1$ fold & CHME-5 cells & [60] \\
\hline $\begin{array}{l}\text { Lipid- } \\
\text { based NPs }\end{array}$ & $\begin{array}{l}\text { hydrogenated } \\
\text { castor oil }\end{array}$ & darunavir & 200 & $\uparrow 3$ fold & $\begin{array}{l}\text { male Wistar } \\
\text { rats }\end{array}$ & $\begin{array}{l}{[61,} \\
62]\end{array}$ \\
\hline $\begin{array}{l}\text { Lipid- } \\
\text { based NPs }\end{array}$ & flaxseed oil & saquinavir & $100-200$ & $\uparrow 5$ fold & $\begin{array}{l}\text { male Balb/c } \\
\text { mice }\end{array}$ & [63] \\
\hline $\begin{array}{c}\text { Micelle- } \\
\text { based NPs }\end{array}$ & Pluronic 407 & dolutegravir & $300-400$ & No neurotoxicity & $\begin{array}{l}\text { male } \mathrm{Balb} / \mathrm{c} \\
\text { mice }\end{array}$ & [64] \\
\hline
\end{tabular}

Notes: PBCS- polybutylcyanoacrylate, MMA-SPM- methyl methacrylate-sulfopropyl methacrylate, PLA- poly (lactic acid), DPPC1,2-dipalmitoyl-sn-glycero-3-phosphocholine, PEG- polyethylene glycol. - Data not available. 
to avoid the phagocytosis, staying in the systemic circulation for longer time, and can be used as a potential delivery method to transport zidovudine into the brain.

\section{Magnetic NPs}

In order to deliver therapeutics to the brain more specifically, MNPs which can be controlled by an external magnetic field are used [65]. MNPs transport through the BBB followed the external magnetic force, which allows the control of movement and speed. Saiyed et al. from the Nair group developed a MNPs of an NRTI (azidothymidine) encapsulated into liposomes made with a mixture of phosphatidyl choline and cholesterol [55]. These magnetic liposomes can be taken up by monocytes, which further enhances the BBB transmigration. These magnetic liposomes showed 3-fold higher transmigration across an in vitro BBB model, compared to the free drug, following application of an external magnetic field. The magnetic liposomes of azidothymidine also showed slightly lower p24 antigen production then free drug, when treated to HIV-1-infected human peripheral blood mononuclear cells (PBMCs) [55]. Jayant et al. from the same group also demonstrated the development of a layer-by-layer (LbL) MNP to achieve sustained drug release in the brain [56]. The magnetic LbL NP was made with an HIV-1 NRTI (tenofovir) and a latency-breaking agent (vorinostat). The magnetic LbL NP showed a $37.95 \%$ BBB transmigration and 33\% p24 suppression on primary astrocytes in vitro after 5 days of the initial dose [56].

The same group also attempted to encapsulate MNPs into PEGylated liposomes conjugated with anti-transferrin antibody to target transferrin receptor-mediated transportation through the BBB [57]. These transferrin-targeted MNPs showed a 50$100 \%$ increase in drug transmigration across the in vitro BBB compared with MNPs without transferrin. A recent study from the Nair group combined the use of LbL MNPs and transferrin-targeted MNPs, developing a transferrin-conjugated LbL MNP of nelfinavir and tenofovir [58]. The transferrin-conjugated LbL MNPs were able to transmigrate $\sim 15 \%$ of MNPs across the in vitro BBB. These MNPs provided a 10-day sustained release profile and continually suppressed HIV-1 virus up to $\sim 50 \%$ in HIV-1infected human astrocytes. In addition, Nair et al. developed a magneto-electric nanoparticle (MENPs) to exhibit both magnetic and electric property of the NP for the delivery of azidothymidine [59]. Because of the dual effect from the magnetic field and the electric force, the movement and drug release of MENPs can be remotely controlled by manipulating those fields. These MENPs were enabled to transmigrate $\sim 40 \%$ azidothymidine across the $\mathrm{BBB}$ in the in vitro $\mathrm{BBB}$ within 6 hours, while free drug only showed $\sim 10 \%$ transmigration. A higher HIV-1 p24 viral suppression was also observed in PBMCs by using the MENPs with no cytotoxicity.

Other than the Nair group, Tomitaka et al. developed magneto-plasmonic liposomes (MPLs) of tenofovir disoproxil fumarate [60]. The MPLs are made of sodium citrate coated MNPs, coated with Au and polyethylene glycol, then encapsulated into a liposome that was made of 1,2-dipalmitoyl-sn-glycero-3-phosphocholine (DPPC) and cholesterol. One-fold higher transmigration of MPLs was observed in the presence of an 
external magnetic field and the MPLs also provided a greater $\mathrm{p} 24$ reduction compared with free tenofovir disoproxil fumarate.

\section{Lipid-Based NPs}

Lipid-based NPs are made of a wide range of biological lipids and have shown potential for delivery of ARVs to the CNS [31]. Due to their lipophilic structures, lipidbased NPs naturally show high affinity for the BBB. Desai et al. developed an SLN of the HIV-1 PI darunavir, which enhanced the oral bioavailability by $\sim 4$-fold in comparison to native darunavir obtained from the rats' pharmacokinetics data [61]. The authors have clearly demonstrated both advantages of SLN and of active targeting of the drugs to reach the HIV reservoir $[61,62]$. They showed that the improvement in oral bioavailability could be attributed to darunavir entrapping in the lipid matrix, resulting in enhanced lymphatic uptake of NPs and absorption over the native drug [61]. The same group also developed NPs loaded with darunavir and grafted the surface with a peptide sequence (CARRPKFYRAPYVKNHPNVWG) that has an affinity for CD4 receptors on the HIV host cells [62]. The authors reported an enhancement in transport of SLNs loaded with darunavir in Caco- 2 cells, which mimics the gastrointestinal membrane, by 4-fold in contrast to free darunavir, due to increased endocytosis. Further, these SLNs were able to penetrate the $\mathrm{BBB}$ and have 3 -fold increased accumulation in the brain in comparison to native darunavir suspension [62]. In addition, Vyas et al. prepared a flaxseed oil-based nanoemulsion of saquinavir for brain delivery [63]. The size of the saquinavir-containing nanoemulsion is in the range of 100-200 $\mathrm{nm}$. When dosed orally, the nanoemulsion showed a 3-fold higher saquinavir systemic exposure compared with native drug. Importantly, the nanoemulsion significantly altered the drug distribution in the brain. The maximum concentration of saquinavir was found to be 5-fold higher in the brain, and the area under the curve (AUC) of the drug was found to be 3 -fold higher in the male Balb/c mice brain.

\section{Micelle-Based NPs}

A micelle-based NP is an amphiphilic, self-assembling polymeric NP which has a hydrophobic core to encapsulate poor water-soluble drugs [66]. Due to their amphiphilic structure, micelle-based NPs have a hydrophilic exterior surface that provides optimized aqueous solubility. For example, Montenegro-Burke et al. from the Gendelman group demonstrated the use of Pluronic micelles to achieve a long-acting slow release of an ARV, dolutegravir [64]. They used Pluronic 407 to prepare a P407-dolutegravir micelles with particle size of 300-400 $\mathrm{nm}$. They observed a large quantity of oxidative stressrelated metabolites in brain subregions when using the native doltegravir, suggesting the potential neurotoxicity and incompatibility of doltegravir as a therapeutic method for the intervention of HAND. However, negligible oxidative stress-related metabolites were observed when doltegravir is encapsulated in the Pluronic 407 NP. This study demonstrated the potential use of micelle-based NPs to minimize the neurotoxicity of doltegravir for the treatment of HIV-1 in the CNS. 
In summary, although NP-based delivery systems of ARVs for CNS delivery are still limited to preclinical studies, recent studies have shown the potential of NPs to cross the BBB and combat HIV-1 infection in the CNS [31]. Size, charge, and surface modification of NPs all can contribute to successful CNS delivery. NPs with a size smaller than $200 \mathrm{~nm}$ have a better chance to cross the BBB via transcytosis through clathrin-mediated endocytosis. Since the endothelial cell layer is negatively charged, positively charged NPs can enter the BBB through adsorptive transcytosis, which is easier than neutral or negatively charged NPs. On the other hand, neutral and negatively charged NPs also have benefits because of their reduced protein adsorption, leading to a longer circulation period. NPs can also be modified with ligands to target transport- or receptor-mediated transcytosis, such as via albumin transporters and glucose transporter 1 (GLUT1) [31]. Overall, NP-based delivery systems are likely to provide a new approach for delivery of ARVs for HAND therapy. Since NP-based delivery systems are a novel delivery method, in order to move forward toward clinical use, comprehensive toxicity and safety assessments of NPs need to be performed, including assessment of long-term adverse reactions, neurotoxicity, accumulation of nanomaterials, and their efficacy after release inside the CNS.

\section{Drug Delivery Strategy Involving PLGA-NPs and Elvitegravir (EVG)}

HIV-1 enters brain via HIV-1-infected monocytes and macrophages and spreads the virus in CNS cells especially perivascular macrophages and microglia $[8,9,67]$. Due to the active replication of HIV-1 in these CNS reservoirs, toxic components including viral proteins and inflammatory cytokines and chemokines are produced, leading to immune activation, inflammation, and neuronal damage $[11,12]$. In our study, we selected elvitegravir (EVG) as our therapeutic molecules, which is an FDA-approved INSTI, and is part of the first line ART regimen for the newly infected HIV-1 populations in the USA [68]. Mohideen et al. formulated EVG into PLA NPs for protection against HIV-1 [69]. The surface of this PLA NPs was formed with hyperbranched polyglycerols (HPG) to create bio-adhensive NPs. EVG showed a prolonged retention on C57BL/6 mice vaginal lumen compared with free drug. Moreover, Mandel et al. from the Destache group developed a PLGA-based NP containing EVG and tenofovir alafenamide for subcutaneous delivery as a prevention strategy against HIV [70]. The size of NP was $190.2 \mathrm{~nm}$ with zeta potential of $-19.2 \mathrm{mV}$. A detectable drug level was found from with the NP group for 14 days, while the free drug showed a detectable drug level for $72 \mathrm{~h}$. This EVG NPs were able to protect the mice with $100 \%$ and $60 \%$ of uninfected rate on day 4 and day 14, respectively, after HIV challenge. The half-life of EVG was also improved from $11 \mathrm{~h}$ to 3.3 days in humanized CD34+ NSG mice [71].

We selected EVG as the therapeutic molecule in our nanoformulation because EVG has a better safety profile than other classes of ARVs [72], which would make it more appropriate to use as a CNS therapy approach. EVG has limited BBB penetration, showed as low as $\sim 0.3 \%$ CSF-plasma drug concentration ratio in HIV-infected individuals [73]. To improve efficacy of EVG, we decided to encapsulate the drug into a 
NP formulation to increase its transmigration across the BBB, and increased drug uptake in macrophages and microglia after entry into the CNS. With regards to NPs, we selected PLGA-NP delivery system because it is a biodegradable and FDA-approved material for human clinical applications [74]. PLGA NPs have been shown to have improved delivery of therapeutic molecules across the BBB with safe and minimal/tolerable off-site toxicity to cells [75]. PLGA NPs also have been shown to have the ability to encapsulate multiple drugs, including ART drugs, which is important for treating HIV-1, as single drug regimen for HIV-1 is not sufficient to effectively suppress HIV-1 [70]. A schematic graph of the overall strategy for this study is showed in Figure 1-1.

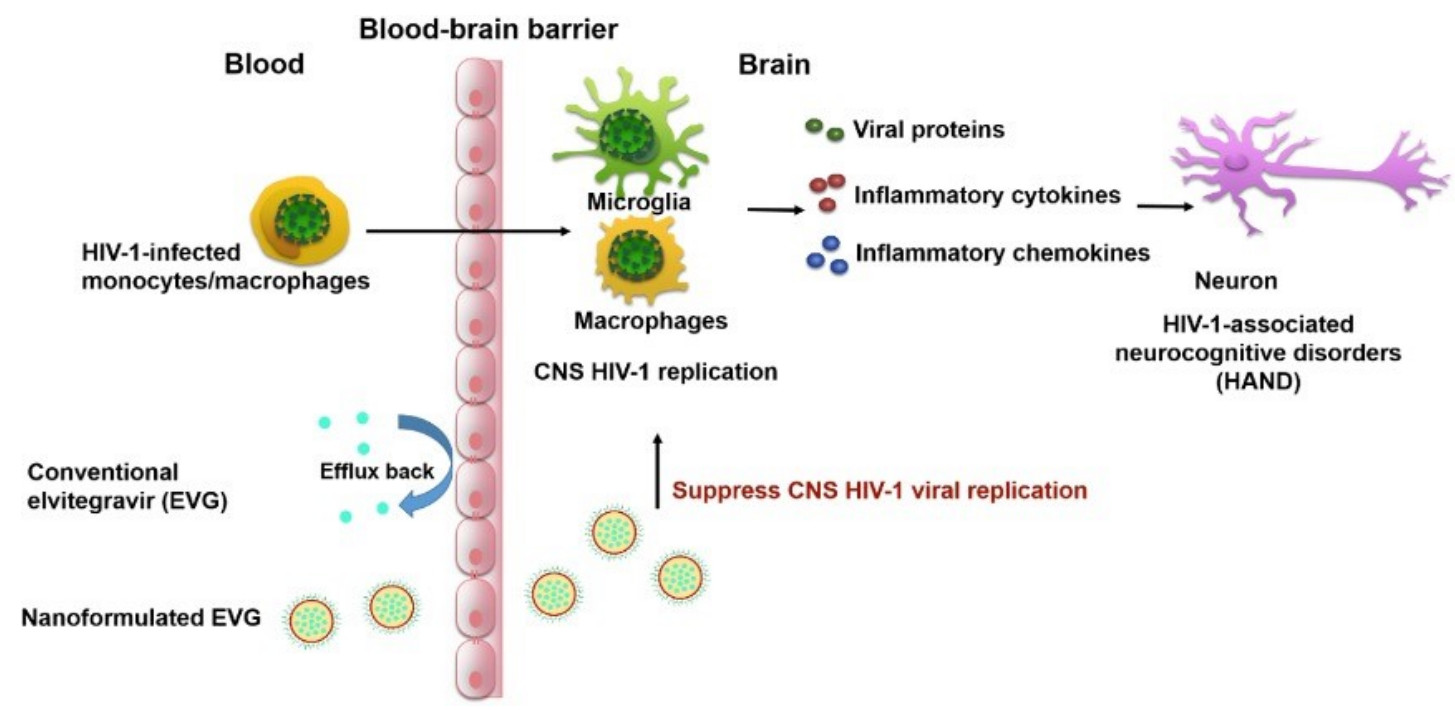

Figure 1-1. The development of HAND and the use of EVG nanoformulation across the BBB to suppress the CNS HIV-1 viral replication. 


\section{CHAPTER 2. SCIENTIFIC PREMISE, HYPOTHESIS, OBJECTIVE AND SPECIFIC AIMS}

\section{Scientific Premise}

The scientific premise of this project is derived from literature reports and our own studies: 1) HIV-1 can infiltrate the brain through monocytes and actively replicate in brain macrophage and microglia [8, 9, 67]. 2) At least 25\% of ART-treated HIV-1 patients developed one or more neurological syndromes [76]. 3) HIV-1 in the brain is difficult to eliminate by current ART, primarily as a result of their suboptimal ARV concentrations [21]. 4) The suboptimal drug concentrations in the brain can be explained by the inability of ARVs to cross the BBB after systemic administration [21]. 5) The drug efflux transporters P-gp and MRP1, and the drug-metabolizing enzyme cytochrome $\mathrm{p} 450$ 3A4 (CYP3A4), are significantly expressed in both macrophages and microglia, making it more difficult to maintain therapeutic drug levels in these cells [77-79]. 6) The literature shows that NPs are generally taken up by cells more efficiently than native drug are [38]. 7) The brain permeability of a drug can be optimized by NP-based drug delivery systems [38]. 8) Due to the rapid in vivo degradation and the minimal possibility of NP accumulation in the brain, NPs made with biocompatible polymers are suitable for brain delivery [39]. 9) Poly(lactic-co-glycolic acid) (PLGA) is a biocompatible polyester that can be converted into water and carbon dioxide via the tricarboxylic acid cycle and eventually eliminated from the body [74]. The literature shows that poloxamer 188 coating can help PLGA NPs deliver therapeutics more efficiently into the brain [80].

\section{Hypothesis and Objective}

Our central hypothesis is that the selected ARV, elvitegravir (EVG), in a poloxamer188-PLGA-based NP will have increased transmigration across the BBB, and increased drug uptake in macrophages and microglia after crossing the $\mathrm{BBB}$, leading to increased antiviral activity in those cells, relative to the free drug. Our objective in this study is to improve the viral suppression efficacy of EVG in CNS HIV-1 reservoirs, especially macrophages and microglia, and to provide an optimized treatment strategy that has a potential for therapeutic interventions in reducing HAND.

\section{Clinical Significance and Impact}

This project provides the basis of scientific knowledge and technical capability of using a poloxamer188-PLGA-based NP as a new delivery modality for the effective treatment of HIV-1 infection in viral reservoirs in the brain, such as macrophages and microglia. This project demonstrates the potential use of NPs to provide a more favorable drug pharmacokinetics, greater viral suppression efficacy, and a superior safety profile when compared with free drug molecules. When the aims of this project are achieved, the concepts, methods, technologies, and potential interventions related to the application of 
PLGA-based NP that target macrophages and microglia would provide a necessary base for the effective treatment of HAND.

\section{Specific Aims}

\section{Aim 1: Synthesize, Characterize, and Determine Pharmacokinetic Profile and Efficacy of the PLGA-based NP for the Potential Use of Delivery EVG to Macrophages}

We will synthesize a poloxomar188-PLGA based NP (PLGA-NP) formulation with EVG (PLGA-EVG NPs). We will analyze the physicochemical properties of PLGANPs using transmission electron microscopy [54], dynamic light scattering (DLS), and Fourier-transform infrared spectroscopy (FTIR). We will measure the cellular uptake of PLGA-NPs using flow cytometry. We will also measure the intracellular drug concentration and viral replication in macrophages by using LC-MS/MS and p24 ELISA, respectively. We will obtain the physicochemical properties and cellular internalization of PLGA NPs to show their potential use of increasing drug concentrations in macrophages through Aim 1.

\section{Aim 2: Determine the Stability, Protein Corona, Biocompatibility, and Cellular Internalization Pathway of PLGA-EVG NPs}

We will identify the particle size and zeta potential of PLGA NPs under different storage conditions. We will assess the human serum protein adsorption of PLGA NPs using FTIR, DLS, and sodium dodecyl sulfate polyacrylamide gel electrophoresis (SDSPAGE) analysis. We will identify the biocompatibility of PLGA-EVG NPs with red blood cells (RBCs), macrophages, and microglia. We will also study the endocytosis pathway and the sub-cellular localization of PLGA-NPs in macrophages and microglia using endocytosis inhibitors and sub-cellular localization markers.

\section{Aim 3: Assess the Ability of PLGA-EVG NPs to Cross the BBB and the Improvement of Viral Suppression in HIV-1-Infected Macrophages and Microglia after BBB Transmigration}

We will perform transmigration assays of PLGA-NPs on both the in vitro BBB model and in vivo mouse model. We will calculate the percentage of transendothelial migration of EVG in EVG-loaded NPs and in free drug form. We will also assess viral suppression in HIV-1-infected macrophages and microglia after crossing the BBB, and we will also use an HIV-1 encephalitic (HIVE) mice model to validate the results. The overall strategies for specific aims are showed in Figure 2-1. 


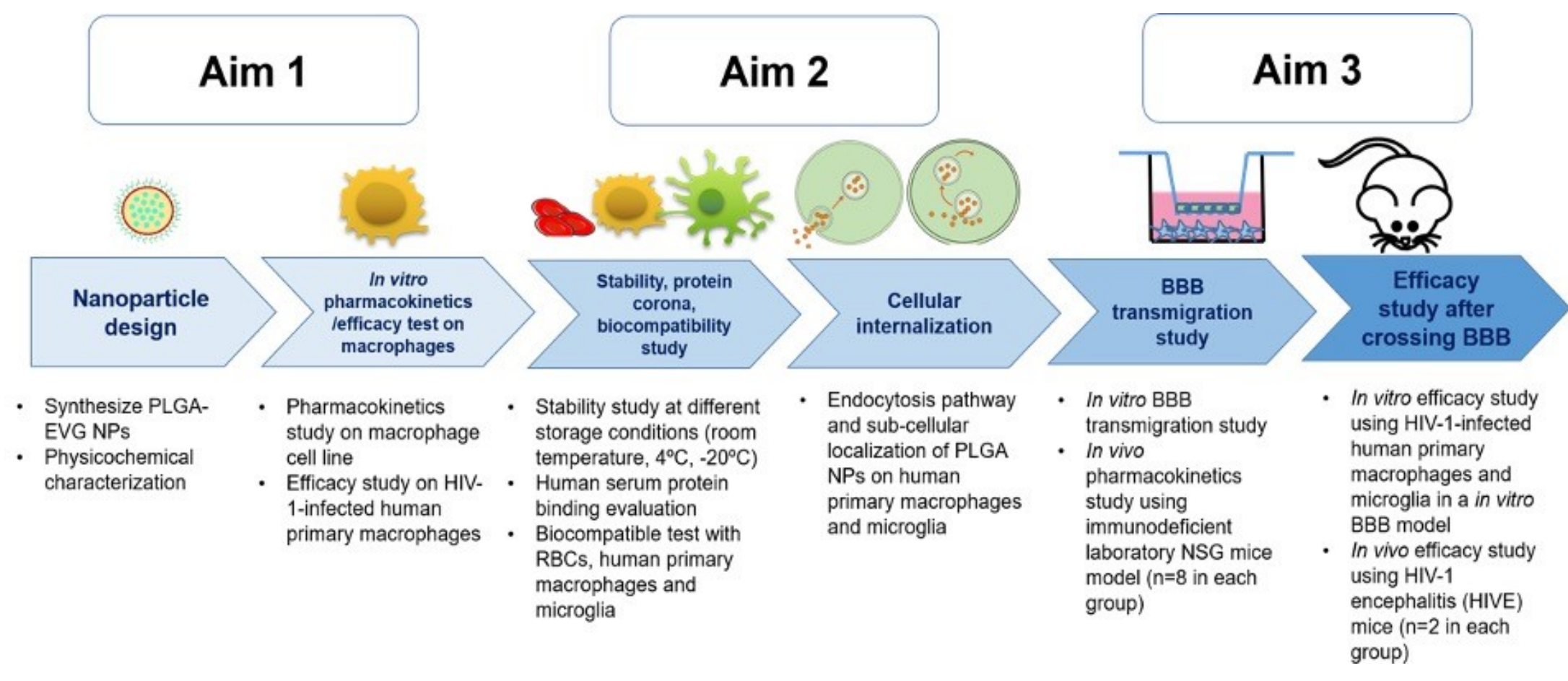

Figure 2-1. Overall strategies of developing PLGA-EVG NPs for drug delivery across the BBB to suppress HIV-1 in macrophages and microglia. 


\section{CHAPTER 3. MATERIALS AND METHODS ${ }^{1}$}

\section{Chemicals and Reagents}

Elvitegravir (EVG, E509000) was purchased from Toronto Research Chemicals Inc. (Ontario, Canada). Poly(D,L-lactide-co-glycolide) (PLGA) (50:50 lactide-glycolide ratio, Mw: 31,000-50,000, ester-terminated) was brought from Birmingham Polymers (Pelham, AL). The following items were obtained from Sigma-Aldrich Co. (St. Louis, MO): poloxamer 188 (pluronic F-68) (P1300, Mw: 8350), poly(vinyl alcohol) (PVA) (363138, Mw: 30,000-70,000), poly(L-lysine) (PLL) (Mw: 30,000-70,000), coumarin-6 (442631), acetone (650501), nocodazole (M1404), cytochalasin-D (Cyto-D) (C8273), chlorpromazine (CPZ) (C1240), monensin sodium salt (M5273), genistein (G6649), methyl- $\beta$-cyclodextrin (M $\beta$-CD) (C4555), hexadimethrine bromide (polybrene) (107689), MitoTracker ${ }^{\mathrm{TM}}$ Deep Red (M22426), Texas Red $^{\mathrm{TM}}$ Conjugate (T2875), CellLight ${ }^{\mathrm{TM}}$ Late Endosomes-RFP (C10589), and Terg-a-zyme ${ }^{\circledR}$ enzyme detergent (Z273287). HPLC grade acetonitrile (A955) and formic acid (85178), BD PrecisionGlide 25G needle (14826-49) and BD $1 \mathrm{~mL}$ TB syringe (14-826-88) were obtained from Fisher Scientific (Hampton, NH). Sterile phosphate-buffered saline was obtained from Gibco (Dublin, Ireland). Ethylenediaminetetraacetic acid (EDTA) was purchased from Boston Bio Products (Ashland, MA).

Dulbecco's Modified Eagle's Medium (DMEM) was obtained from American Type Culture Collection (Manassas, VA, USA). Roswell Park Memorial Institute (RPMI) 1640 media and lymphocyte separation medium were bought from Corning Inc (Tewksbury, MA). Fetal bovine serum (FBS) was obtained from Atlanta Biologicals, Inc. (Atlanta, GA). Ammonium-chloride-potassium lysing buffer were obtained from Life Technologies (Carlsbad, CA). L-glutamine and penicillin-streptomycin (P/S) were purchased from Fisher Scientific. The human recombinant cytokines, including M-CSF, GM-CSF, beta-nerve growth factor (NGF- $\beta$ ), and CCL2 used for macrophage/microglia differentiation were purchased from PeproTech (Rocky Hill, NJ), Recombinant Human IL-2 Protein was purchased from R\&D Systems, Inc. (Minneapolis, MN). HIV-1 Ada strain was obtained from the NIH AIDS Reagent Program (Germantown, MD). RosetteSep enrichment cocktail was purchased from StemCell Technologies (Vancouver, Canada). The p24 ELISA kit (801111) was purchased from ZeptoMetrix Corporation (Buffalo, NY) to assess HIV-1 viral load in HIV-1-infected macrophages and microglia.

${ }^{1}$ Portions of chapter reprinted from final submission with permission. Gong, Y., et al. (2017). Novel elvitegravir nanoformulation approach to suppress the viral load in HIVinfected macrophages. Biochemistry and Biophysics Reports, 2017. 12: p. 214-219. DOI: https://doi.org/10.1016/j.bbrep.2017.10.005 [81]. Portions of chapter reprinted from final submission with open access permission. Gong, Y., et al. (2020) Novel elvitegravir nanoformulation for drug delivery across the blood-brain barrier to achieve HIV-1 suppression in the CNS macrophages. Sci Rep 2020. 10, 3835. DOI:

https://doi.org/10.1038/s41598-020-60684-1 [82]. 


\section{Experimental Methods}

\section{Preparation of PLGA-EVG NPs}

The PLGA-EVG NP formulation was prepared by nano-precipitation as described in our previous study [83]. In brief, PLGA (45 mg) and EVG (4 mg) were dissolved in 4 $\mathrm{mL}$ of acetone to obtain PLGA-EVG uniform solution. This PLGA-EVG solution was added dropwise into $10 \mathrm{~mL}$ of $1 \%$ PVA-aqueous solution on a magnetic stir plate at 400 $\mathrm{rpm}$. After 3 hours, $10 \mathrm{mg}$ of PLL was dissolved in $1 \mathrm{~mL}$ water, and $50 \mathrm{mg}$ of pluronic polymer F-68 was dissolved in $4 \mathrm{~mL}$ water. PLL and F-68 solutions were added to the nanoparticle suspension and stirred at room temperature for $\sim 24$ hours to evaporate acetone completely. The non-uniform and larger aggregates of PLGA, PLGA-EVG, PVA, and PLL ingredients were removed by centrifugation at $1,000 \mathrm{rpm}$ for $10 \mathrm{~min}$. The finer and uniform PLGA-EVG NP formulation was stored at $4{ }^{\circ} \mathrm{C}$ or $-20{ }^{\circ} \mathrm{C}$ until further use. A blank PLGA NP formulation was also prepared by using the same protocol without EVG. The hypothetical single NP structure of PLGA-EVG is illustrated in Figure 3-1. PLGA forms the core for holding the native drug EVG and the layers of PVA and PLL were added to ensure better encapsulation efficiency of the EVG inside the NPs. Pluronic F-68 (the PEO and PPO chains of F-68 polymer) was added to maintain a stable formulation for therapeutic application.

\section{Physiochemical Characterization of PLGA-EVG NPs}

The average particle size and zeta potential of the prepared PLGA-EVG nanoparticles were determined by dynamic light scattering (DLS) using a Zetasizer Nano ZS (Malvern Instruments, Malvern, UK). The morphology of PLGA-EVG NPs was confirmed by transmission electron microscope (TEM) (JEOL-2000EX1, Tokyo, Japan). For the DLS measurement, $50 \mu \mathrm{L}$ of freshly prepared $0.5 \mathrm{mg} / \mathrm{mL}$ PLGA-EVG NP was

diluted with distilled water and probe sonicated with ultrasonic homogenizer model 3000 (Biologics, Inc.; Cary NC) for $30 \mathrm{sec}$. Particle size measurements were performed in water for $3 \mathrm{~min}$ at $25^{\circ} \mathrm{C}$. The average of three measurements was recorded. Zeta potential measurement was done in a similar manner, where the NP was diluted with PBS. The zeta potential of NP formulation was also based on the average of 3 readings (each reading $=30$ runs). To study the morphology of NPs, $20 \mu \mathrm{L}$ of the $0.5 \mathrm{mg} / \mathrm{mL}$ of PLGAEVG was carefully placed on the shiny side of the 200 mesh standard TEM grid (Electron Microscopy Sciences, Hatfield, PA). Uranyl acetate solution (2\%; w/v) was used to stain the nanoparticles for achieving positive contrast under TEM. To remove excess amount of formulation, a small piece of filter paper was used, and the grid was allowed to air dry followed by imaging under the TEM system using a direct magnification of 100,000x. To acquire the FTIR spectral data of formulation(s), samples were lyophilized to obtain dry solid powder using a Labconco Freeze Dry System $\left(-48^{\circ} \mathrm{C}\right.$ 133 x 10-3 m Bar; Labconco; Kansas City, MO). FTIR spectra were obtained on the Universal ATR sampling accessory plate using a Spectrum 100 FTIR spectrophotometer 


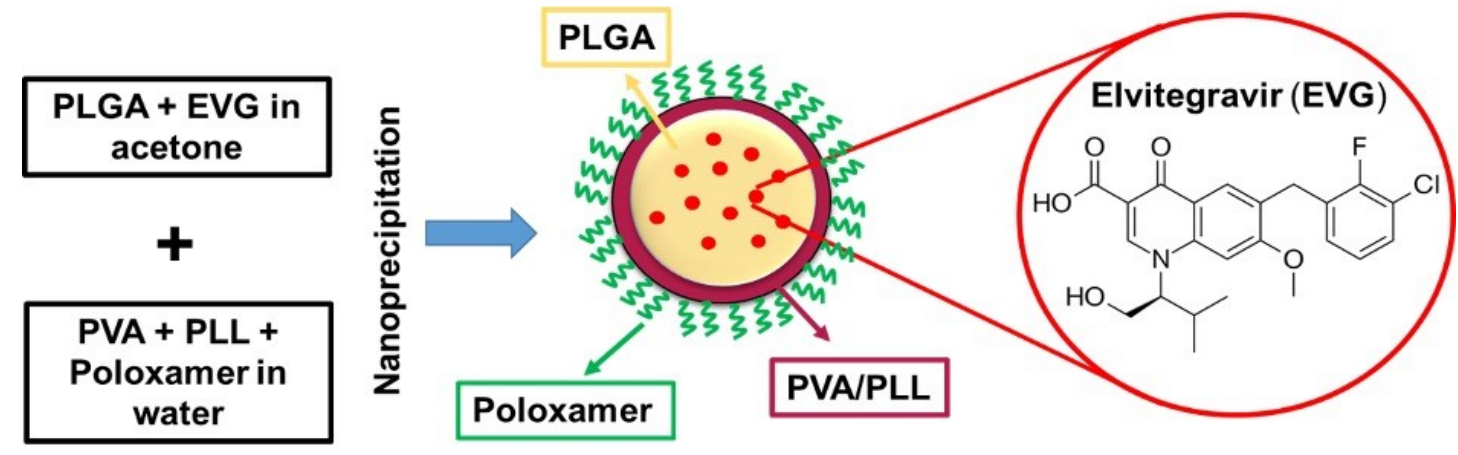

Elvitegravir Nanoparticle Formulation

Figure 3-1. A hypothetical structural representation of the PLGA-EVG NP. 
(Waltham, MA) for drug encapsulation. The samples were placed on the tip of the ATR objective and spectra were obtained between 4000 and $650 \mathrm{~cm}^{-1}$.

\section{In Vitro Drug Release}

The in vitro drug release study was performed using a Float-A-Lyzer ${ }^{\circledR}$ dialysis bag with molecular weight cut-off of 8-10 kDa (Sigma-Aldrich Co. Z726508). A volume of $1 \mathrm{~mL}$ PLGA-EVG NPs was enclosed in a dialysis bag and incubated in $30 \mathrm{~mL}$ cell culture media at $37^{\circ} \mathrm{C}$ under mild agitation in a Thermo Scientific water bath shaker Percision SWB 15 at speed of $35 \mathrm{rpm}$. At 15, $30 \mathrm{~min}$, and 1, 3, 6, 9, 12, $24 \mathrm{~h}, 500 \mu \mathrm{L}$ incubation media was withdrawn and kept at $4{ }^{\circ} \mathrm{C}$ for analyzing of EVG concentration using LC-MS/MS. The same volume of fresh media was added to the incubation media after each sampling.

\section{Cellular Uptake of PLGA NPs in Monocytes}

Cellular uptake of PLGA NPs were assessed by Accuri C6 Flow Cytometer (Accuri Cytometer Inc, Ann Arbor, MI), and images were taken by EVOS ${ }^{\circledR}$ FL Imaging System (AMF4300, Life Technologies; Carlsbad, CA). Monocytes $\left(0.5 \times 10^{6}\right)$ were cultured in a 6-well plate and allowed to attach overnight. For this study, coumarin-6 (C6) dye was encapsulated in PLGA NPs in a similar manner as drug loading in PLGA NPs as PLGA-EVG NPs. The particle size of C6 loaded PLGA was measured by DLS. In this study, C6 dye was used to track, locate, and measure green fluorescence for semiquantitative and qualitative cellular uptake. Cells were treated with $5 \mu \mathrm{g} / \mathrm{mL}$ of PLGA-C6 at different time points $(0.5,1,2,3,4$ hours $)$ and at different concentrations of C6 $(0.5,1$, $2.5,5,10 \mu \mathrm{g} / \mathrm{mL}$ ) for 3 hours. After the course of treatment, cells were washed with PBS, trypsinized, and collected in phenol-red free medium. Then, semi-quantitative and qualitative C6 uptake in PLGA NPs were assessed by using flow cytometry with the FL1 channel (488 excitation, blue laser, $530 \pm 15 \mathrm{~nm}$, FITC/GFP) and EVOS ${ }^{\circ}$ FL Imaging System [83].

\section{Generation of Human Monocytes-Derived Macrophages and Monocytes-Derived Microglia}

Human monocytes-derived macrophages (MDM) and monocytes-derived microglia (MMG) were differentiated from buffy coats that isolated from de-identified human whole blood, which was obtained from Interstate Blood Bank Inc. (Memphis, TN) upon approval from the Institutional Review Board (IRB, UTHSC) according to previously published reports $[84,85]$. Buffy coats were incubated with the RosetteSep enrichment cocktail for 20 min to enrich the yield of peripheral blood mononuclear cells (PBMCs). After 20 min incubation, the buffy coats were diluted with same volume of PBS with $2 \%$ FBS and layered over the lymphocyte separation medium and centrifuged for $20 \mathrm{~min}$ at $1200 \mathrm{~g}$ with acceleration of 1 and deceleration of 0 . A white ring layer 
containing PBMCs formed in between the plasma and lymphocyte separation medium were carefully collected. The collected PBMCs were washed with PBS with 2\% FBS for 3 times to ensure the removal of lymphocyte separation medium. The cells were then incubated with ammonium-chloride-potassium lysing buffer at $4^{\circ} \mathrm{C}$ for 15 minutes to lyse and remove red blood cells. PBMCs for MDM differentiation were collected and cultured in RPMI media containing 10\% human serum, 1\% L-glutamine, and 5\% penicillinstreptomycin. PBMCs for MMG differentiation were collected and cultured in RPMI media containing 1\% L-glutamine and 5\% penicillin-streptomycin. After overnight incubation, non-adherent cells were washed out with PBS. The adherent PBMCs were used for the generation of MDM and MMG. M-CSF (50 ng/mL) was added to the MDM flasks for macrophage differentiation and human recombinant cytokine mixture containing M-CSF (10 ng/mL), GM-CSF (10 ng/mL), NGF- $\beta$ (10 ng/mL), and CCL2 (100 ng/mL) were added to the MMG flasks for microglia differentiation. Mature MDM were collected after 7-10 days differentiation. Mature MMG were collected after 14 days of differentiation when they reached $70-80 \%$ confluence.

\section{HIV-1 Infection of Macrophages and Microglia}

For HIV-1 infection, mature MDM were collected after 7 days of differentiation, followed by incubation with polybrene $(2 \mu \mathrm{g} / \mathrm{mL})$ and HIV-1 Ada strain $\left(30 \mathrm{ng} / 1 \times 10^{6}\right.$ cells) for HIV-1 infection in a 12-well plate with $3 \times 10^{5}$ cells/well. After overnight incubation, MDM were washed twice with PBS to remove the polybrene and HIV-1 Ada strain. The cells attached on the bottom of the well were considered as healthy primary MDM with initial HIV-1 infection. IL-2 $(10 \mathrm{ng} / \mathrm{mL})$ was added to the culture media of MDM to induce HIV-1 infection. Mature MMG were collected after 14 days of differentiation when they reached $70-80 \%$ confluence. One T75 flask of MMG was plated in one 12-well plate with an equal number of cells in each well. MMG were incubated with polybrene $(2 \mu \mathrm{g} / \mathrm{mL})$ and HIV-1 Ada strain at $3 \mathrm{ng} / \mathrm{well}$ for HIV-1 infection. After overnight incubation, MMG were washed twice with PBS to remove the polybrene and HIV-1 Ada strain. After 7-10 days of the initial HIV-1 infection, cell culture media samples from MDM/MMG were collected to assess HIV-1 p24 level using a p24 ELISA kit to confirm the HIV-1 infection.

\section{Intracellular EVG Concentration and Viral Suppression of PLGA-EVG in HIV-1- Infected Macrophages}

Intracellular EVG concentration of PLGA-EVG was measured in HIV-1-infected U937 cells (known as U1 cells) obtained from NIH AIDS Reagent Program. U1 cells were grown in RPMI media supplemented with 1\% L-glutamine, 10\% FBS, and penicillin-streptomycin solution and plated in a 12 -well plate with $3 \times 10^{5}$ cells/well. The cells were differentiated into macrophages using $100 \mathrm{nM}$ phorbol 12-myristate 13-acetate (PMA) for 72 hours. Differentiated cells were treated with EVG native drug at physiological concentration $(0.5 \mu \mathrm{g} / \mathrm{mL})$, and PLGA-EVG NPs $(0.5 \mu \mathrm{g} / \mathrm{mL})$. The EVG 
concentration in cell lysates was determined over a period of $0.1,0.15,0.5,1,3,9,24$, 48, and 72 hours by using LC-MS/MS.

Viral suppression of PLGA-EVG was performed on HIV-1-infected MDM. HIV1 -infected MDM were plated in in a 12 -well plate with $3 \times 10^{5}$ cells/well. MDM were treated with acetonitrile, EVG dissolved in acetonitrile $(0.5 \mu \mathrm{g} / \mathrm{mL})$, PLGA-EVG NPs, $(0.5 \mu \mathrm{g} / \mathrm{mL})$, and PLGA-Blank NPs for 10 days. Equal volume of acetonitrile and PLGABlank NPs were served as control for both EVG native drug and PLGA-EVG NPs. Fresh media was added every three days to ensure cell viability. Cell culture media samples were collected every 24 hours to assess HIV p24 production by p24 ELISA.

\section{EVG Quantification Using LC-MS/MS}

EVG was quantified using the LC-MS/MS method as described in our previous report [86]. The LC-MS/MS system used was a liquid chromatography system (Shimadzu Corp.; Kyoto, Japan) coupled to a tandem mass spectrometer (AB SCIEX, Triple Quad 5500; Framingham, MA). Acquisition was performed in multiple reaction monitoring (MRM) mode, and the Analyst ${ }^{\circledR}$ software (AB Sciex, Foster City, CA) was used for data acquisition and processing. For mass spectrometric analysis, an electrospray ionization (ESI) source operating in positive mode was set with the following parameters: ion spray (IS) voltage: $5500 \mathrm{~V}$; curtain gas: 20 psi; ion source gas 1 (GS1) and ion source gas 2 (GS2): 50 psi; collision-activated dissociation (CAD) gas: 8 psi; source temperature: 400 ${ }^{\circ} \mathrm{C}$. The selected Q1/Q3 (m/z) for EVG was 447.9/343.8 and for internal standard (IS) ritonavir (RTV) is 721.3/296.1. The optimized collision energy (CE) and collision cell exit potential (CXP) for EVG were 43 and $20 \mathrm{~V}$, respectively, with declustering potential (DP) of $100 \mathrm{~V}$ and entrance potential (EP) of $10 \mathrm{~V}$, respectively. The optimized CE and CXP for RTV were 27 and $29 \mathrm{~V}$, respectively, with DP value set at $100 \mathrm{~V}$ and EP value set at $10 \mathrm{~V}$.

EVG standards and experimental samples were extracted by adding either 3 or 9 volumes of cold acetonitrile depend on the sample types, which contained $50 \mathrm{ng} / \mathrm{mL}$ RTV as an internal standard (IS). The mixed solution was vortexed, centrifuged, and analyzed using the LC-MS/MS. In order to reduce matrix effects, calibration curves were prepared with blank media, cell lysate, plasma, or brain homogenate based on the sample types. A $6 \mu \mathrm{L}$ aliquot of extracted samples was injected into an Xterra ${ }^{\circledR}$ MS C18 column $(125 \AA$, $3.5 \mu \mathrm{m}, 4.6 \mathrm{~mm}$ X $50 \mathrm{~mm}$; Waters, Milford) for separation. The mobile phase consisted of (A) water with $0.1 \%$ formic acid and (B) acetonitrile with $0.1 \%$ formic acid (v/v) at a flow rate of $1 \mathrm{~mL} / \mathrm{min}$. The gradient elution was as follows: $0-1.5 \mathrm{~min}, 50 \% \mathrm{~B} ; 1.5-5.1$ $\min , 60 \% \mathrm{~B}(\mathrm{v} / \mathrm{v})$.

\section{Stability Assay}

We determined the particle size and zeta potential of PLGA NPs using DLS. PLGA NPs were stored at room temperature, $4{ }^{\circ} \mathrm{C}$, or $-20^{\circ} \mathrm{C}$ for a stability test. DLS 
analysis was performed on the room temperature sample for 7 days. The size and zeta potential of PLGA NPs from $4{ }^{\circ} \mathrm{C}$ and $-20{ }^{\circ} \mathrm{C}$ were measured on day 7 , and an additional measurement was performed on day 30 for the $-20{ }^{\circ} \mathrm{C}$ sample. The particle size and zeta potential of PLGA NPs in $1 \mathrm{mM}$ HEPES buffer from $\mathrm{pH} 1$ to $\mathrm{pH} 11$ were measured to investigate the alteration of PLGA NPs with this $\mathrm{pH}$ range.

\section{Human Serum Protein Binding to PLGA NPs}

To measure the weight of PLGA NPs, PLGA NP samples were lyophilized to obtain dry solid powder using a Labconco Freeze Dry System. The lyophilized NP samples were weighted and calculated the weight of NPs as per $\mu \mathrm{L}$. Three hundred microgram of PLGA NPs were incubated with $600 \mu \mathrm{L}$ of $1-50 \mathrm{wt} . / \mathrm{v} . \%$ human serum (HS) and stirred at room temperature for 3 hours. The HS-bound PLGA NPs were obtained by a centrifugation of the NPs-HS mixed solution at 10,000 rpm for $20 \mathrm{~min}$. The HS-bound PLGA NPs in the pellet were denoted as \% of HS@NPs. For example, the $10 \%$ HS bound PLGA NPs were recognized as 10HS@NPs. To examine the HS protein adsorption on PLGA NPs, the HS@NPs samples were either resuspended in PBS for a DLS analysis or lyophilized to perform a FTIR spectroscopy.

\section{Human Serum Protein Adsorption of PLGA NPs}

The HS protein adsorption on PLGA NPs was determined by sodium dodecyl sulfate polyacrylamide gel electrophoresis (SDS-PAGE) analysis as described [87]. Briefly, the adsorption capacity of HS proteins was studied based on 3 protocols: 1) equal amount $(300 \mu \mathrm{g})$ of PLGA NPs were incubated with $600 \mu \mathrm{L}$ various concentrations (1$50 \%)$ of HS for a fixed incubation period $(3 \mathrm{~h})$; 2) equal amount $(300 \mu \mathrm{g})$ of PLGA NPs were incubated with $600 \mu \mathrm{L}$ of fixed concentration (10\%) of HS for various incubation periods $(0.5,1,2,3$ and $24 \mathrm{~h})$; and 3$)$ various amount $(10-300 \mu \mathrm{g})$ of PLGA NPs were incubated with $600 \mu \mathrm{L}$ of fixed concentration (10\%) of HS for a fixed incubation period (3 h). After completion of the incubation, the HS@NPs were obtained by centrifugation at 10,000 rpm for $20 \mathrm{~min}$. The supernatant which contained unbound HS proteins were removed. The pellet which contained HS-bound NPs were resuspend in $600 \mu \mathrm{L} \mathrm{PBS}$ and denatured by heating to $95{ }^{\circ} \mathrm{C}$ for 5 min. The HS@NPs samples were separated by size in a $10 \%$ SDS-PAGE analysis using the constant voltage of $150 \mathrm{~V}$ for $75 \mathrm{~min}$. The SDSPAGE gel was processed for a Bio-Safe ${ }^{\text {TM }}$ Coomassie Stain (BioRad; Hercules, CA), and scanned using a BioRad Gel Doc.

\section{Hemocompatibility Assay}

Red blood cells (RBCs) were collected from the de-identified human blood. The freshly collected RBCs were resuspended in RPMI phenol red free media for a hemolysis assay as described in our previous report [87]. Briefly, the RBC suspension $(600 \mu \mathrm{L})$ was incubated with 11-447 $\mu \mathrm{g} / \mathrm{mL}$ EVG or equivalent concentrations of PLGA-EVG, 
30HS@PLGA-EVG, and PLGA-Blank, 30HS@PLGA-blank in Eppendorf tubes for 3 hours at $37^{\circ} \mathrm{C}$. After incubation, the experimental tubes were centrifuged at $1000 \mathrm{rpm}$ for $10 \mathrm{~min}$. The experimental tubes were used for visual inspection and and the supernatant was transferred to a 96-well plate to measure the release of hemoglobin using a plate reader (Cytation 5 imaging, BioTek, Winooski, VT) at $570 \mathrm{~nm}$. Ten microliters of RBCs from the pellet were imaged on a glass slide using an AMF4300 EVOS® FL Imaging System (Life Technologies). RBCs dissolved in MilliQ water was used as positive control \#1 (100\% hemolysis). SDS was used as positive control \#2 (hemo-toxicity), and PBS was used as negative control ( $0 \%$ hemolysis) in this experiment.

\section{Biocompatibility Assay with Macrophages and Microglia}

Cellular toxicity assay was performed on both MDM and MMG in the presence of EVG native drug, PLGA-EVG NPs, and HS30@PLGA-EVG using an XTT cell viability kit (Cell Signaling; Danvers, MA). In this study, MDM and MMG were seeded into 96well plates $\left(2 \times 10^{4}\right.$ cells per well $)$ and grown in a humidified cell culture incubator at 37 ${ }^{\circ} \mathrm{C}$ in a with $5 \% \mathrm{CO}_{2}$. Cells were treated with $0-44.5 \mu \mathrm{g} / \mathrm{mL}$ of EVG or equivalent concentrations of PLGA-EVG, and HS30@PLGA-EVG for 24 hours. The cells were washed twice with PBS and replaced with $100 \mu \mathrm{L}$ fresh media. Subsequently, $50 \mu \mathrm{L}$ of XTT detection solution, which contains electron coupling solution, was added to each well of the 96-well plate. MDM and MMG were incubated with XTT detection solution for 3 hours and absorbance was measured using a plate reader at $450 \mathrm{~nm}$. The percentage of viable cells from treatment groups was calculated by comparing the absorbance with the untreated cells. Data presented are from five replicates. The morphology of MDM and MMG after incubated with different conditions were observed using EVOS ${ }$ FL Imaging System.

\section{Sub-Cellular Localization in Macrophages and Microglia}

In this study, C6, which serves as green fluorescent marker were loaded on PLGA NPs (PLGA-C6 NPs) to visualize the sub-cellular localization of the NPs. Both MDM and MMG were seeded on 4-well cell culture chamber glass slides (CellTreat; Pepperell, MA). Cells were treated with $2.5 \mu \mathrm{g} / \mathrm{mL}$ of PLGA-C6 NPs for 2 hours. Subsequently, cells were incubated with $30 \mathrm{nM}$ MitoTracker ${ }^{\mathrm{TM}}$ Deep Red, $50 \mathrm{nM}$ Texas Red $^{\mathrm{TM}}$ Conjugate, $50 \mathrm{nM}$ CellLight $^{\mathrm{TM}}$ Late Endosomes-RFP, 75 nM LysoTrackerR Red DND99 as markers for mitochondria, early endosome, late endosome, and lysosome, respectively, for 30 minutes. After the incubation, cells were fixed using $4 \%$ paraformaldehyde for 20 minutes and permeabilized with $0.2 \%$ TritonX-100 for 5 minutes. All the cells were stained with DAPI (Life Technologies) and mounted in Vectashield Mounting Medium (Vector Labs, Burlingame, CA) to visualize nuclei. All the images were taken using a laser confocal microscope (Carl Zeiss LSM 710; Oberkochen, Germany) under 400× magnification using an oil immersion objective. 


\section{Cellular Uptake and Internalization Mechanism in Macrophages and Microglia}

MDM and MMG were seeded in 6-well plates at a density of $8 \times 10^{5}$ cells/well. The cells were pretreated with multiple endocytosis inhibitors for $30 \mathrm{~min}$ at $37{ }^{\circ} \mathrm{C}$. The inhibitors used included nocodazole $(10 \mu \mathrm{g} / \mathrm{mL})$, Cyto-D $(10 \mu \mathrm{g} / \mathrm{mL}), \mathrm{CPZ}(10 \mu \mathrm{g} / \mathrm{mL})$, monensin $(200 \mathrm{nM})$, genistein $(200 \mu \mathrm{M})$, and M $\beta C D(1 \mathrm{mM})$ to suppress microtubulerelated endocytosis, macropinocytosis, clathrin-mediated endocytosis, lysosome-involved internalization, caveolae-mediated endocytosis, and caveolae-/clathrin-mediated endocytosis, respectively. The cells were then incubated with $2.5 \mu \mathrm{g} / \mathrm{mL}$ PLGA-C6 NPs for 2 hours at $37^{\circ} \mathrm{C}$. After the treatment, cells were washed twice with PBS, trypsinized, and collected to measure the cellular uptake. Semi-quantitative PLGA-C6 uptake in MDM and MMG were measured using Accuri C6 Flow Cytometer (Accuri Cytometer Inc, Ann Arbor, MI), and images were taken using a laser confocal microscope (Carl Zeiss LSM 710) under 400× magnification using oil immersion objective as described above.

\section{Construction of the In Vitro BBB Model}

The immortalized mouse brain endothelial cells (bEnd.3, CRL-2299) and mouse astrocytes (C8-D1A, CRL-2541) were purchased from American Type Culture Collection. Mouse brain endothelial cells (bEnd.3) and mouse astrocytes (C8-D1A) were cultured in complete media (DMEM media with $10 \%$ fetal bovine serum and $1 \%$ penicillin-streptomycin solution in T75 flasks at $37{ }^{\circ} \mathrm{C}$ in a humidified incubator with $5 \%$ $\mathrm{CO}_{2}$ before building the in vitro BBB model. Transwell ${ }^{\circledR}$-COL collagen-coated $0.4 \mu \mathrm{m}$ pore polytetrafluoroethylene membrane inserts (Sigma-Aldrich) were used to build an in vitro $\mathrm{BBB}$ model as previous published protocols $[88,89]$. Mouse astrocytes were seeded on the bottom of 12 -well plates at a density of $2 \times 10^{4}$ cells/well. After 24 hours of adhesion, mouse endothelial cells were seeded onto the upper side of the Transwell ${ }^{\circledR}$ COL inserts at a density of $2 \times 10^{4}$ cells/well, and the inserts were placed in a 12-well plate containing astrocytes. The in vitro $\mathrm{BBB}$ model including both endothelial cells and astrocytes was grown for 5 days to achieve $\sim 90 \%$ confluency for transmigration assay.

\section{Transendothelial Electrical Resistance Measurements}

The integrity of the tight junction dynamics of the BBB model was measured in terms of transendothelial electrical resistance (TEER) using an EVOM2 Epithelial Voltohmmeter (World Precision Instruments, Sarasota, FL) [90]. The EVOM2 meter was calibrated with a $1000 \mathrm{Ohm}$ test resistor before using. The electrodes from the EVOM2 meter were sterilized by putting in $70 \%$ ethanol for $5 \mathrm{~min}$. Subsequently, the electrodes were preconditioned with the in vitro $\mathrm{BBB}$ cells complete growth media for $5 \mathrm{~min}$. The TEER values were obtained by putting the shorter electrode into the insert and the longer electrode into the bottom chamber of the Transwell ${ }^{\circledR}$ plate to create a current that passed through the membrane. TEER values were measured regularly every 24 hours for 6 days from the day of cell seeding. The TEER values were also measured during the 
experiment period to assess the integrity of the endothelial cell layer. TEER of the blank insert with media alone was subtracted from the TEER final reading to obtain the TEER value for the endothelial monolayer. The TEER value was presented as the value of resistance (current, Ohm) multiplied by the area $\left(\mathrm{cm}^{2}\right)$ of the endothelial monolayer $\left(\mathrm{Ohm} \cdot \mathrm{cm}^{2}\right)$. The electrodes from the EVOM2 meter were cleaned with $1 \%$ Terg-a-zyme ${ }^{\circledR}$ solution to remove the protein or other foreign materials which coated on the electrode after each use.

\section{In Vitro BBB Transmigration Study of PLGA NPs}

The transmigration of PLGA NPs was visualized and measured using fluorescence dye C6 in a confluent in vitro BBB model. PLGA-C6 NPs at concentrations of $2.5,5$, and $10 \mu \mathrm{g} / \mathrm{mL}$ were added to the upper inserts and incubated at $37^{\circ} \mathrm{C}$ for 24 hours. After incubation, astrocytes in the bottom chamber were washed twice with PBS. The media of the bottom chamber was measured using a fluorescence plate reader (SpectraMAX M2e, Molecular Devices; San Jose, CA) with excitation at $488 \mathrm{~nm}$ and emission between 400 and $650 \mathrm{~nm}$. The transmigration of PLGA-C6 NPs was presented in terms of fluorescence intensity. C6 concentration in the media was also calculated using a C6 calibration curve with the matrix of media. The images of recipient astrocytes were taken via EVOS $®$ FL Imaging System.

\section{Penetration of PLGA-EVG NPs in the In Vitro BBB Model}

The in vitro BBB model was used to assess the penetration of the PLGA-EVG NPs. We performed a 24-hour EVG native drug or PLGA-EVG NPs treatment on the BBB cells. EVG native drug/PLGA-EVG NPs at concentrations of 2.5, 5, and $10 \mu \mathrm{g} / \mathrm{mL}$ were added to the upper inserts and incubated at $37^{\circ} \mathrm{C}$ for 24 hours. A sample of $50 \mu \mathrm{L}$ of recipient cell media was collected at 1, 3, 6, 9, and 24-hour time points, and an equal volume of fresh media was added. At the end of the experiment, the cells from the bottom chambers were collected in RIPA buffer and collected. The amount of EVG flux to the bottom chambers from both media and cell lysate was measured using LC-MS/MS method.

\section{Mechanistic Contribution of P-gp in Opposing the Penetration of EVG Across the In Vitro BBB Model}

The mechanistic contribution of P-gp in interfering with the penetration of EVG was assessed in the presence and absence of the P-gp inhibitor elacridar. The in vitro BBB layer were pre-incubated with or without elacridar $(500 \mathrm{nM})$ for $30 \mathrm{~min}$ before a penetration study. After pre-incubation, $5 \mu \mathrm{g} / \mathrm{mL}$ of EVG native drug or PLGA EVG NPs were added to the upper inserts and incubated at $37^{\circ} \mathrm{C}$ for 24 hours. A sample of $100 \mu \mathrm{L}$ of media from the bottom chamber was collected at 1,3,6,9, and 24-hour time point, and 
an equal volume of fresh media was added. The amount of EVG flux to the bottom chambers was measured using LC-MS/MS.

\section{EVG Levels in the In Vivo Mouse Model}

Male NOD.Cg-Prkdc ${ }^{\text {scid }} I l 2 \mathrm{rg}^{\mathrm{tm} / \mathrm{Wjl}} / \mathrm{SzJ}$ (NSG) mice were purchased from Jackson Laboratory (Bar Harbor, ME) and allowed to acclimate in the animal facility for at least 7 days. A total of 16 mice were divided into two groups: one group of 8 mice was injected intraperitoneally (i.p.) with $20 \mathrm{mg} / \mathrm{kg}$ EVG native drug in 0.1-0.2 mL of PBS. Another group of 8 mice was injected i.p. with $20 \mathrm{mg} / \mathrm{kg}$ of PLGA-EVG NP in $0.1-0.2 \mathrm{~mL}$ of PBS. Mouse blood was collected at 3, 9, 24, 48, 72, and 96 hour timepoints in EDTAcontaining blood collection tubes following a serial tail sampling procedure [91]. Briefly, for each mouse, mouse was placed in a mouse restrainer and mouse tail was pre-warmed by putting the tail at $37^{\circ} \mathrm{C}$ of water for $2 \mathrm{~min}$. A $25 \mathrm{G}$ needle was used to stick the tail and $\sim 20-50 \mu \mathrm{L}$ blood was quickly collected using a pipette. For each mouse, the first time point was collected at the distal end of the tail, and each subsequent time point was collected a few millimeters up from the previous stick towards the base of the tail. After collection, the blood was quickly diluted by equal volume of PBS. Mouse blood was settled down at room temperature and then centrifuged at 6,000 rpm for $10 \mathrm{~min}$ for plasma harvesting. After blood collection, the mice were sacrificed by exsanguination under deep isoflurane anesthesia, followed by cervical dislocation at 96 hours. Then, the brain tissues were harvested. Mice plasma and brain tissues were placed into tubes and frozen at $-80{ }^{\circ} \mathrm{C}$ until further analysis by LC-MS/MS. All experimental protocols involving the use of laboratory animals were approved by the UTHSC Institutional Animal Care and Use Committee (IACUC).

\section{Viral Suppression of PLGA-EVG NPs in HIV-1-Infected Macrophages and Microglia after Crossing the In Vitro BBB Model}

To determine the efficacy of the viral suppression of PLGA-EVG NPs, we used $\mathrm{HIV}$-1-infected primary MDM and MMG to create a modified in vitro BBB model in a Transwell ${ }^{\circledR}$ plate. The confluent brain endothelial monolayer was obtained as described in the previous section. The upper insert of the in vitro BBB model containing confluent brain endothelial cells was transferred to the 12-well plate containing HIV-1-infected MDM or MMG, and co-cultured with the infected cells. In this modified in vitro BBB model, HIV-1-infected MDM/MMG were cultured on the bottom of the 12-well plate. Endothelial monolayers in the upper inserts were exposed to control, blank PLGA NPs, EVG native drug $(5 \mu \mathrm{g} / \mathrm{mL})$, and PLGA-EVG NPs $(5 \mu \mathrm{g} / \mathrm{mL})$ for 7 days. HIV-1 viral loads from the culture media of the bottom chamber were measured every day using a p24 ELISA kit. The upper inserts were replaced with fresh BBB cells after 3 days to keep BBB monolayer confluent. A mean TEER value of 100 to $120 \mathrm{Ohm} \cdot \mathrm{cm}^{2}$ was obtained every day and was consistent with the modified BBB model during the time of the experiment. All the HIV-1- related experiments were performed in the BSL-3 laboratory in the Regional Biocontainment Laboratory of UTHSC. 


\section{Viral Suppression of PLGA-EVG NPs in HIV-1 Encephalitic (HIVE) Mice}

Four-week-old male NSG mice were purchased from The Jackson Laboratory (Bar Harbor, ME). All experimental protocols involving the use of laboratory animals were approved by the IACUC of the University of Nebraska Medical Center and the NIH. To create the HIV-1-infected mouse model, NSG mice were administered i.p. with anesthesia ketamine/xylazine (100 mg/kg ketamine and $16 \mathrm{mg} / \mathrm{kg}$ xylazine), NSG mice were injected bilaterally into the basal ganglia with $5 \times 10^{5}$ cell suspension in $5 \mu \mathrm{L}$ containing HIV-1 ADA-infected MDM (total $n=6$ ). Fourteen days after the MDM injection, the animals were injected i.p. with vehicle control (PLGA-Blank NPs), EVG native drug, or PLGA-EVG NPs in 0.1-0.2 mL of 5\% dextrose divided in three times on the first day to reach the dose of $200 \mathrm{mg} / \mathrm{kg}$ ( $\mathrm{n}=2$ in each group). Mice were sacrificed on day 5 after the drug injection. Brain samples were collected and used for RNA extractions, and CNS HIV-1 p24 levels were assessed by measuring HIV-1 gag RNA levels using RT-PCR. GAPDH was used as a housekeeping gene in this RT-PCR analysis.

\section{Statistical Analysis}

All graphs and statistical analyses were performed using GraphPad Prism 5 (GraphPad Software; La Jolla, CA). The statistical significance between two groups was determined by student's t-test analysis. The statistical significance among treatment groups with individual variable was determined by one-way ANOVA Tukey HSD posthoc test. The statistical significance among treatments groups with two variables were determined by using two-way ANOVA with a Bonferroni post-hoc test. A p-value $\leq 0.05$ was considered to be significant. 


\section{CHAPTER 4. RESULTS AND DISCUSSION ${ }^{1}$}

\section{Novel EVG Nanoformulation Approach to Suppress the Viral Load in HIV-1- Infected Macrophages}

Monocyte/macrophage lineage cells express the CD4 receptor and chemokine coreceptors for entry of HIV-1 [1]. Thus, they perform an important role in initial HIV-1 infection. Monocytes and macrophages are resistant to HIV-induced apoptosis and are a crucial reservoir for the virus $[92,93]$. ARVs has notably improved the life of HIV-1 infected patients. However, it is difficult to maintain therapeutic levels of ARVs in monocytes and macrophages because of drug efflux transporters and drug metabolic enzyme cytochrome, such as MRP1 and CYP3A4, respectively, are expressed in these cells [94-96]. INSTIs showed a higher antiviral activity in PBMCs and macrophages than lymphocytic T cells, while PIs show a 2- to 10-fold lower efficacy in monocytes than in T-lymphocytes $[97,98]$. Thus, a new strategy to improve the bioavailability and efficacy of the ARVs in monocytes and macrophages may be achieved by INSTIs.

Most ARVs are the substrates of efflux transporters and drug metabolic enzymes, requiring combination therapy and high doses for treatment [25]. HIV can also potentially develop resistance to treatment with these drugs $[99,100]$. EVG, one of the most prescribed and the newest class of ARVs, is also a substrate of CYP3A4 [101]. Thus, in many regimens EVG is formulated with cobicistat, a CYP3A4 inhibitor, as a pharmacological enhancer $[86,102]$. Therefore, it is desirable to develop a NP-based formulation for EVG that is likely to bypasses drug transporters and metabolic enzyme in the liver as well as in HIV-1-infected monocytic cells. The main objective of this chapter is to develop an EVG nanoformulation that shows increased efficacy in monocytic cells.

\section{Physiochemical Characterization of PLGA-EVG NPs}

To ensure that there is no significant difference in particle size of PLGA NPs when introduced to in vitro conditions, we measured the particle size both in water and cell culture media. Before drug loading, blank PLGA NPs were measured for particle size by DLS, and demonstrated a size of $80 \pm 4.43 \mathrm{~nm}$ and $89 \pm 4.13 \mathrm{~nm}$ in water and cell culture media, respectively. To further determine if drug loading to these particles causes

${ }^{1}$ Portions of chapter from previously published article; final submission modified with permission. Gong, Y., et al. (2017). Novel elvitegravir nanoformulation approach to suppress the viral load in HIV-infected macrophages. Biochemistry and Biophysics Reports, 2017. 12: p. 214-219. DOI: https://doi.org/10.1016/j.bbrep.2017.10.005 [81]. Portions of chapter reprinted from final submission with open access permission. Gong, Y., et al. (2020) Novel elvitegravir nanoformulation for drug delivery across the bloodbrain barrier to achieve HIV-1 suppression in the CNS macrophages. Sci Rep 2020. 10, 3835. DOI: https://doi.org/10.1038/s41598-020-60684-1 [82]. 
any difference in size, we conducted DLS particle size measurement of the EVG loaded PLGA NPs. Particle size distribution histograms confirm that there is a slight increase in the size of drug loaded particles over blank NPs, with EVG loaded PLGA NPs size of $105 \pm 1.79 \mathrm{~nm}$ in water and $109 \pm 4.10 \mathrm{~nm}$ in cell culture media (Figure 4-1A).

The slight increase in size is considered low enough for active targeting to highly replicating HIV-1-infected cells. The zeta potential of fabricated NPs was measured using DLS (Figure 4-1B). DLS measurements confirms that the NP has a low zeta potential of -6.74 mV. PLGA-EVG NPs under TEM (Figure 4-1C) suggesting that PLGA-EVG NPs have a spherical morphology and are $40-50 \mathrm{~nm}$ particle size. TEM measurement provided the particle size of PLGA NPs in a dried form while DLS gave the particle size in suspension which provided a hydrodynamic diameter that included NPs and any molecule attached or adsorbed on the surface. Thus, the particle size obtained from TEM measurement exhibited a smaller size compared with the size obtained from DLS measurement. The obtained particle size range and low positive zeta potential of PLGAEVG NPs are recommended and suitable for effective drug delivery purposes [103]. The FTIR analysis of EVG, PLGA-EVG, and blank PLGA NPs is shown in Figure 4-1D. A broad peak between $3600-3050 \mathrm{~cm}^{-1}$ was found in PLGA-EVG and blank PLGA NPs due to the presence of hydroxyl groups of the PLGA/F68 polymer $[104,105]$. The characteristic peak of EVG was observed in the PLGA-EVG spectrum within specified range. The FTIR spectral analysis indicated that EVG was encapsulated in NPs. The in vitro release of PLGA-EVG NPs in cell culture media is shown in Figure 4-1E. The release of EVG from PLGA NPs was followed by a zero-order release profile with $\mathrm{r}^{2}$ of 0.98 in $24 \mathrm{~h}$. Only $\sim 15 \%$ of cumulative EVG release was observed at $24 \mathrm{~h}$, which suggested the potential controlled release profile of PLGA-EVG NPs. The drug release from PLGA NPs may relate to the loading ratio of drug with the PLGA copolymer, NP protected layer stability, and/or physicochemical properties of EVG.

PLGA is an FDA-approved biodegradable polymer with minimal systemic toxicity used for drug delivery [106]. PLGA has been extensively used in the formulation of many anticancer drugs [83, 107-110]. Recently, PLGA has also been used to formulate EVG and tenofovir alafenamide using emulsion solvent evaporation method for longacting prevention of HIV-1 vaginal transmission [70]. The PLGA NP formation in our study is an oil-in-water $(\mathrm{o} / \mathrm{w})$ emulsification, which is an ideal method for waterinsoluble drugs [111]. PLGA is the main polymer core to hold EVG molecules, PVA is used to stabilize the emulsion, poloxamer 188 (Pluronic polymer F68) is used as a stabilizer and to reverse drug resistance, and PLL is used to provide slight positive charge, which helps internalization into the target cells [106, 112, 113]. According to our results, PLGA-based EVG formulation is within the size range of $50 \mathrm{~nm}$, so it is easily diffused through cell membrane to deliver the drugs.

\section{Cellular Uptake of PLGA NPs}

The cellular uptake study was performed using the fluorescence dye C6. To ensure that the C6 loaded PLGA NPs were of similar size to the parent moiety, we 
A

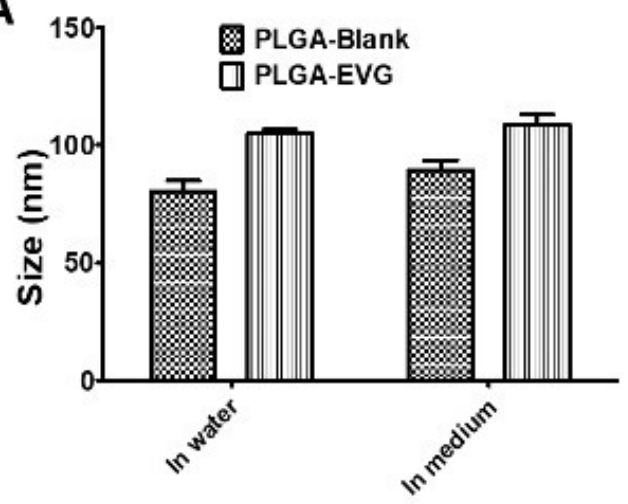

C

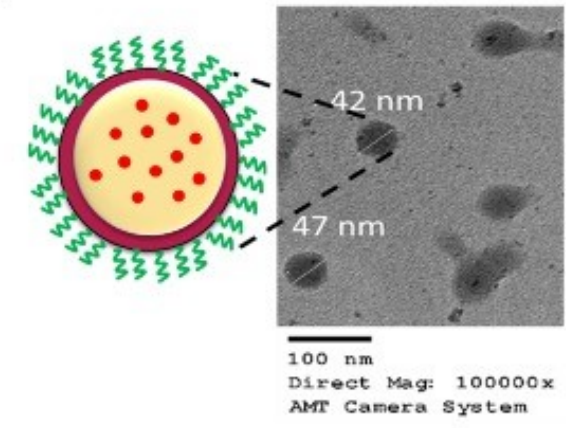

B

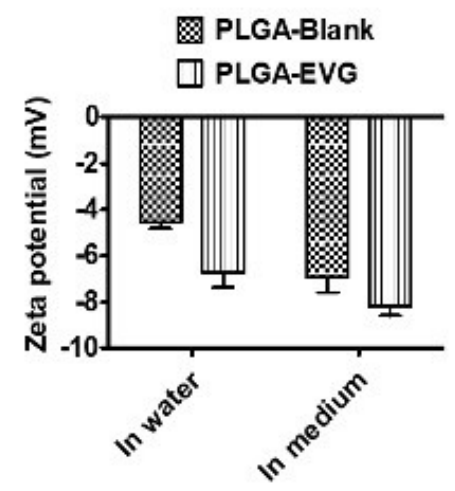

D

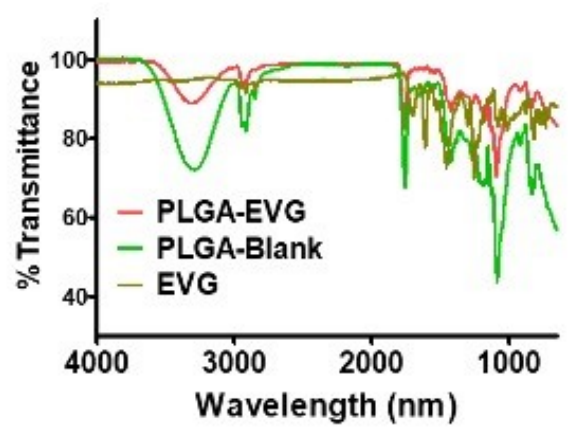

E

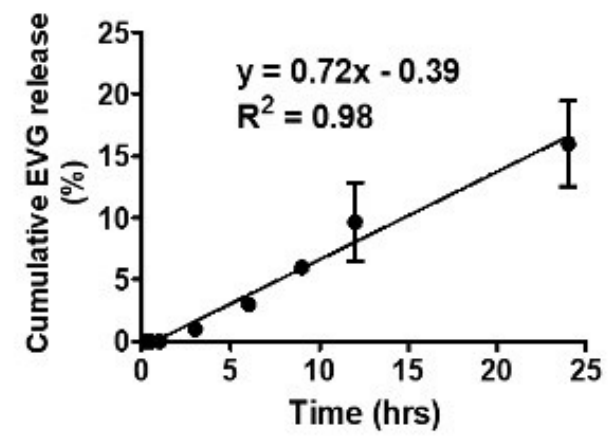

Figure 4-1. Physiochemical characterization of PLGA-EVG NPs.

(A) DLS-based particle size distribution. (B) Zeta potential of PLGA-EVG NPs. (C) Morphology of PLGA-EVG NPs as viewed under TEM by using AMT camera at a direct magnification of $100,000 \mathrm{x}$. Bar equals to $100 \mathrm{~nm}$ in the TEM image. (D) FT-IR spectral confirmation for drug encapsulation. (E) In vitro release profile of PLGA-EVG NPs. Mean \pm SEM values were graphed from 3 independent experiments. * indicates $\mathrm{p}<$ 0.05 . 
measured the particle size by DLS prior to this study. PLGA-C6 NPs were $85 \pm 2.83 \mathrm{~nm}$ and $93 \pm 2.90 \mathrm{~nm}$ in water and medium, respectively. The cellular uptake of PLGA NPs in monocytes was presented in terms of mean fluorescence intensity.

A time-dependent increase in fluorescence intensity was observed (Figure 4-2A-B), with the highest fluorescence after 4 hours of exposure. Similarly, an increase in fluorescence intensity was observed with increasing concentrations of C6 (Figure 4-2C-D). The highest fluorescence was observed in the cells treated with $10 \mu \mathrm{g} / \mathrm{mL} \mathrm{C6}$. Overall, the results showed that cellular uptake of PLGA NPs followed time- and concentration-dependent profiles.

\section{Intracellular EVG Concentration and Viral Suppression Efficacy of PLGA-EVG NPs in Macrophages}

To evaluate the intracellular concentration of EVG, we performed LCMS-MS using RTV as an internal control. EVG and RTV are eluted separately at approximately 3.27 and $2.72 \mathrm{~min}$, respectively (Figure. 4-3A). The standard curve was achieved over the range of $1-500 \mathrm{ng} / \mathrm{mL}$ using linear regression $\left(\mathrm{r}^{2}=0.99\right)$ with a weighting factor of $1 / \mathrm{x}^{2}$. We performed a short-term EVG/PLGA-EVG treatment on U1 cells. The time profiles of EVG and PLGA-EVG as the percentage of initial EVG concentration up to 72 hours are shown in Figure 4-3B. The results showed an overall increased EVG concentration in PLGA-EVG NP treatment compared to EVG alone. When compared each measured time point, PLGA-EVG NP showed a significance increase in EVG concentration compared with EVG alone. Furthermore, we calculated $\mathrm{AUC}_{(0-\mathrm{t})}$ for EVG from both PLGA-EVG and EVG alone. AUC $(0-$ t) of PLGA-EVG showed a significant ( 2-fold) increase compared to EVG alone (Figure 4-3C, ${ }^{*} p<0.5$ ). These results demonstrate that the PLGA-EVG NPs improve the intracellular delivery and drug retention in U1 cells

To determine the efficacy of the viral suppression of PLGA-EVG NPs, HIV-1infected primary macrophages were exposed to vehicle control, blank PLGA NPs, EVG, or PLGA-EVG NPs for 9 days. HIV-1 p24 protein levels were measured every 24 hours, and the results are shown in Figure 4-4A. PLGA-Blank NPs showed no effect on viral replication. When compared the overall effect with control cells, both EVG and PLGA EVG NPs showed a decreased viral load. When compared the treatment efficacy on each measurement days, cells treated with PLGA EVG NPs showed a decreased viral load compared with control cells from day 2 to day 5, and a better antiviral efficacy on day 4 and day 5 compared with EVG treated cells.

Since HIV-1 replicates within the cells, intracellular drug concentrations are important to determine the efficacy of ARVs [94]. The intracellular concentrations of ARVs, especially PIs and INSTIs, are dependent on the drug metabolizing enzyme CYP3A4, and on efflux transporters, such as MRP1 and P-gp [86, 96, 114, 115]. These transporters and metabolic enzymes are highly abundant in the gut and liver, and is the main cause for developing resistance [116]. To enhance the bioavailability of these 


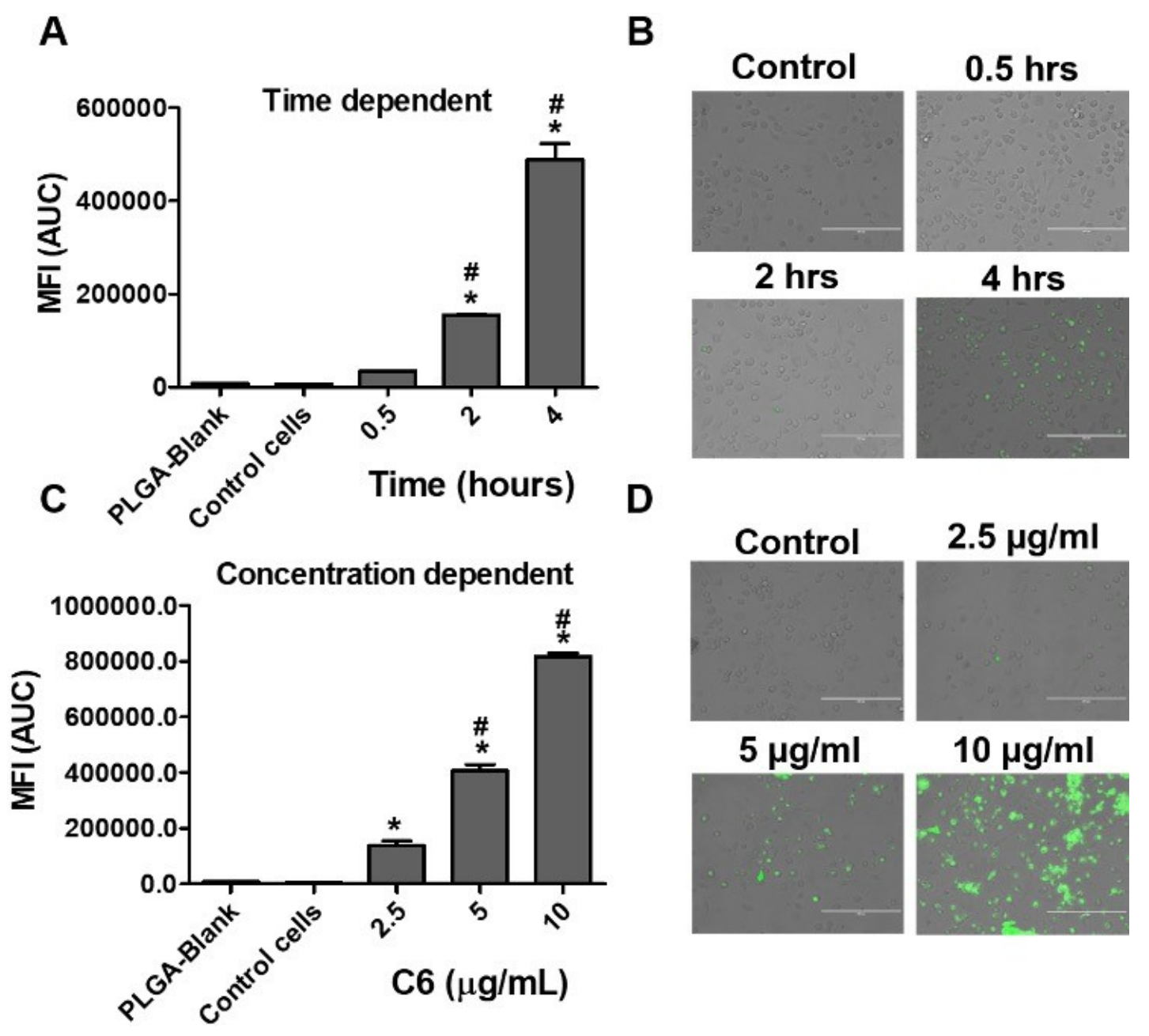

Figure 4-2. Cellular uptake of nanoparticles.

(A-B) Time-dependent cellular uptake of PLGA NPs in monocytic cells. (C-D)

Concentration-dependent uptake of NPs in monocytic cells. Qualitative uptake was confirmed by EVOS ${ }^{\circledR}$ FL Imaging System and semi-quantitative uptake was determined by Accuri C6 Flow Cytometer. Bar equals to $200 \mu \mathrm{m}$ in microscopic images B, D. * indicates $\mathrm{p}<0.05$ compared with PLGA-Blank, \# indicates $\mathrm{p}<0.05$ compared with the previous time point/concentration. 
A

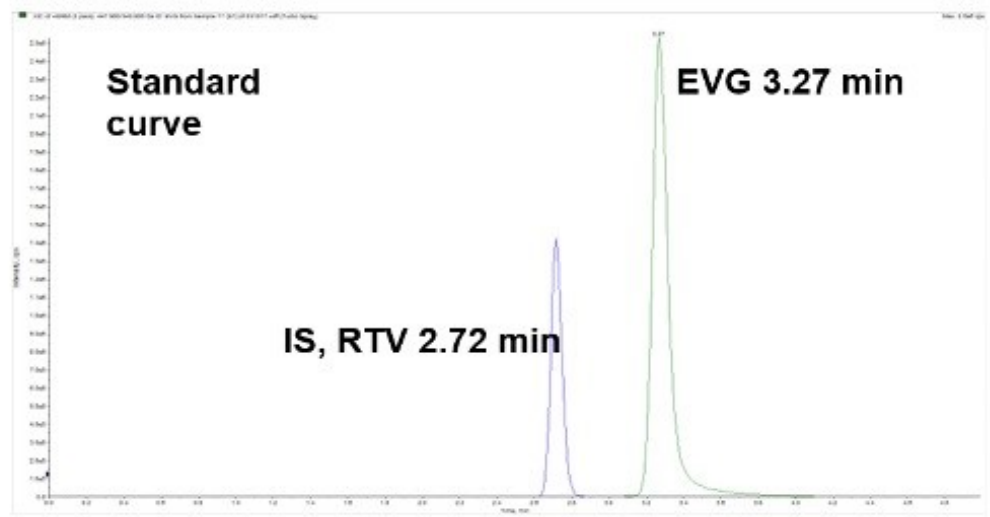

B

C
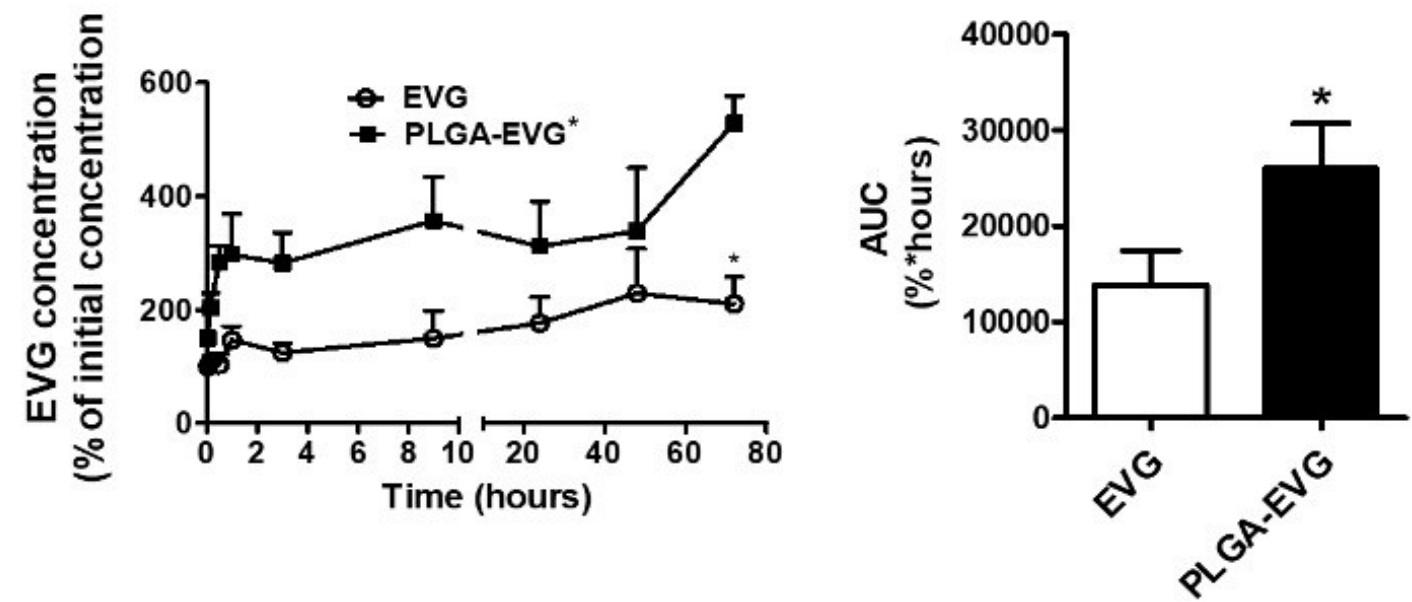

Figure 4-3. Intracellular EVG concentration in U1 cells.

(A) LC/MS chromatogram peaks of EVG and its corresponding internal standard (IS), RTV as well as standard curve for EVG measurement. (B) Concentration time profile of EVG and PLGA-EVG NPs in U1 cells. (C) Area under the curve (AUC) of EVG and PLGA-EVG NPs. Mean \pm SEM values were graphed from 3 independent experiments. *indicates $\mathrm{p}<0.05$ compared to $\mathrm{EVG}$ alone treatment. 
A
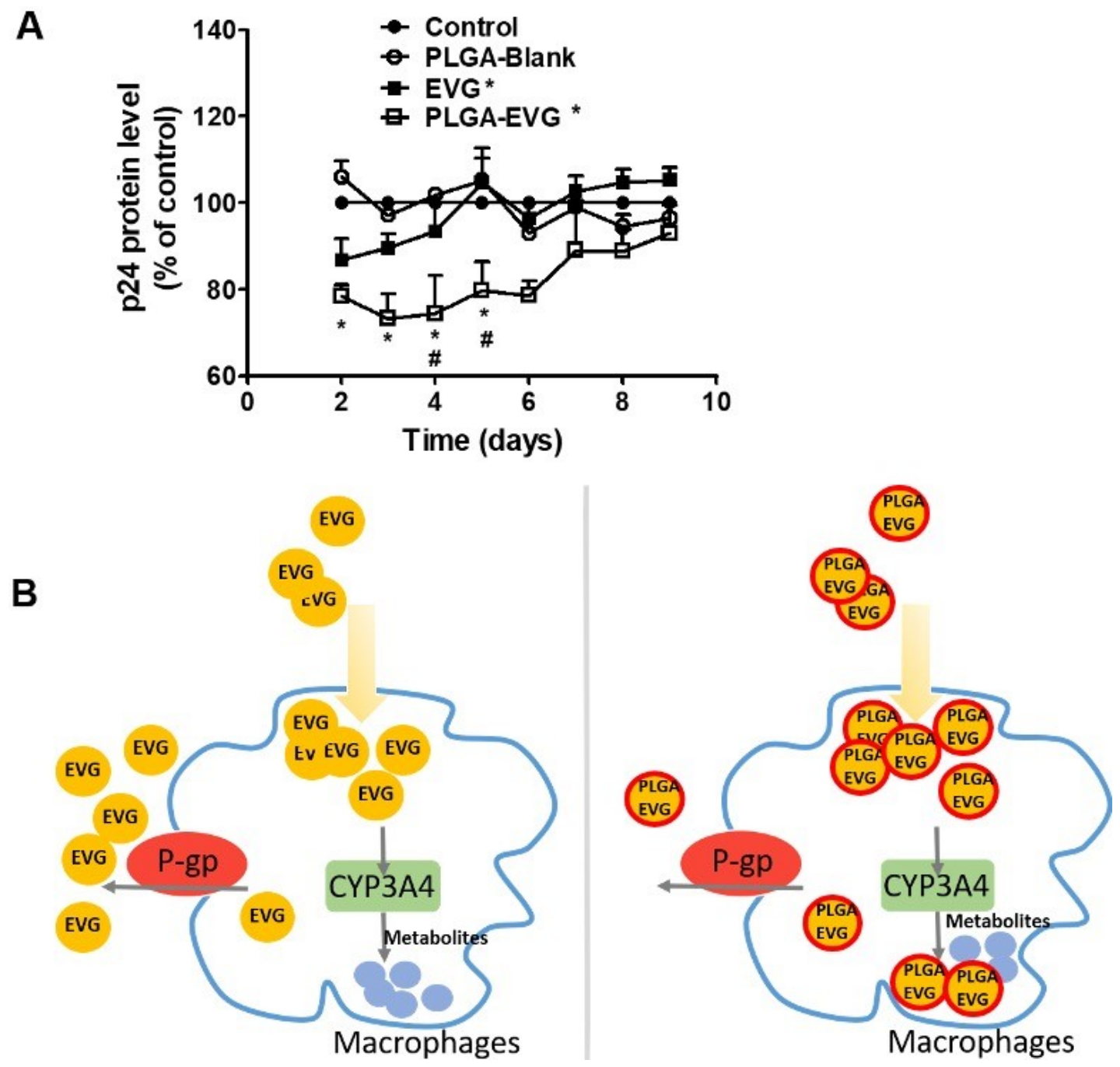

Figure 4-4. Viral suppression of PLGA-EVG NPs.

(A) Time course of HIV replication upon drug exposure in HIV-1-infected MDM. Mean \pm SEM values were graphed from 3 independent experiments. (B) A hypothetical mechanism that may explain the higher EVG concentration in PLGA-EVG NPs than EVG alone. *indicate $\mathrm{p}<0.05$ compared to control; \#indicate $\mathrm{p}<0.05$ compared to EVG alone treatment. Mean \pm SEM values were graphed from 3 independent experiments. 
ARVs, potent inhibitors of CYP3A4 such as cobi are generally used. We have recently shown the abundance of drug transporters and CYP3A4 in monocytic cells $[95,96]$. Thus, bypassing transporters and CYP3A4 is considered as an effective strategy to improve the ARV intracellular concentrations, especially in monocytic cells. Literature reports suggest that NPs-based drug delivery would bypass the efflux transporters such as P-gp [117-119], as well as liver metabolism [120]. The hypothetical mechanism behind the difference in intracellular concentrations of EVG and PLGA-EVG in macrophages is shown in Figure 4-4B. Based on our findings, we hypothesize that higher concentration of EVG in PLGA-EVG treatment than EVG alone results from its likely protection from drug transporter P-gp and metabolic enzyme CYP3A4. Our hypothesis is further strengthened from the literature data that the concentration of ARVs is relatively lower in P-gp- and CYP3A4-expressing monocytic cells than in lymphocytic cells [2, 5-7]. Moreover, co-administration of EVG with cobicistat is relatively expensive, and is not affordable to African and Asian countries where HIV-1 prevalence is the highest. The use of multiple drugs are also likely to cause drug-drug interactions, leading to toxicity in individuals who also take other drugs that are the substrates, inducers, or inhibitors of CYP3A4 [121, 122]. Thus, developing an optimal EVG formulation without cobicistat can minimize these drug-drug interactions. Compared to EVG alone, the PLGA-EVG NPs can provide a high intracellular drug concentration without need for a pharmacoenhancer, and present relatively greater viral suppression in primary macrophages. Overall, this formulation is a step forward to develop EVG nanomedicine that suppresses viral replication at a relatively low dose. Because of the prolonged viral suppression time, this nanoformulation is also likely to reduce the probability for developing drug resistance.

\section{Stability, Protein Corona, Biocompatibility, and Cellular Internalization Pathway of EVG Nanoformulation}

In the previous section, we developed a PLGA-based nanoformulation containing EVG. Our PLGA-EVG NPs showed improved intracellular uptake of EVG in macrophage cell line and viral suppression in HIV-1-infected primary macrophages. As part of the recommended initial regimen for the majority of HIV-1-infected populations in the USA [68], researchers started formulating EVG into NPs to combat HIV-1 infection in the last three years $[69,70]$. For example, EVG has been formulated into PLA NPs with a HPG coating on the surface [69]. These PLA NPs were distributed widely throughout the reproductive tract, and showed a prolonged intravaginal delivery of EVG. Moreover, EVG has been used together with tenofovir alafenamide in a PLGAbased NPs coated with poloxamer 407 as a prevention strategy to against HIV-1 [70]. These EVG NPs can extend the half-life $t_{1 / 2}$ of EVG from 10.8 hours to 3.3 days and protect mice with $100 \%$ and $60 \%$ uninfected rate on day 4 and day 14 post-nanoparticle injection after a HIV-1 challenge. Although these studies have demonstrated the use of nanocarriers to deliver EVG, none of these strategies have moved to clinical research yet. One of the reasons is the limited understanding of the fate and biosafety of NPs in the physiological system [87]. For example, it is expected that human serum protein binding occurs when NPs are administer to human subjects [123]. Upon introduction of NPs into 
the body, NPs dynamically interact with serum proteins and form the NP-protein complexes [124]. Understanding the interaction of NPs with HS can predict their safety and efficacy profiles when used in the clinic [123].

In addition, determination of hemocompatibility with red blood cells (RBCs) is important for a formulation which is intended for systemic administration, as an incompatible formulation may lead to a thromboembolism which can cause the death of the patient [125]. Therefore, the aim of this chapter is to identify the interaction of HS proteins and PLGA NPs, and the subsequent effect on hemocompatibility with RBCs and biocompatibility with macrophages and microglia. In addition, we also identified the internalization mechanism of the PLGA NPs in macrophages and microglia to further understand the interaction of PLGA NPs with HIV-1 reservoirs macrophages and microglia in the CNS.

\section{Stability of PLGA NPs}

First, a stability study was performed for the PLGA NP formulation to establish expectations for the storage shelf life of the prepared formulation. The size of freshly made PLGA NPs was approximately $135.7 \pm 1.5 \mathrm{~nm}$ in PBS (Table 4-1). PLGA NPs were stable at room temperature for at least a week. No specific change in particle size or zeta potential of NPs was observed for 7 days when stored at room temperature. PLGA NPs can be stored at $4{ }^{\circ} \mathrm{C}$ and $-20{ }^{\circ} \mathrm{C}$ for at least 30 days with no change in particle size or zeta potential. PLGA NPs showed a stable particle size in $1 \mathrm{mM}$ HEPES buffer within the $\mathrm{pH}$ range of 1-11 (Table 4-2). PLGA NPs showed a positive charge $(\sim 7 \mathrm{mV})$ at all the recorded $\mathrm{pH}$ values except $\mathrm{pH} 4,5$, and 11. A higher positive charge $(\sim 20 \mathrm{mV})$ of PLGA NPs was observed at the $\mathrm{pH}$ range of $4-5$, and a lower charge $(\sim 3 \mathrm{mV})$ of PLGA NPs was observed at $\mathrm{pH} 11$

\section{Evaluation of Human Serum Protein Binding on PLGA NPs}

We showed the existence of an HS protein corona on PLGA NPs using DLS, FTIR, and SDS-PAGE analyses. A DLS assay of PLGA NPs was investigated in PBS and $1-50 \% \mathrm{HS}$. An increase in particle size to $200 \mathrm{~nm}$ was observed for PLGA NPs in $1-50 \%$ HS (Figure 4-5A). The reason of the increased particle size is due to the binding of HS protein onto PLGA, which establishes a soft corona[87]. HS proteins are loosely bound to the PLGA NPs, leading to this increase in particle size. The increased size of PLGA NPs is acceptable because NPs with size of $200 \mathrm{~nm}$ can be taken up by human brain endothelial cells efficiently [126]. Previous study also showed that NPs with size of $200 \mathrm{~nm}$ also provided a compatible BBB transportation compared with NPs with size of $100 \mathrm{~nm}$ [127]. For polymeric NPs, the size between $60 \mathrm{~nm}$ to $200 \mathrm{~nm}$ still considered to have a very large surface-to-volume ratio to show the optimal drug uptake. Zeta potential of PLGA NPs was observed to be negative in 1-50\% HS (Figure 4-5B). This negative charge is expected, because HS is negatively charged in $\mathrm{pH} 7.4$ PBS. The negative zeta

potential indicates that PLGA NPs are stable and safe for therapeutic applications. PLGA 
Table 4-1. Size and zeta potential of PLGA NPs stored at room temperature, $4^{\circ} \mathrm{C}$ and $-20{ }^{\circ} \mathrm{C}$.

\begin{tabular}{|c|c|c|c|c|c|c|c|c|c|c|c|c|}
\hline \multirow[b]{2}{*}{ Days } & \multicolumn{8}{|c|}{ Room temperature } & \multicolumn{2}{|c|}{$4^{\circ} \mathrm{C}$} & \multicolumn{2}{|c|}{$-20{ }^{\circ} \mathrm{C}$} \\
\hline & Day 0 & Day 1 & Day 2 & Day 3 & Day 4 & Day 5 & Day 6 & Day 7 & Day 7 & Day 30 & Day 7 & Day 30 \\
\hline Size (nm) & 135.7 & 134.4 & 135.5 & 135.7 & 132.6 & 134.2 & 135.9 & 134.6 & 132.1 & 133.1 & 131.9 & 132.9 \\
\hline & \pm 1.5 & \pm 0.7 & \pm 0.5 & \pm 0.4 & \pm 0.6 & \pm 1.3 & \pm 1.4 & \pm 1.2 & \pm 0.5 & \pm 0.5 & \pm 0.2 & \pm 0.3 \\
\hline Zeta potential & 0.11 & -0.53 & -0.18 & -0.02 & 0.62 & -0.37 & 0.00 & -0.18 & -0.25 & -0.22 & -0.02 & -0.12 \\
\hline$(\mathrm{mV})$ & \pm 0.5 & \pm 0.3 & \pm 0.3 & \pm 0.2 & \pm 0.2 & \pm 0.2 & \pm 0.2 & \pm 0.3 & \pm 0.2 & \pm 0.3 & \pm 0.3 & \pm 0.3 \\
\hline
\end{tabular}

Mean \pm SEM values were calculated from three measurements. Size and zeta potential of NPs from different storage conditions were compared with day 0 , room temperature sample.

Table 4-2. Size and zeta potential of PLGA NPs in 1mM HEPES buffer from pH 1 to pH 11.

\begin{tabular}{|c|c|c|c|c|c|c|c|c|c|}
\hline pH range & pH 1 & pH 3 & pH 4 & pH 5 & pH 6 & pH 7.4 & pH 8 & pH 10 & pH 11 \\
\hline \multirow[t]{2}{*}{ Size (nm) } & 132.2 & 132.5 & 134.4 & 133.8 & 132.1 & 132.0 & 131.5 & 126.9 & 128.3 \\
\hline & \pm 1.0 & \pm 0.8 & \pm 0.8 & \pm 0.9 & \pm 0.4 & \pm 0.4 & \pm 1.2 & \pm 0.7 & \pm 1.5 \\
\hline Zeta potential & 7.02 & 6.36 & 18.87 & 22.27 & 7.72 & 7.94 & 6.90 & 5.87 & 3.40 \\
\hline$(\mathrm{mV})$ & \pm 0.2 & \pm 0.6 & \pm 1.8 & \pm 0.4 & \pm 1.3 & \pm 0.75 & \pm 0.55 & \pm 0.34 & \pm 0.31 \\
\hline
\end{tabular}

Mean \pm SEM values were calculated from three measurements. 
A

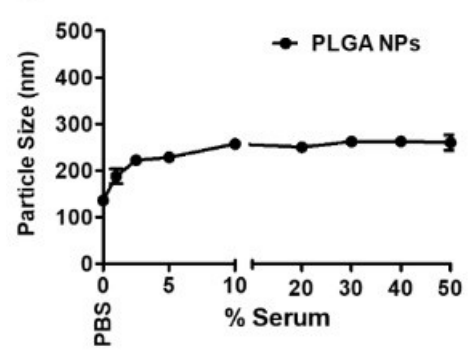

B

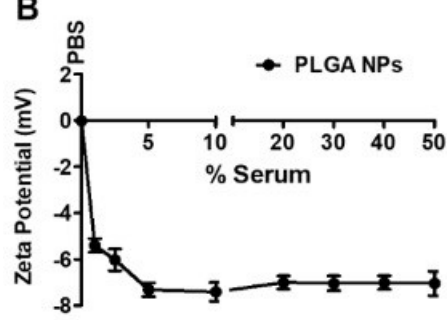

C
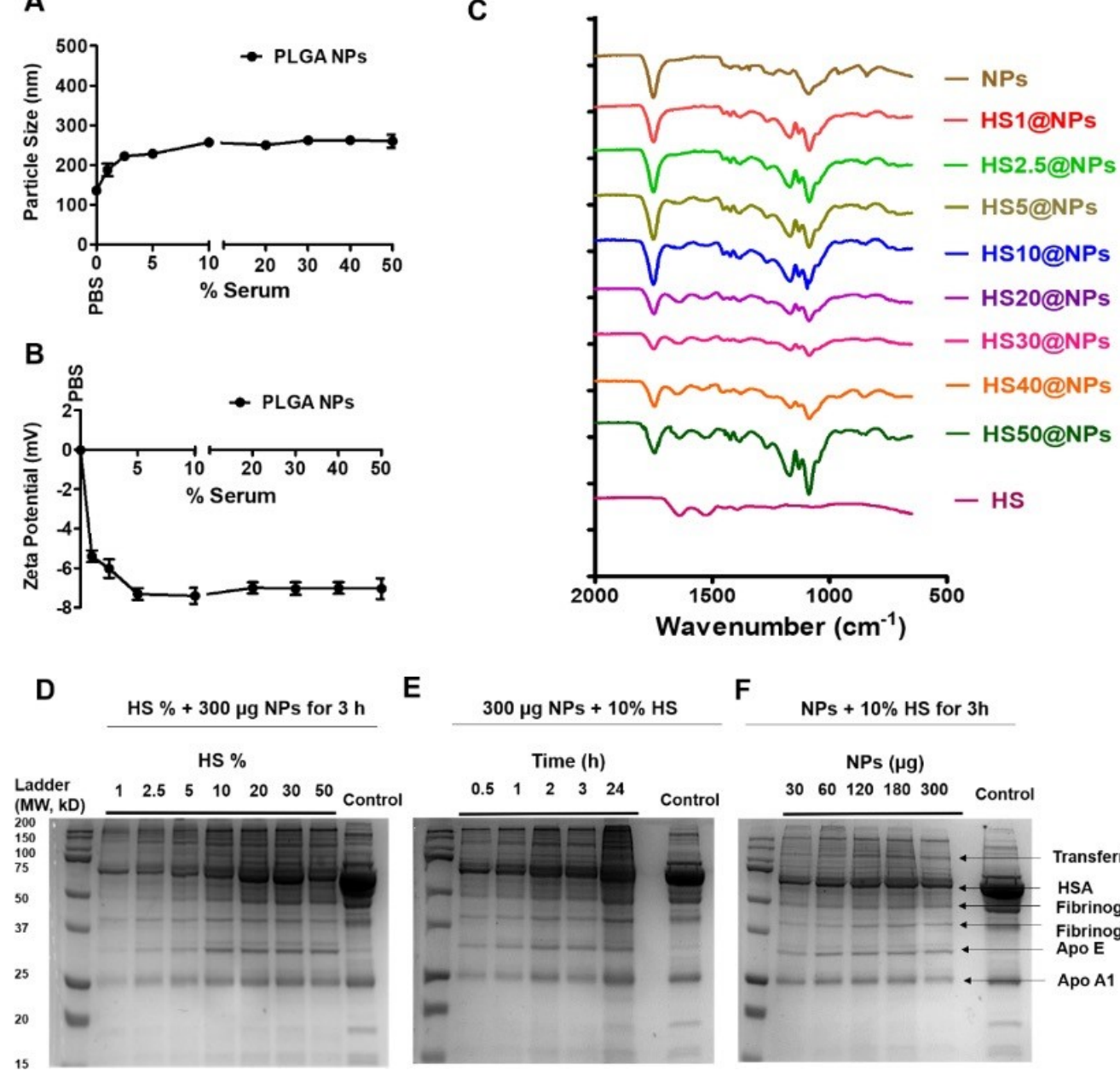

E

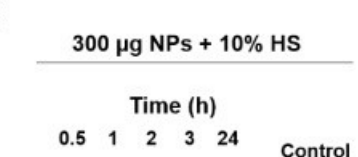

$\mathbf{F}$

NPs $+10 \%$ HS for $3 h$

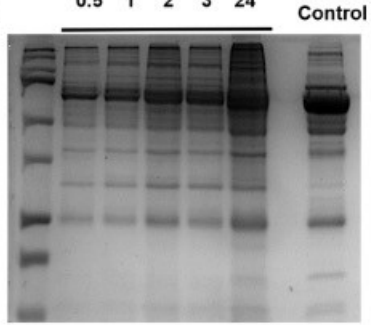

NPs $(\mu g)$ $\begin{array}{llllll}30 & 60 & 120 & 180 & 300 & \text { Control }\end{array}$

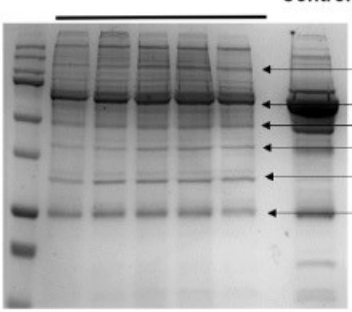

Transferrin

HSA

Apo $\mathrm{E}$

Apo A1

Fibrinogen beta

Fibrinogen gamma

Figure 4-5. Evaluation of human serum protein binding on PLGA NPs.

(A) Particle size of PLGA NPs in 1-50\% HS. (B) Zeta potential of PLGA NPs in 1-50\% HS. (C) FTIR spectra of HS, PLGA NPs and HS@NPs. (D-E) SDS-PAGE of adsorbed HS proteins on PLGA NPs. Data represents HS proteins binding to PLGA NPs dependent on the (D) concentration of HS, (E) incubation time, and (F) concentration of PLGA NPs. Mean \pm SEM values are from 3 independent experiments. 
NPs did not precipitate in the presence of HS. To confirm the HS binding on PLGA NPs, we performed a FTIR analysis of PLGA NPs, HS, and HS-bound NPs. The FTIR data was presented from 600 to $1800 \mathrm{~cm}^{-1}$ to observe the HS protein peaks. Peaks at $1661 \mathrm{~cm}^{-1}$ and $1541 \mathrm{~cm}^{-1}$ were found in HS and HS@NPs due to the amide bond stretch of the HS proteins. The peak extent of the HS proteins in HS@NPs showed a concentrationdependent increase with increasing percentage of HS (Figure 4-5C). Moreover, we further confirmed the PLGA NPs-protein interaction using SDS-PAGE analysis, in which, it can be observed the HS proteins binding on HS@NPs. The identified major proteins that bind on NPs are: transferrin, human serum albumin (HSA), fibrinogen beta, fibrinogen gamma, apolipoprotein E (Apo E), and apolipoprotein A1 (Apo A1), based on molecular weight [128]. The adsorbed HS proteins Apo E and Apo E have been shown to improve brain delivery of PLGA NPs [129]. We found that HS proteins were adsorbed to the surface of PLGA NPs when the concentration of HS increased (Figure 4-5D). With an increased incubation time, an increased protein binding on the PLGA NPs was also observed (Figure 4-5E). HS protein binding also showed an increased pattern when the amount of PLGA NPs was increased up to $180 \mu \mathrm{g}$ while after which HS protein binding decreased (Figure 4-5F). This indicates the saturation of binding with NPs due to the limited HS amount and incubation time.

\section{Hemocompatibility and Biocompatibility of PLGA-EVG NPs}

Hemocompatibility tests evaluate the effects on RBCs when a foreign compound is introduced to the blood. Both visual inspection (Figure 4-6A) and spectrophotometric measurement of hemoglobin release (Figure 4-6B) suggested that PLGA NPs do not cause hemolysis even at the highest concentration $(200 \mu \mathrm{M})$ that was used in the assay. A dose dependent hemolysis was observed in RBCs treated with EVG native drug. At the highest tested concentration $(200 \mu \mathrm{M})$, EVG native drug caused $\sim 30 \%$ hemolysis while PLGA-EVG NPs did not cause hemolysis. Similarly, HS30@EVG NPs also caused low hemolysis ( 4\%) compared to EVG native drug ( 30\%). Moreover, EVG native drug caused morphology change in RBCs, while PLGA-EVG NPs and HS30@EVG NPs caused no change in RBC morphology (Figure 4-6C). The hemocompatibility of PLGA NPs could be because of the biocompatibility of the polymer and the HS adsorption on the PLGA NPs.

It is important to carefully control the biocompatibility and cytotoxicity of a biomaterial for the potential use in the clinic [130]. Thus, we evaluated the toxicity profile of PLGA-EVG NPs with MDM and MMG. Over the range of 0-20 $\mu \mathrm{M} \mathrm{EVG,}$ PLGA-EVG or $30 \mathrm{HS} @$ EVG NPs, 100\% cell viability was observed with all these groups when compared to control cells (Figure 4-7A-B). No change in cell morphology was observed even after treating with the highest concentration $(20 \mu \mathrm{M})$ of EVG native drug, PLGA-EVG NPs, or HS30@PLGA-EVG (Figure 4-7C).

In conclusion, it is expected that HS protein binding occurs upon incubation with different percentage of HS due to the hydrophilic polymer PEG and hydrophilic groups

of poloxamer 188. The dynamic corona formed on PLGA NPs did not significantly alter 
A

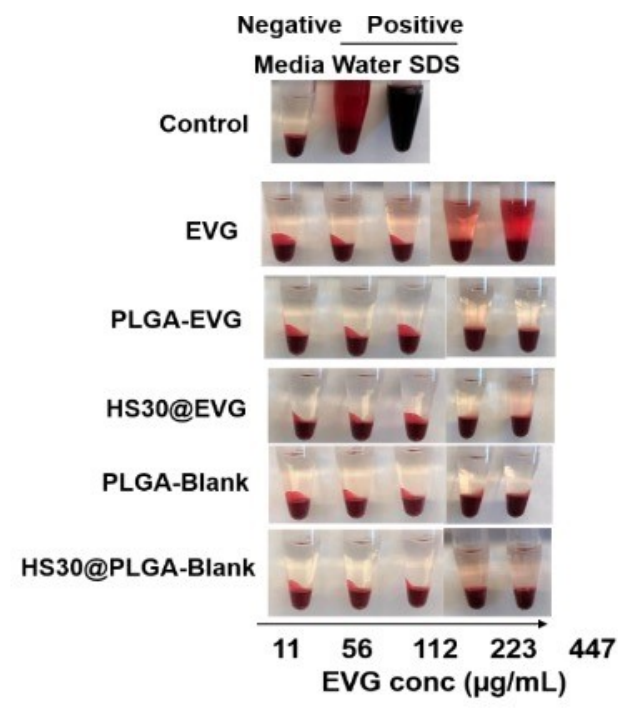

C

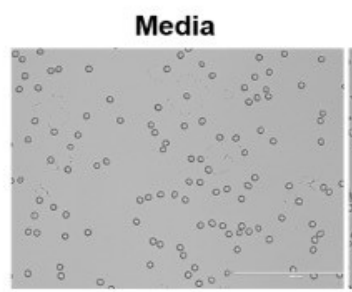

PLGA-EVG

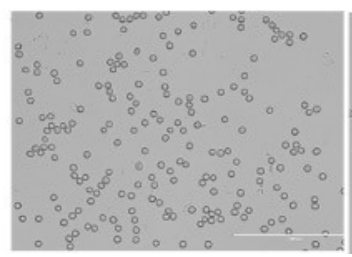

B

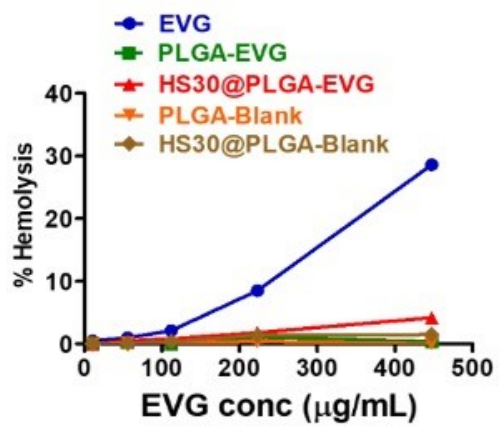

SDS

EVG

Figure 4-6. Hemocompatibility of PLGA NPs.

(A) Visual inspection of the tubes containing RBCs after exposure to 5-200 $\mu \mathrm{M} \mathrm{EVG}$, PLGA-EVG NPs, HS30@EVG NPs, Blank NPs, and HS30@Blank NPs. (B) Hemolysis of RBCs upon incubation with 5-200 $\mu \mathrm{M}$ EVG, PLGA-EVG NPs, HS30@EVG NPs, Blank NPs, and HS30@Blank NPs. (C) Microscopic images of RBCs treated with 447 $\mu \mathrm{g} / \mathrm{mL}$ EVG, PLGA-EVG NPs, HS30@EVG NPs, Blank NPs, and HS30@Blank NPs. 1X PBS was used as negative control, water was used as positive control \#1, and sodium dodecyl sulfate (SDS) was used as positive control \#2. \% of hemolysis was calculated based on the positive control \#1 (water). Data represents triplicates. 

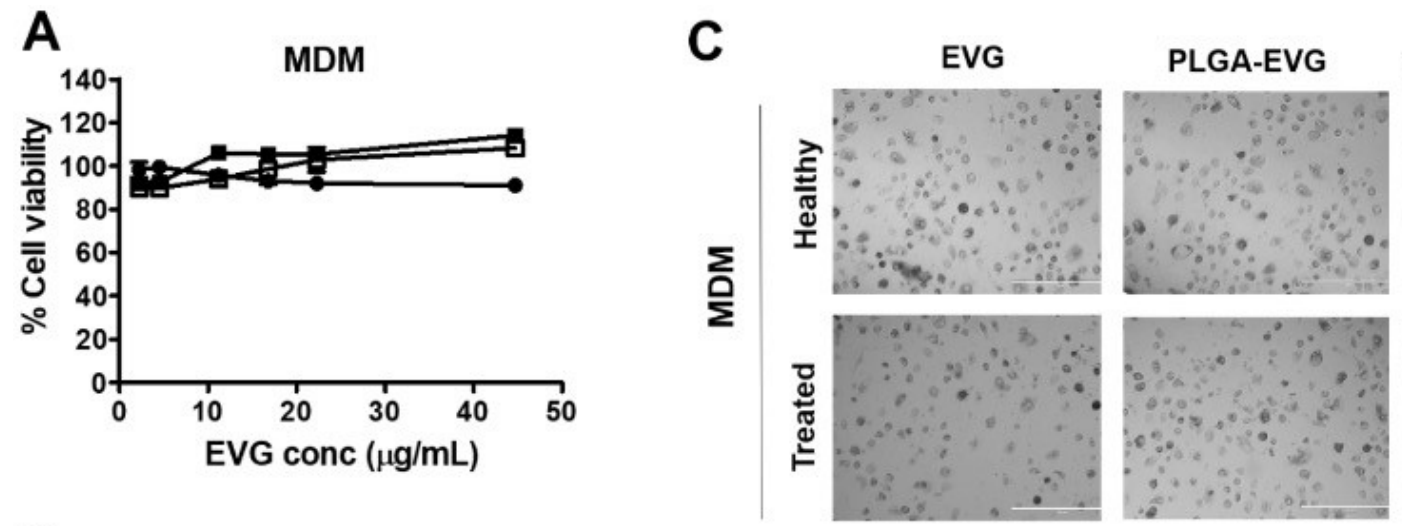

HS30@PLGA-EVG Positive control (toxicity)
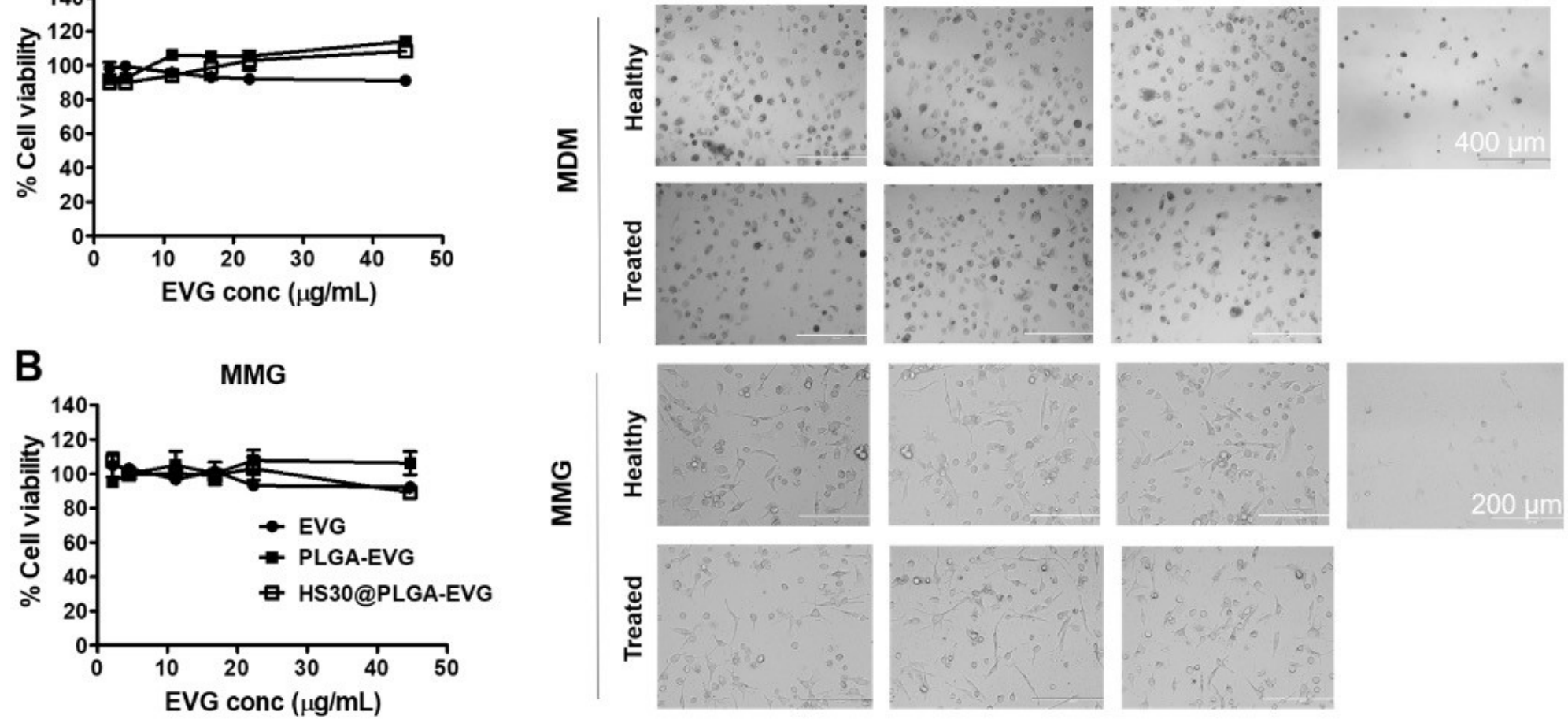

Figure 4-7. Toxicity evaluation of PLGA-EVG NPs in MDM and MMG.

(A-B) Cell viability of MDM and MMG with the treatment of 0-44.5 $\mu \mathrm{g} / \mathrm{mL}$ EVG, PLGA-EVG NPs, and 30\% HS bound-PLGA-EVG NPs complexes (HS30@PLGA-EVG), measured by XTT assay. (C) Microscopic images of MDM and MMG with the treatment of 0 and $44.5 \mu \mathrm{g} / \mathrm{mL}$ EVG, PLGA-EVG NPs, and HS30@PLGA-EVG. 5\% acetonitrile was used as a positive control. Mean \pm SEM values are from 5 replicates. 
the particle size of PLGA NPs, nor their hemocompatibility, or biocompatibility to macrophages and microglia, while it did alter zeta potential

\section{Internalization Mechanism of PLGA NPs in Macrophages and Microglia}

In the previous section, we showed a time- and concentration-dependent uptakes of PLGA NPs in monocytes. To further study the endocytosis pathways of these PLGA NPs in macrophage and microglia, various endocytosis inhibitors were utilized. The inhibitors include nocodazole, Cyto-D, CPZ, monensin, genistein, and $\mathrm{M} \beta \mathrm{CD}$, which suppress microtubule-related internalization, macropinocytosis, clathrin-mediated endocytosis, lysosome-involved internalization, caveolae-mediated pathways, and caveolae-/clathrin-mediated endocytosis, respectively $[131,132]$. To detect by flow cytometry, a fluorescence probe, C6 (green) was encapsulated in PLGA NPs. The inhibition effect of all the endocytosis inhibitors on the internalization of PLGA-C6 NPs in MDM are minor, indicating that the internalization of PLGA NPs in MDM may relate to a combination of endocytosis pathways (Figure 4-8A-B). In MMG cells, the cellular uptake of PLGA-C6 NPs was diminished by the compounds with the inhibition strength in the order of $\mathrm{M} \beta \mathrm{CD}>$ genistein $>\mathrm{CPZ}$. Among these inhibitors, $\mathrm{M} \beta \mathrm{CD}$ inhibited up to $55 \%$ uptake of PLGA-C6 NPs, which strongly indicates that the main internalization of PLGA NPs in MMG occurred through caveolae-/clathrin-mediated endocytosis (Figure 4-8C). We further examined the sub-cellular fate of PLGA NPs using a confocal microscopic analysis (Figure 4-8D). PLGA NPs were efficiently internalized in both MDM and MMG after $3 \mathrm{~h}$ exposure. A co-localization of PLGA-C6 and early endosome markers were observed in both MDM and MMG cells, but less co-localization was observed in the presence of late-endosomal marker, and no co-localization was observed in the presence of the lysosomal or mitochondria marker. This suggests that PLGA NPs can escape from endo-lysosomal compartments and deliver the therapeutics to the cells efficiently.

Understanding the mechanism of internalization of NPs by the targeted cells can increase our knowledge of how these particles are taken up and are transported within the cells, and to what extent they are secreted [133]. In general, most of the NPs are taken up into cells via endocytosis into endosomes and subsequently trafficked into lysosomal compartments [134]. Literature reports have suggested that polymeric NPs are taken up by mouse primary microglia mainly by clathrin-mediated endocytosis [135-137]. Similarly, we observed that the caveolae-/clathrin-mediated endocytosis serves as the major pathways for human MMG to uptake PLGA NPs. In contrast to the microglia, however, our data showed that a combination of different endocytosis pathways are involved in the uptake of PLGA NPs into the macrophages, which is also consistent with previous studies [138-140]. Although initially present in early endosomes, PLGA NPs can still bypass the endo-lysosomal compartments in both MDM and MMG with less and no co-localization found in the presence of late endosomal and lysosomal markers, respectively. Our design allows the PLGA NPs to escape the endo-lysosomal degradation/secretion, which helps the therapeutic to work effectively inside the 


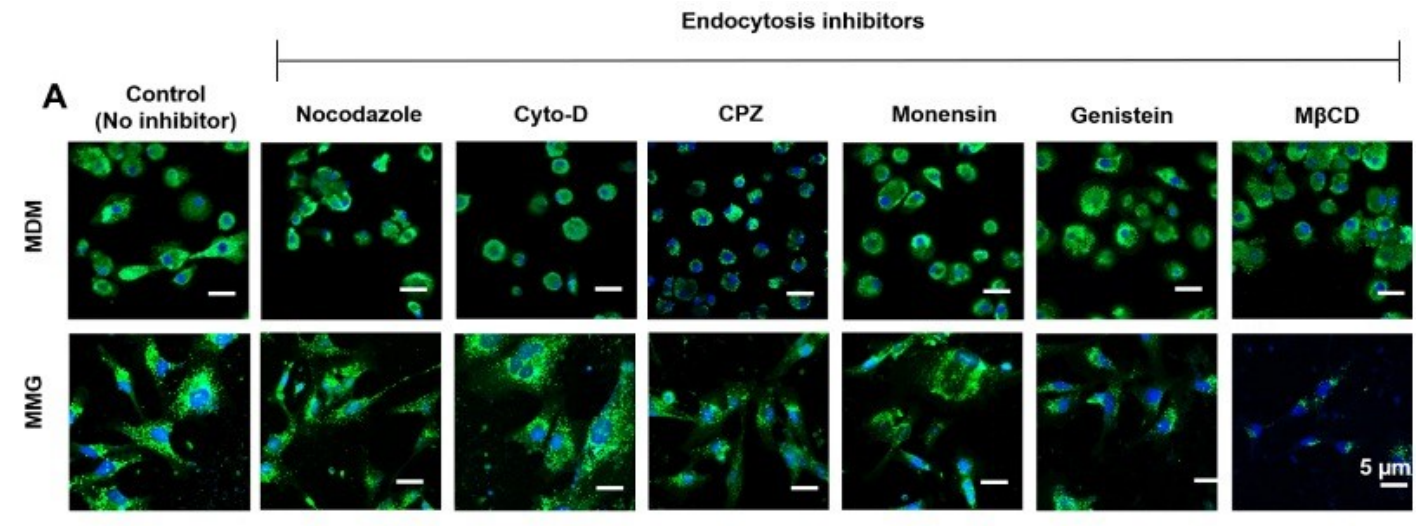

B
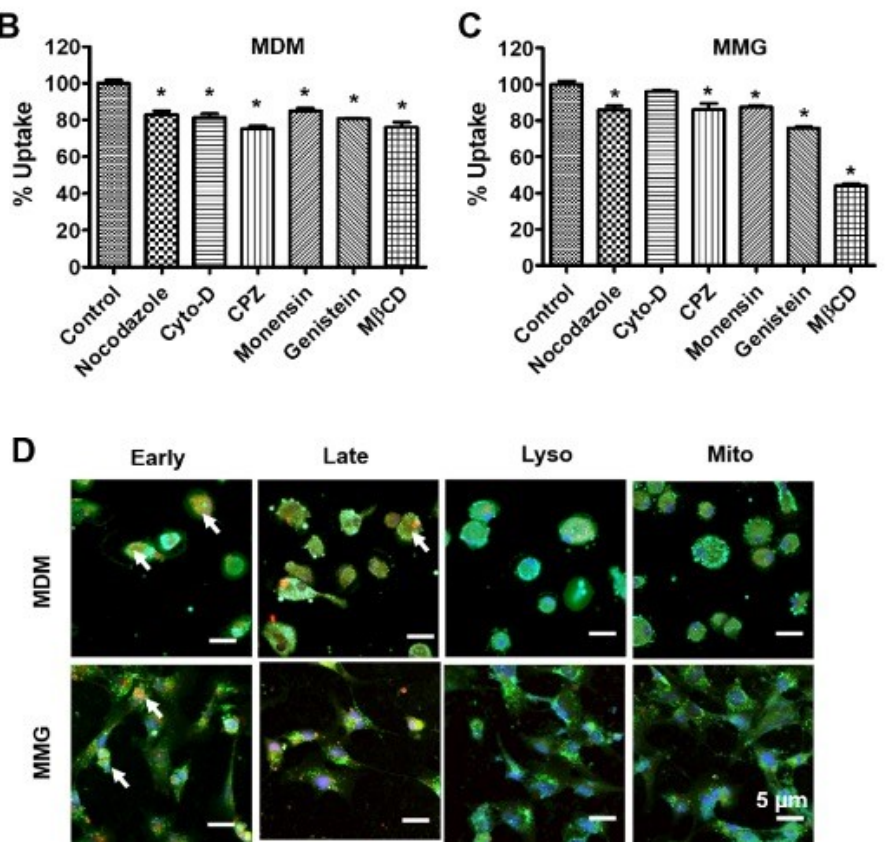

Figure 4-8. Internalization mechanism of the PLGA-C6 NPs in MDM and MMG. (A) Cellular uptake of PLGA-C6 NPs in the presence of various endocytosis inhibitors. (B-C) Relative cellular uptake percentage calculated from the mean fluorescence intensity measured by flow cytometry in MDM and MMG. Mean \pm SEM are from 3 measurements. ${ }^{*} p<0.05$. (D) Co-localization evaluation of PLGA-C6 NPs (green) in presence of markers (red) for early endosome, late endosome, lysosome, and mitochondria. DAPI was used to stain nuclei (blue). White arrow indicates the colocalization of PLGA-C6 NPs with early- and late- endosome markers in the cells. Cells were visualized under $400 \times$ magnification $(\operatorname{Bar}=5 \mu \mathrm{m})$. 
cells [134]. To the best of our knowledge, this is the first report studying the mechanistic internalization of NPs by human microglia.

\section{EVG Nanoformulation for Drug Delivery Across the BBB to Achieve HIV-1 Suppression in the CNS}

As described before, the introduction of antiretroviral therapy (ART) has made significant advances in managing HIV-1/AIDS effectively [1]. However, the prevalence of HIV-1-associated neurocognitive disorders (HAND) is still a major concern, especially in the aging population [2]. The key cells that support productive HIV-1 infection and replication in the brain are macrophages and microglia $[8,9]$. Therefore, maintaining a therapeutic concentration of ARVs in HIV-1-infected macrophages and microglia is important for an effective HIV-1 treatment in the brain. Further, the expression of the efflux transporters P-gp, and the drug metabolic enzyme, CYP3A4 in macrophages and microglia makes it more difficult to maintain the therapeutic levels of ARVs in these cells using conventional regimens [77-79].

NP-based drug delivery systems provide a relatively safe profile, protects ARVs from efflux transporters as well as from enzymatic and hydrolytic degradations, and can be used for a sustained-release of therapeutics [40]. NPs that have been studied for brain delivery mainly include polymeric NPs such as poly(glycolic acid) (PGA), PLA, PLGA, and PBCA NPs [41, 42], and metal-based NPs such as gold, silver, and zinc oxide NPs [42]. Since polymeric NPs are not only easy to be prepared and stored but also highly biocompatible and biodegradable in nature, polymeric NPs have been extensively studied for delivery of therapeutics for cancer [43-46] and HIV-1 [47-50]. However, the use of polymeric NPs to deliver ARVs to the brain for the treatment of HAND is still underdeveloped.

To utilize PLGA NPs as a potential tool for drug delivery to the brain, in this chapter, we performed the transmigration study of PLGA NPs in an in vitro BBB model

and in vivo mouse models, and subsequently assessed the efficacy of PLGA-EVG NPs on viral suppression of HIV-1-infected primary macrophages and microglia after crossing the BBB. Overall, PLGA NPs provided a promising method for delivery of EVG across the $\mathrm{BBB}$ and have a potential for therapeutic interventions in reducing HAND.

\section{In Vitro BBB Model and Transendothelial Electrical Resistance Assessments}

The in vitro $\mathrm{BBB}$ model used in this study was co-cultured endothelial cells (bEnd.3) with astrocytes (C8-D1A) in a Transwell ${ }^{\circledR}$ plate as described in Chapter 3 (Figure 4-9A). The bEnd.3 cell are a commercially available cell line and a useful BBBmimicking system for biological and pharmacological research because they expresses the efflux transporters P-gp and breast cancer resistance protein (BCRP) [141, 142]. A bEnd.3/C8-D1A co-culture model has shown to present significantly higher development 
A

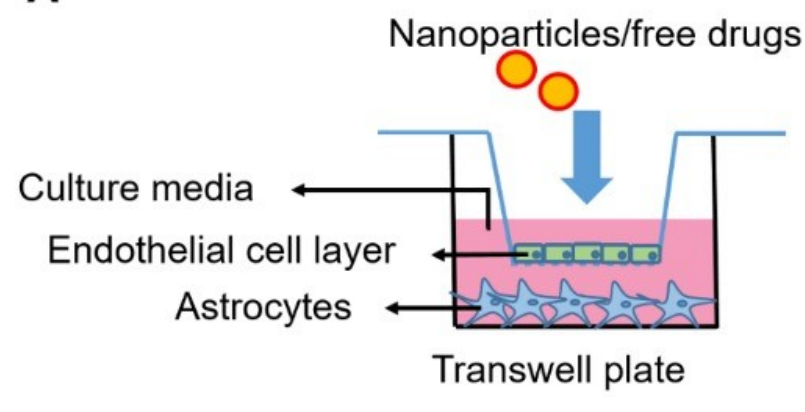

B

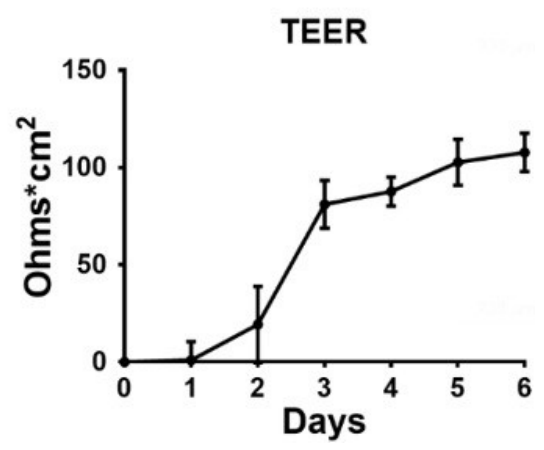

Figure 4-9. In vitro BBB model.

(A) Graphical representation of the in vitro BBB model. (B) TEER measurements were obtained by applying a transendothelial current to the membrane and then testing the resistance (current, Ohm) multiplied by the area $\left(\mathrm{cm}^{2}\right)$ of the endothelial monolayer $\left(\mathrm{Ohm} \cdot \mathrm{cm}^{2}\right)$ by using an EVOM2 meter. Mean \pm SEM values were graphed from 3 independent experiments. 
of tight junctions than the monoculture of bEnd.3 [89]. In our study, transendothelial electrical resistance (TEER) values were measured for 6 days after co-culturing. We observed that the TEER value reached a plateau on day-4 and it was stable from 4 to 6 days (Figure 4-9B). A mean TEER value of 100 to $120 \mathrm{Ohm} \cdot \mathrm{cm}^{2}$ was observed in the confluent BBB model, which is consistent with literature reports [89]. All transmigration experiments were conducted after membrane integrity was stable with consistent TEER values.

\section{Transmigration of PLGA NPs Across the In Vitro BBB}

The characterization of the PLGA-C6 NPs was reported previously, which demonstrated a time- and dose-dependent uptakes in monocytes [143]. To test the transmigration of PLGA-C6 NPs across the in vitro BBB, three different concentrations of PLGA-C6 NPs were exposed in the upper chamber, and the culture media in the bottom chamber was collected after 24 hours. The penetration of PLGA-C6 NPs was examined by quantifying the fluorescence intensity of the culture media, which was collected from the bottom chamber. We observed a dose-dependent increase in fluorescence intensity from media samples, which suggests a dose-dependent penetration of PLGA-C6 NPs across the BBB (Figure 4-10A). This finding is also supported by a quantification of $\mathrm{C} 6$ from the same media. $\mathrm{C} 6$ concentrations in the media collected from the bottom chamber were calculated using a $\mathrm{C} 6$ calibration curve. The penetration of $\mathrm{C} 6$ across the BBB also followed a dose-dependent profile, and all the PLGA-C6 groups showed significant increase in C6 fluorescence intensity compared with PLGA-Blank after crossing the BBB (Figure 4-10B, ${ }^{*} \mathrm{p}<0.05$ compared with PLGA-Blank, $\# \mathrm{p}<0.05$ compared with the previous concentration). These results were also validated by the qualitative imaging of recipient astrocytes, which showed that PLGA-C6 NPs were taken up by cells in a dose-dependent manner after crossing the BBB. The highest fluorescence was observed in cells treated with the highest concentration of PLGA-C6 NPs (Figure 410C).

\section{Increased Penetration of PLGA-EVG NPs in the In Vitro BBB Model and In Vivo Mouse Model}

To understand whether nanoparticle formulation can increase the transmigration of EVG in the in vitro BBB model, we measured EVG concentrations in the bottom chamber from both media and cell lysate samples. As expected, PLGA-EVG NPs showed higher penetration compared to EVG-free drug. Dose-dependent penetration profiles for both EVG-free drug and PLGA-EVG NPs were observed. A significant increase in EVG penetration was observed for both native EVG and PLGA-EVG NPs from $2.5 \mu \mathrm{g} / \mathrm{mL}$ to 5 $\mu \mathrm{g} / \mathrm{mL}$ and from $5 \mu \mathrm{g} / \mathrm{mL}$ to $10 \mu \mathrm{g} / \mathrm{mL}$ (Figure 4-11A, ${ }^{*} \mathrm{p}<0.05$ compared with PLGABlank, $\# \mathrm{p}<0.05$ compared with the previous concentration). The results showed that a maximum of $27 \%$ of PLGA-EVG NPs can cross the BBB after 24 hours. The results also showed a significant increase in penetration of PLGA-EVG NPs compared to EVG native drug at the dose of $5 \mu \mathrm{g} / \mathrm{mL}$ and $10 \mu \mathrm{g} / \mathrm{mL}$. We also performed a time-dependent 

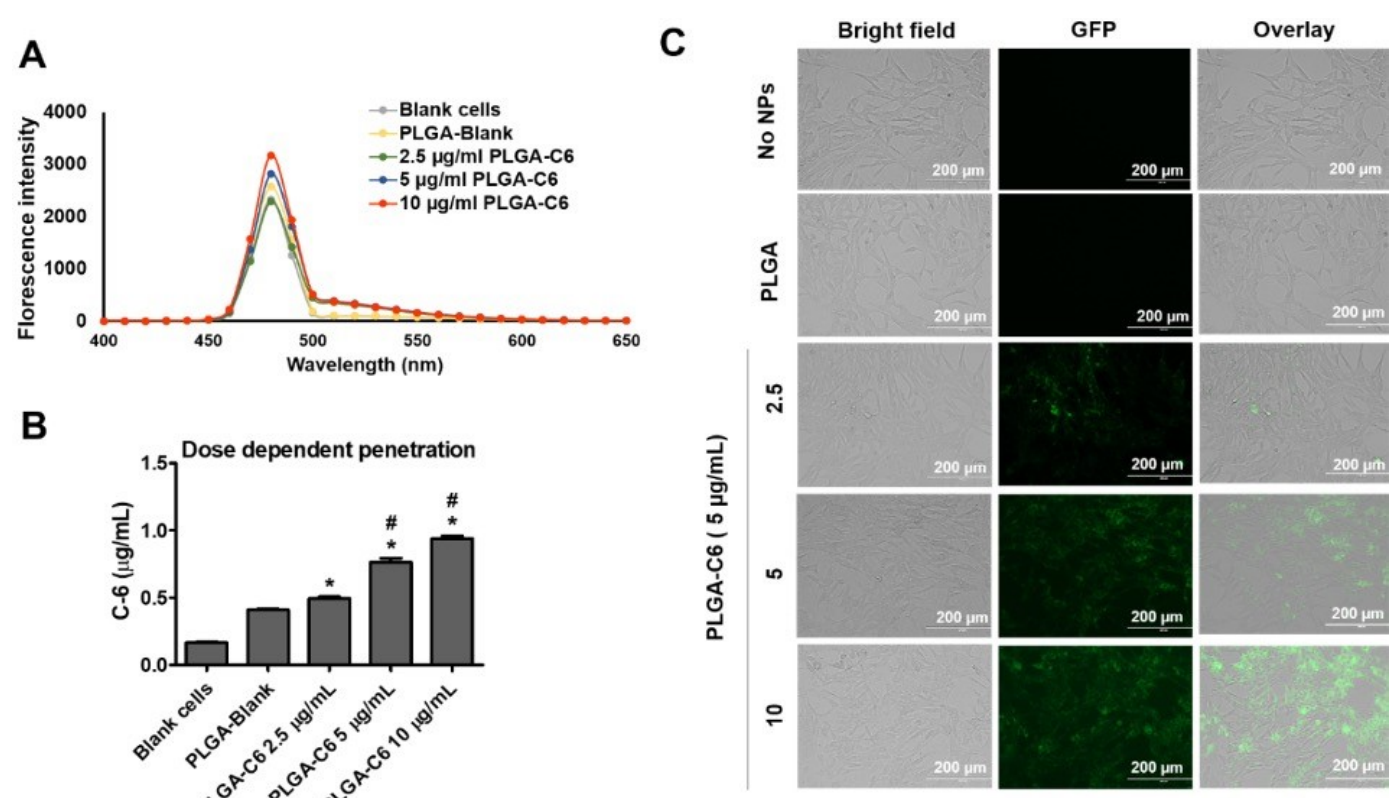

Figure 4-10. Dose dependent penetration of PLGA NPs across the BBB in vitro. (A) Quantitative penetration of PLGA-C6 after 24 hours exposure of PLGA-C6 to the top insert. The florescence intensities of the media from bottom chambers was determined by SpectraMax M2e UV spectrometer. Fluorescence was studied by excitation at $488 \mathrm{~nm}$ and emission between 400-650 nm. (B) C6 concentration in the media was calculated using a C6 calibration curve with the matrix of media. C6 concentration of $1 \mu \mathrm{g} / \mathrm{mL}$ represents 302101 mean florescence intensity (area under the curve). (C) Bright field, fluorescent, and overlay images of astrocytes from bottom chambers after 24 hours exposure of PLGA-C6 to the top insert. PLGA-C6 was taken up dose-dependently by the astrocytes in bottom chambers after crossing the BBB. The green florescence was visualized using an EVOS ${ }^{\circledR}$ FL Imaging System. Scale bar $=200 \mu \mathrm{m}$. Mean \pm SEM values were graphed from 3 measurements. * indicates $\mathrm{p}<0.05$ compared with PLGABlank, \# indicates $\mathrm{p}<0.05$ compared with the previous concentration. 
Figure 4-11. Dose-/time-dependent penetration of EVG and PLGA-EVG NPs across the $\mathrm{BBB}$ in vitro.

(A) Dose dependent penetration profile of EVG and PLGA-EVG NPs across the BBB in vitro. (B) Time dependent penetration profile of EVG and PLGA-EVG NPs across the in vitro BBB. Quantitative penetration of EVG was determined by LC-MS/MS. (C)TEER of the endothelial cell layer was measured before and after treatment. * indicate $p<0.05$ compared with native EVG drug, \# indicate $\mathrm{p}<0.05$ compared with the previous EVG/PLGA-EVG concentrations. (D) Microscopic images of the endothelial cells from the top insert were taken before and after treatment using an EVOS $®$ FL Imaging System. Scale bar $=200 \mu \mathrm{m}$. (E) Time course penetration profile of EVG or PLGA EVG NPs across the in vitro BBB in the presence or absence of the P-gp inhibitor elacridar. (F) Area under the curve of EVG and PLGA-EVG penetration in presence and absence of Pgp inhibitor elacridar. Area under the curve from 0 to last time $t\left(\mathrm{AUC}_{0-\mathrm{t}}\right)$ was calculated using the linear trapezoidal method. Mean \pm SEM values were graphed from 3 independent experiments. * indicates $\mathrm{p}<0.05$ compared with native EVG drug in absence of the P-gp inhibitor. 
A

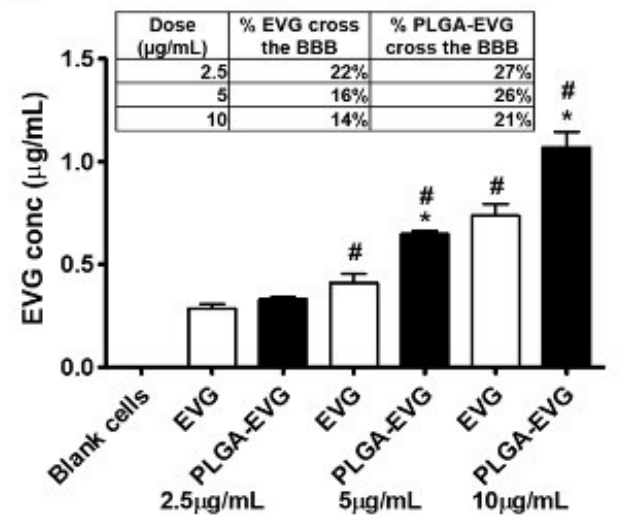

C

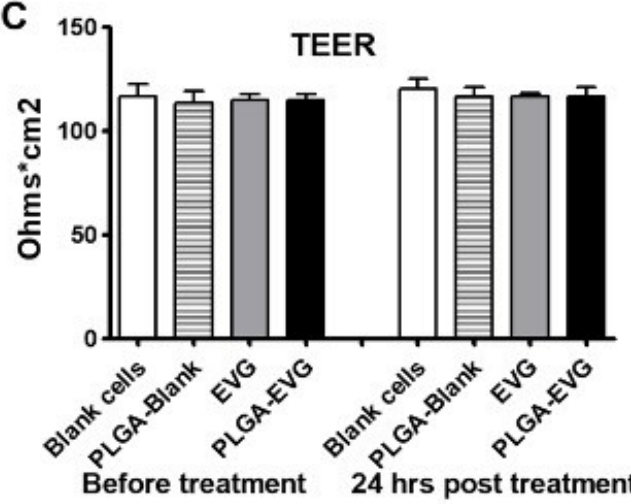

E

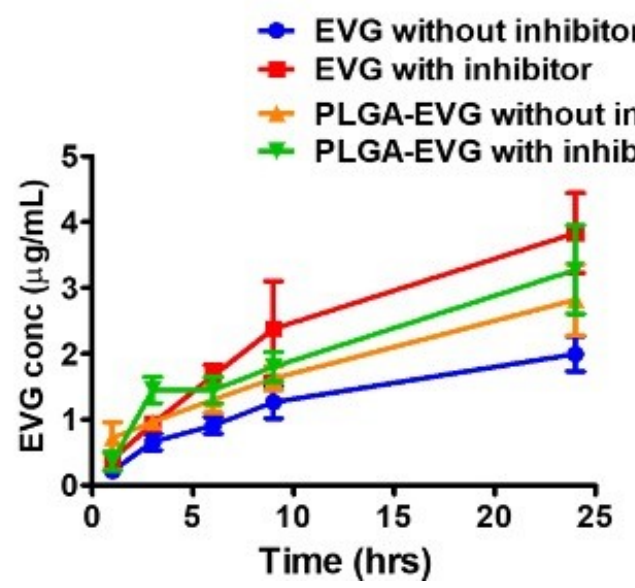

B Time dependent penetration

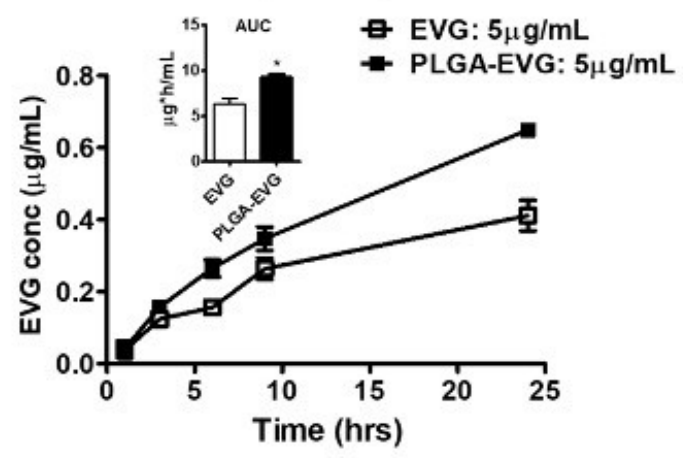

D Microscopic images of the top insert

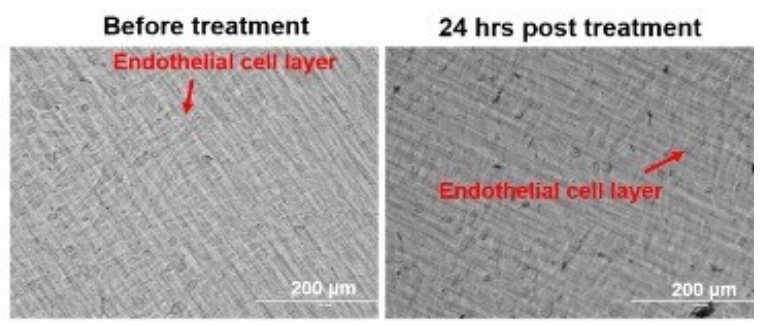

$\mathbf{F}$

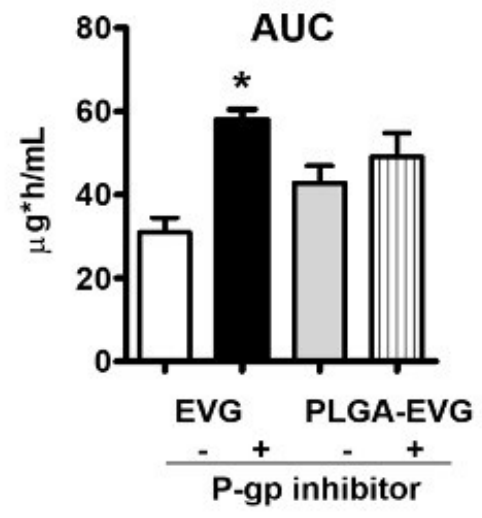


penetration study using $5 \mu \mathrm{g} / \mathrm{mL}$ of native EVG/PLGA-EVG NPs. Both native drug and NPs showed a time-dependent penetration across the BBB model. Penetration of PLGAEVG NPs across the BBB showed a significant increase in the area under the curve compared to EVG native drug (Figure 4-11B, ${ }^{*} p<0.05$ ). These results demonstrate that PLGA-EVG NPs improve the ability of EVG to cross the BBB in the in vitro model. Moreover, no changes were observed in TEER values (Figure 4-11C) or endothelial cell morphology (Figure 4-11D) before and after the treatment, suggesting that PLGA-EVG transmigration did not change the BBB monolayer integrity. Additionally, to identify the mechanistic contribution of $\mathrm{P}$-gp in interfering the penetration of EVG in the in vitro BBB model and the capability of PLGA NPs to bypass the BBB efflux transportation. We performed a penetration study in presence and absence of the P-gp inhibitor elacridar for 24 hours (Figure 4-11E). The $\mathrm{AUC}_{(0-\mathrm{t})}$ of in vitro $\mathrm{BBB}$ penetration for $\mathrm{EVG}$ increased significantly after in the presence of the P-gp inhibitor, which indicates that the low EVG level in the CNS is partially mediated by P-gp-mediated efflux (Figure 4-11F). In contrast, the penetration of PLGA-EVG NPs showed no difference between the presence or absence of the P-gp inhibitor, demonstrating the ability of NP to bypass the efflux transporter P-gp in the in vitro BBB.

The bioavailability and brain permeability of EVG loaded on PLGA NPs were evaluated by comparing the plasma and brain concentrations of native EVG with PLGAEVG NPs. We prepared calibration curves using blank plasma and blank brain homogenates to quantify plasma samples and brain samples to minimize the matrix effect, respectively. The linear calibration curve was achieved over the range of $1-500$ $\mathrm{ng} / \mathrm{mL}$ with a weighting factor of $1 / \mathrm{y}$ and a correlation coefficient $\left(\mathrm{r}^{2}\right)$ of 0.999 for plasma EVG (Figure 4-12A). The calibration curve for brain homogenates followed a linear regression over the range of $1-125 \mathrm{ng} / \mathrm{mL}$ with a weight factor of $1 / \mathrm{y}$ and $\mathrm{r}^{2}$ of 0.997 (Figure 4-12B). The mean plasma concentration versus time profiles of PLGA-EVG NPs and native EVG after intraperitoneal (i.p.) injection is shown (Figure 4-12C, ${ }^{*} \mathrm{p}<0.5$ ). The calculated mean plasma $\mathrm{C}_{\max }$ of PLGA-EVG NPs was $186 \mathrm{ng} / \mathrm{mL}$, which is approximately 6 -fold greater than the $\mathrm{C}_{\max }$ achieved by the native drug $(27.2 \mathrm{ng} / \mathrm{mL})$. The AUC $_{(0-\mathrm{t})}$ of the PLGA-EVG NPs was $1911 \mathrm{ng} * \mathrm{~h} / \mathrm{mL}$, which is significantly higher than the native drug. The higher $\mathrm{C}_{\max }$ and $\mathrm{AUC}_{(0-\mathrm{t})}$ indicate that the nanoformulation increases the bioavailability of EVG compared to the same dose of the free drug in mouse plasma. Mouse brain concentrations were also measured after sacrificing the mouse at $96 \mathrm{~h}$ to analyze the permeability of EVG across the BBB. Brain EVG concentration was found to be $\sim 3$-fold higher in PLGA-EVG NPs compared with the native drug (Figure 4-12D, $* p<0.5)$. Overall, the data showed an improved delivery of EVG to the brain using the PLGA NPs. Mice were healthy after injecting EVG native drug/PLGA-EVG NPs. No adverse events, weight loss, or animal dead was observed during the study.

NPs have been demonstrated to be a promising strategy to deliver drugs at the site of action, bypass transporters and drug metabolic enzymes, and cross the BBB [47, 49]. Thus, we hypothesize that, EVG in PLGA NPs can bypass transporters on the BBB to produce higher drug transmigration and can also bypass those same transporters and drug metabolic enzymes in macrophages and microglia after crossing the BBB, showing 
A
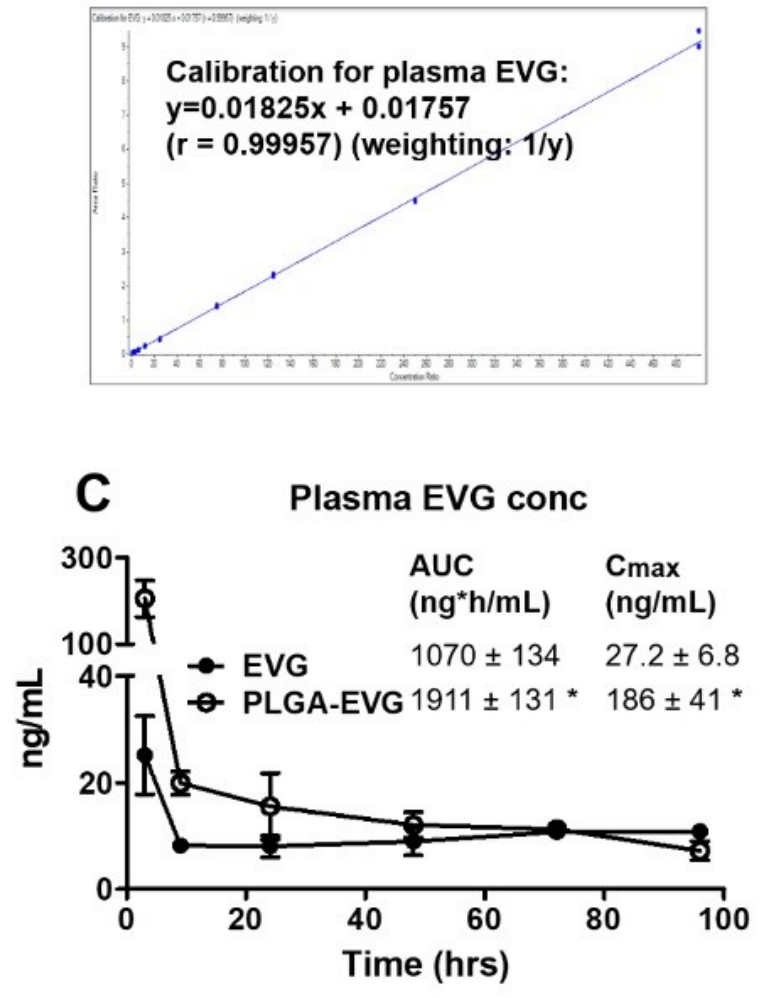

B

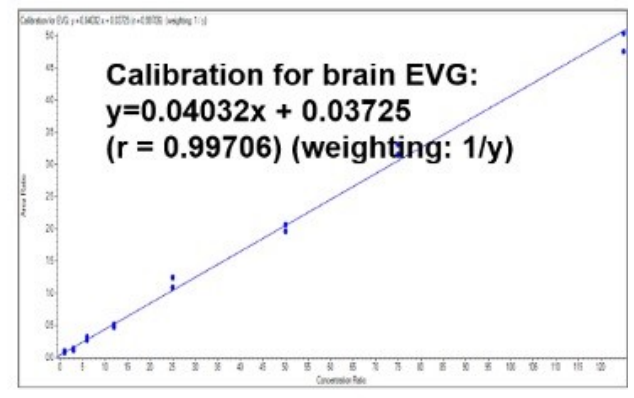

D

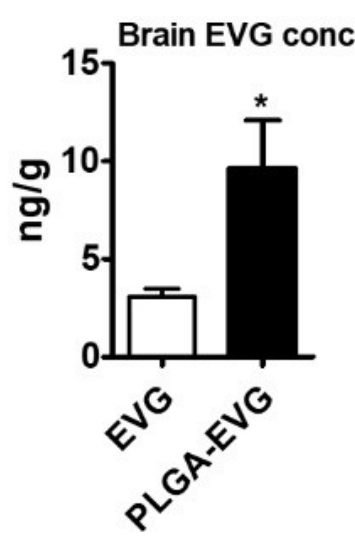

Figure 4-12. Plasma and brain concentrations of EVG and PLGA-EVG NPs in mice. (A) LC-MS/MS calibration curve for EVG in plasma samples from MultiQuant Software. (B) LC-MS/MS calibration curve for EVG in brain samples from MultiQuant Software. (C) Plasma concentrations of EVG were measured after i.p. administration of $20 \mathrm{mg} / \mathrm{kg}$ dose of native EVG $(n=8)$ or PLGA-EVG $(n=8)$ to mice by LC-MS/MS. Aera under the curve (AUC) and maximum concentration $\left(\mathrm{C}_{\max }\right)$ was analyzed using noncompartmental analysis by PK solver. (D) Brain concentrations of EVG were measured after sacrificing mice at $96-\mathrm{hr}$ time point. Mean \pm SEM values were graphed from eight mice measurements for each group. *indicates $p<0.05$ compared to EVG native drug. 
improved viral suppression in those cells. In order to have an efficient brain delivery, we used poloxamer 188 as the surfactant in our PLGA NP formulation. Poloxamer 188 has been used as an effective surfactant for brain delivery in cancer treatment $[80,145]$. Literature reports have shown that poloxamer 188 coating can help PLGA NPs become an efficient brain delivery method [80]. They demonstrated that poloxamer 188-coated PLGA NPs have a high anti-tumor effect against an intracranial 101/8 glioblastoma in rats, compared with the non-coated NPs. In another study, poloxamer 188-coated PLGA NPs demonstrated the greatest cellular uptake in brain tissues when compared with NPs coated with other commonly used surfactants such as polysorbate 80 and poloxamer 407 [145]. Similarly, in our study, the poloxamer 188-coated PLGA-EVG NPs demonstrated an effective brain delivery profile in our study. The poloxamer 188-coated PLGA-EVG NPs showed more than 50\% higher penetration of the in vitro BBB model compared with the native drug. Most importantly, this PLGA-EVG NPs also increase the EVG brain penetration by 3 -fold in the in vivo mouse model, compared with the native EVG.

\section{Improved Viral Suppression in HIV-1-Infected Macrophages and Microglia after Crossing the In Vitro BBB}

Since macrophages can be infected productively by HIV-1 in the CNS [8], we examined the viral suppression efficacy of PLGA-EVG NPs in HIV-1-infected macrophages. We examined PLGA-EVG NPs and native EVG for their effects on viral replication on HIV-1-infected MDM in a modified in vitro BBB model following a onemonth treatment paradigm as described in the treatment scheme (Figure 4-13A). HIV-1infected MDM were cultured on the bottom chamber, which was not exposed to EVG directly. Blank PLGA NPs, EVG, and PLGA-EVG NPs were added on the upper chamber, which contains the BBB monolayer. TEER values of the BBB model were measured for all the groups daily. Both EVG and PLGA-EVG NPs did not compromise the physiological integrity of the in vitro BBB model (Figure 4-13B). HIV-1 p24 protein levels were measured from the bottom chamber daily. Thus, any effects on viral suppression in the recipient MDM are the result of successful transmigration of the in vitro BBB by EVG native drug or PLGA-EVG NPs. Our results showed that PLGABlank NPs had no effect on viral replication. Both native EVG and PLGA-EVG NPs suppressed the viral replication to $70-100 \%$ of control, and an increased antiviral efficacy of PLGA-EVG NPs compared with EVG native drug on all the measured days (Figure 4-13C). We calculated the p24 area under the curve to compare the viral suppression efficacy of native EVG and PLGA-EVG NPs from day-1 to day-7 (Figure 413D, $\left.{ }^{*} \mathrm{p}<0.5\right)$. The results showed that the HIV-1-infected MDM, when exposed to PLGA-EVG NPs have a significantly lower viral load, indicating that PLGA-EVG NPs have better efficacy on viral suppression in HIV-1-infected MDM after crossing the in vitro $\mathrm{BBB}$ than $\mathrm{EVG}$ alone.

Other than macrophages, microglia also serve as a sanctuary site for HIV-1 in the CNS, which cannot be suppressed efficiently [10]. A one-month treatment paradigm for collecting HIV-1-infected MMG and performing EVG/PLGA-EVG viral suppression 


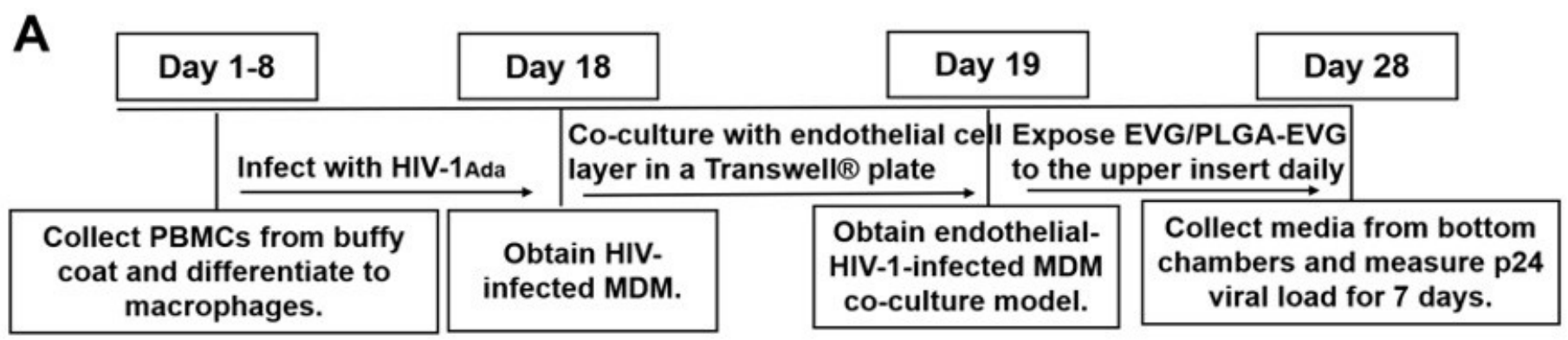
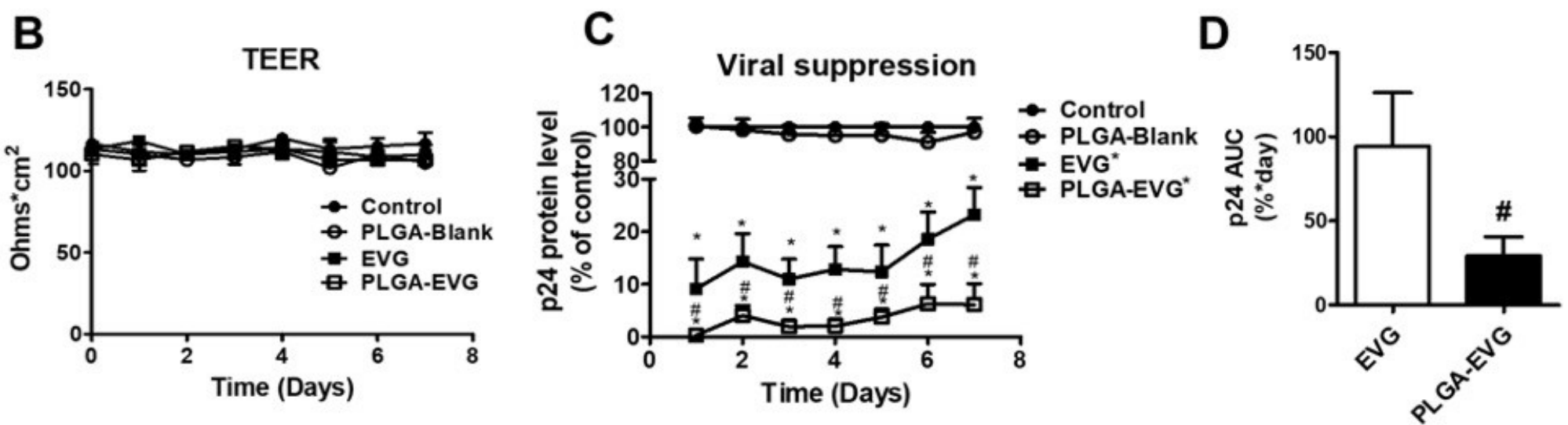

Figure 4-13. Viral suppression of EVG and PLGA-EVG in HIV-1-infected MDM after crossing an in vitro BBB model. (A) Treatment paradigm for collecting HIV-1-infected MDM and performing an EVG/PLGA-EVG viral suppression study. (B) TEER of the endothelial monolayer was measured at indicated time points. (C) Time course of HIV-1 replication upon drug exposure in HIV-1-infected MDM after crossing in vitro BBB model. All assays were performed on triplicate samples with MDM derived from two different donors. To minimize inter-donor variability, p24 levels were normalized to the control MDM, which were obtained from the same donor, and reported as a percentage of the control group. (D) Area under the curve of EVG and PLGA-EVG. Area under the curve from 0 to last time $\mathrm{t}\left(\mathrm{AUC}_{0-\mathrm{t}}\right)$ was calculated using the linear trapezoidal method. Mean $\pm \mathrm{SEM}$ values were graphed from triplicate samples with MDM derived from two different donors. *indicates $\mathrm{p}<0.05$ compared to control, \#indicates $\mathrm{p}<0.05$ compared to EVG native drug. 
study was used as described in the scheme (Figure 4-14A). Although the in vitro MMG is not representative of human primary microglia, MMG have shown to express microglia markers and possess similar morphological structure as human primary microglia [84]. As in the MDM study, the drug was exposed to the endothelial monolayer on the top of HIV-1-infected MMG, and HIV-1 viral load was measured from the bottom chamber, which was not exposed to EVG directly. Also, as in the MDM study, we did not observe monolayer integrity changes during the 7-day treatment (Figure 4-14B). PLGA-Blank showed no effect on viral load, compared with control cells. Both EVG and PLGA-EVG NPs showed significant HIV-1 suppression in HIV-1-infected MMG after crossing the BBB, while EVG showed no significant HIV-1 suppression on day 2. Moreover, PLGAEVG NPs showed a better viral suppression efficacy on day 1, 4, 5, and 6, compared with EVG native drug (Figure 4-14C). The p24 AUC showed the relative ability of viral suppression for EVG free drug and PLGA-EVG NPs. PLGA-EVG NPs showed $\sim 25 \%$ more viral suppression in HIV-1-infected MMG after crossing the in vitro BBB (Figure 4-14D, $\left.{ }^{*} \mathrm{p}<0.5\right)$.

Currently, magnetic nanoformulation is the most commonly studied nanoformulation for NeuroAIDS [55]. Magnetic NPs of azidothymidine showed a 3-fold higher transmigration in an in vitro BBB model, compared to the native drug [55]. This magnetic nanoformulation of azidothymidine also showed a slightly lower p24 antigen production when treating with HIV-1-infected PBMCs. The same group also developed a magnetic layer-by-layer NPs with a $\sim 37.95 \%$ BBB transmigration and $\sim 33 \%$ p 24 suppression effects on astrocytes in vitro [56]. Compared with those magnetic NPs, our PLGA-EVG NPs showed a comparable percentage of transmigration and viral suppression in vitro. 


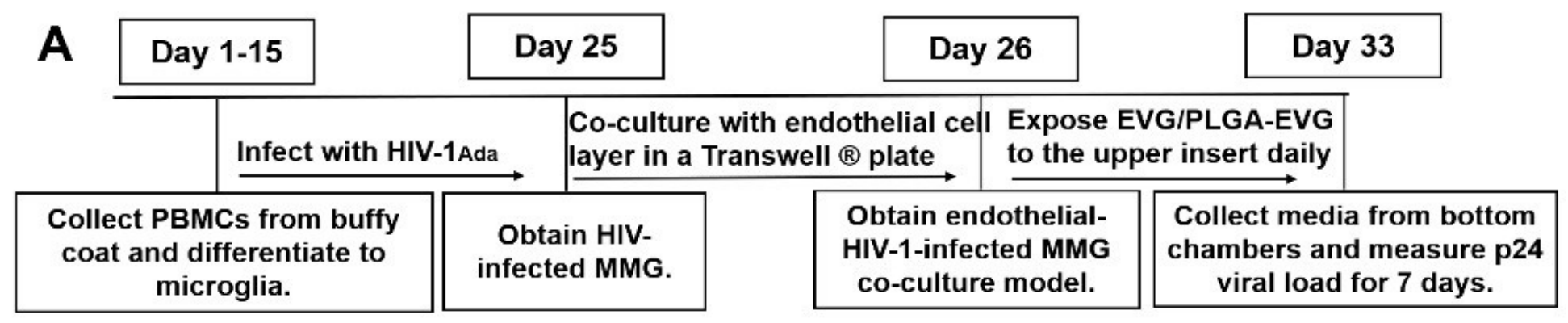

B

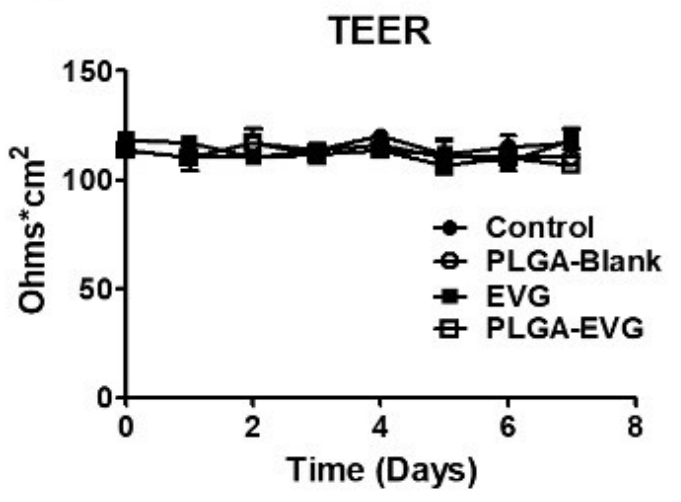

C

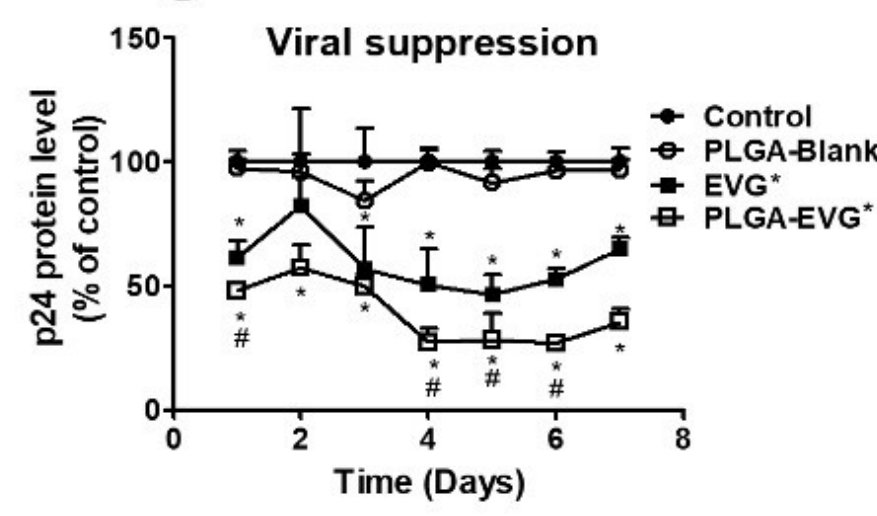

D

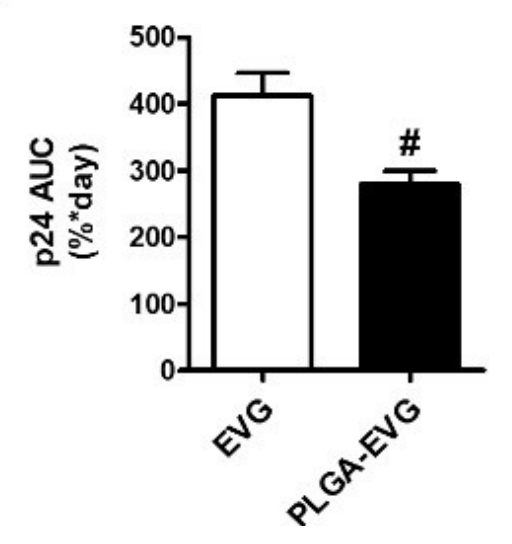

Figure 4-14. Viral suppression of EVG and PLGA-EVG in HIV-1-infected MMG after crossing an in vitro BBB model. (A) Treatment paradigm for collecting HIV-1-infected MMG and performing an EVG/PLGA-EVG viral suppression study. (B) TEER of the endothelial monolayer was measured at indicated time points. (C) Time course of HIV-1 replication upon drug exposure to HIV-1-infected MMG after crossing the in vitro BBB model. All assays were performed on triplicate samples with MMG derived from one donor. p24 levels were normalized to the control MMG, which were obtained from the same donor, and reported as a percentage of the control group. (D) Area under the curve of EVG and PLGA-EVG treatments. Area under the curve from 0 to last time $t(A U C 0-t)$ was calculated using the linear trapezoidal method. Mean \pm SEM values were graphed from triplicate samples with MMG derived from one donor. *indicates $\mathrm{p}<0.05$ compared to control, \#indicates $\mathrm{p}<0.05$ compared to EVG native drug. 


\section{CHAPTER 5. CONCLUSIONS AND FUTURE DIRECTIONS}

In this study, we developed a poloxamer-PLGA based NP loaded with EVG and showed the physicochemical characterization of this PLGA NP. Our current study demonstrated that this PLGA NP is an efficient delivery approach for EVG. The PLGAEVG NPs demonstrated a time- and concentration-dependent uptakes in monocytes and showed a higher intracellular internalization and superior viral suppression to EVG native drug in HIV-1-infected macrophages. The PLGA-EVG NPs demonstrated a favorable stability and safety profile. The PLGA-EVG NPs demonstrated significant transmigration across an in vitro $\mathrm{BBB}$, and an improved plasma drug concentration and drug brain concentrations in an in vivo mice model relative to EVG alone. PLGA-EVG NPs showed an improvement in viral suppression in both HIV-1-infected MDM and HIV-1-infected MMG after crossing the in vitro BBB compared with EVG native drug. PLGA-EVG NPs also showed a trend of decreasing viral load in the CNS in the humanized HIVE mice model compared with EVG native drug. To the best of our knowledge, this is the first report of using EVG nanoformulation as a potential delivery method to cross the BBB and suppress HIV-1 in the CNS. Moreover, compared to magnetic NPs, our PLGA-EVG NPs are easy to prepare, composed of FDA-approved non-toxic NPs, and do not require the application of an external magnetic field. We expect that PLGA-EVG NPs have the potential to move forward toward clinical use.

There were less than 10 studies documented using the nanoformulated ARVs according to clinical trial database (https://clinicaltrials.gov), ARVs such as efavirenz, lopinavir, cabotegravir and rilpivirine were involved in clinical trials that includes phase I/II/III [146]. A clinical trial (NCT02631473) conducted by St Stephens Aids Trust and University of Liverpool showed that a $50 \%$ dose reduction can be achieved by using for NPs for efavirenz and lopinavir compared to native drug regimens in healthy volunteers [146]. Additionally, a long-acting form of rilpivirine of nanosuspension made with poloxamer 338 and PEG 1000 has been investigated for safety profile in a phase II study (NCT02165202) [147]. In this study, one patient died, and 4 patients encountered with severe side effects among the 91 patients. Additionally, long acting nanoforumlation was also used with an integrase inhibitor cabotegravir and conducted a randomized clinical trial (NCT02076178), and showed a good safety, tolerability, and acceptability profiles in HIV patients with serious adverse effect observed in only one among the 124 patients [148]. The long-acting NPs with a combination of rilpivirine and cabotegravir showed a prolong HIV-1 inhibition till 96 weeks [149], and a good safety and tolerability profiles [150].

In our ongoing pilot experiment, we tested the PLGA-EVG NPs in an HIV-1 encephalitis (HIVE) mouse model where HIV-1-ADA-infected MDM were injected bilaterally into the basal ganglia of NSG humanized mice as described previously [151]. HIVE mouse model has been previously used for studies of HIV-associated neuroinflammation. The HIVE mouse model is a macrophage/microglia-centric model that is more appropriate to use in this proposal, and can be used to study the contributions of HIV-infected human macrophages and/or microglia to HAND [152] PLGA-EVG NPs 
showed a trend of decreasing the CNS HIV-1 gag expression in humanized HIVE mice model, relative to EVG native drug (Figure 5-1). Due to the limited animal number, we could not show a significant suppression in the CNS viral load using PLGA-EVG NPs. A further investigation with a higher number of animals can be conducted in the future to validate the efficacy of PLGA-EVG NPs in suppressing CNS viral load and improving cognitive dysfunction in HIV-1-infected humanized mice.

Recently, a new in vivo mouse model which reproduced many important characteristics of HIV has been developed. Humanized mice with CD34+ hematopoietic progenitor cell transplanted were infected by HIV-1 $1_{\mathrm{ADA}}$ strain. This CD34+ mice model represented human microglia-like cells in the mouse brain, that can be used to study CNS viral reservoir microglia, including CNS HIV-1 viral load and the effects of therapeutic interventions. In order to move forward, the efficacy of our nanoformulation can also be validated using this new mouse model to study the neurotoxicity and the effects of therapeutic interventions in mouse brain [153].

The future direction of this project can also be utilization of monoclonal antibodies to target delivery of NPs to macrophages and microglia. NPs with specific recognition ligands bound to the surface have good potential for targeted delivery. Targeted NPs have shown promise in improvement to cancer therapy alongside conventional chemotherapy [154]. Based on the studies in cancer therapy, using targeted NPs to deliver ARVs to viral reservoirs may offer advantages such as increased viral suppression, and minimized off-target toxicity [155]. The potential receptors for macrophages targeting can be the CD14 and CD16 receptors. Studies have shown that the number of CD14+ and CD16+ monocytes were higher in the peripheral blood of HIV-1infected individuals compared with uninfected subjects [156]. CD14+ and CD16+ monocytes are the subset of monocytes that are most permissive to HIV-1 infection, and preferentially transmigrate across the BBB [157-159]. The potential receptors for targeting NPs to microglia can be receptors Iba1 and CD68, which are highly expressed in microglia [160]. Another potential receptor for microglia targeting is transmembrane protein 119 (TMEM119). Recently, TMEM119 is gaining importance as a specific marker of microglia, because TMEM119 immunoreactivity was found to be expressed exclusively on $\mathrm{Iba}^{+} \mathrm{CD} 68^{+}$microglia in the human brain but not on $\mathrm{Iba} 1^{+} \mathrm{CD} 68^{+}$ infiltrating macrophages [161]. Therefore, targeting TMEM119 receptor to deliver the ARVs would be a potential way to suppress the virus specifically in microglia. However, after coupling with antibodies, PLGA NPs are likely to increase their particle size due to the large molecular weight of the added antibodies. The alternative way is to use monocytes or macrophages as endogenous drug carriers to deliver the NPs to the brain. Studies reported that monocytes and macrophages can cross the BBB under normal physiological conditions [162]. Previous studies have also attempted to use monocyte and macrophage-based drug carriers for targeted delivery of nanoformulated ART drugs to the brain $[163,164]$. For example, indinavir NPs which loaded on murine bone marrow macrophages, showed an increased drug concentration levels and a reduced HIV-1 replication in mouse brain using a HIVE mouse model [163]. Magnetic azidothymidine NPs loaded on monocytes showed an enhanced trans-endothelial migration compared to non-monocytes loaded NPs [164]. 


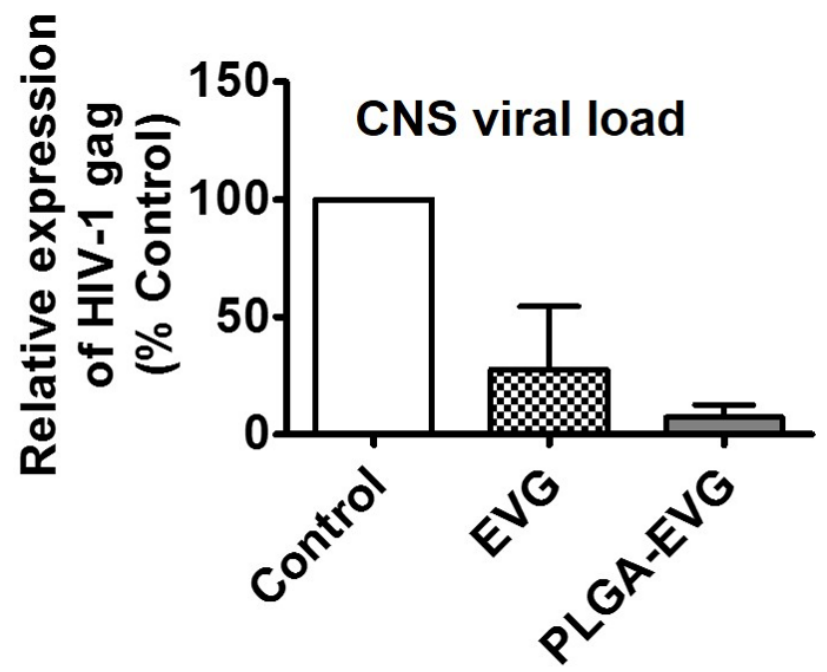

Figure 5-1. Viral suppression of EVG and PLGA-EVG in a HIVE mouse model. Viral suppression of EVG was measured after i.p. administration of $200 \mathrm{mg} / \mathrm{kg}$ dose of vehicle control (PLGA-Blank NPs, $\mathrm{n}=2)$, EVG native drug $(\mathrm{n}=2)$, or PLGA-EVG $(\mathrm{n}=$ 2) to HIVE mice. CNS viral load was presented as HIV-gag level and normalized to the control group, which was injected i.p. with PLGA-Blank NPs, and reported as a percentage of the control group. 
To further enhance the efficacy of the PLGA-EVG NPs, a commonly used combination of ARVs such as tenofovir alafenamide and emtricitabine can also be formulated with EVG into the same formulation to suppress different stages of the HIV-1 life cycle. Formulation of multiple drugs into PLGA NP is practical, and EVG has been used together with tenofovir alafenamide in a PLGA-based NPs coated with poloxamer 407 as an HIV-1 prevention strategy in a recent report [70]. However, introducing multiple ARVs to the CNS may also increase the potential of neurotoxicity. An alternative option is to use the chemodietary agent curcumin as an adjuvant therapy, which is being used in treating CNS diseases and reducing inflammation and oxidative stress, which are the hallmark of HIV-1 pathogenesis [165-167]. Curcumin supplement has been enrolled in 15 clinical phase I/II studies for the therapeutic intervention of cognitive impairment [167]. Curcumin has been described as an inhibitor of P-gp and CYP 3A4 $[168,169]$. Thus, in addition to its own anti-inflammatory and anti-oxidative effects, the use of curcumin will also aid in reducing the necessary EVG dose and prevent EVG-induced neurotoxicity.

Overall, nanotechnology is likely to provide a new approach for delivery of ARV for the intervention of HAND. Since nanotechnology is a novel delivery method, longterm adverse reactions, systemic toxicities, neurotoxicities, and the accumulation of nanomaterials in body need to be studied. In order to move forward to the clinical use, comprehensive toxicity and safety assessments of nanoformulation need to be addressed including animal studies using non-human primates to demonstrate efficacy, safety, and dose ranges. 


\section{LIST OF REFERENCES}

1. Hong, F.F. and J.W. Mellors, Changes in HIV reservoirs during long-term antiretroviral therapy. Current opinion in HIV and AIDS, 2015. 10(1): p. 43-48.

2. Saylor, D., et al., HIV-associated neurocognitive disorder - pathogenesis and prospects for treatment. Nature reviews. Neurology, 2016. 12(4): p. 234-248.

3. Maschke, M., et al., Incidence and prevalence of neurological disorders associated with HIV since the introduction of highly active antiretroviral therapy (HAART). Journal of Neurology, Neurosurgery, and Psychiatry, 2000. 69(3): $\mathrm{p}$. 376-380.

4. Verma, A.S., et al., Contribution of CNS cells in NeuroAIDS. Journal of Pharmacy and Bioallied Sciences, 2010. 2(4): p. 300-306.

5. Hazleton, J.E., J.W. Berman, and E.A. Eugenin, Novel mechanisms of central nervous system damage in HIV infection. HIV/AIDS (Auckland, N.Z.), 2010. 2: p. 39-49.

6. Kedzierska, K. and S.M. Crowe, The role of monocytes and macrophages in the pathogenesis of HIV-1 infection. Current medicinal chemistry, 2002. 9(21): $\mathrm{p}$. 1893-1903.

7. Burdo, T.H., A. Lackner, and K.C. Williams, Monocyte/macrophages and their role in HIV neuropathogenesis. Immunological reviews, 2013. 254(1): p. 102113.

8. Koenig, S., et al., Detection of AIDS virus in macrophages in brain tissue from AIDS patients with encephalopathy. Science, 1986. 233(4768): p. 1089-1093.

9. Wiley, C.A., et al., Cellular localization of human immunodeficiency virus infection within the brains of acquired immune deficiency syndrome patients. Proceedings of the National Academy of Sciences of the United States of America, 1986. 83(18): p. 7089-7093.

10. Castellano, P., L. Prevedel, and E.A. Eugenin, HIV-infected macrophages and microglia that survive acute infection become viral reservoirs by a mechanism involving Bim. Scientific Reports, 2017. 7(1): p. 12866.

11. Mocchetti, I., A. Bachis, and V. Avdoshina, Neurotoxicity of human immunodeficiency virus-1: viral proteins and axonal transport. Neurotoxicity research, 2012. 21(1): p. 79-89.

12. Kovalevich, J. and D. Langford, Neuronal toxicity in HIV CNS disease. Future virology, 2012. 7(7): p. 687-698.

13. Clifford, D.B. and B.M. Ances, HIV-Associated Neurocognitive Disorder (HAND). The Lancet infectious diseases, 2013. 13(11): p. 976-986.

14. Fois, A.F. and B.J. Brew, The Potential of the CNS as a Reservoir for HIV-I Infection: Implications for HIV Eradication. Current HIV/AIDS Reports, 2015. 12(2): p. 299-303.

15. Heaton, R.K., et al., HIV-associated neurocognitive disorders persist in the era of potent antiretroviral therapy: CHARTER Study. Neurology, 2010. 75(23): $\mathrm{p}$. 2087-2096.

16. Lange, J. and J. Ananworanich, The discovery and development of antiretroviral agents. Antivir Ther, 2014. 19(Suppl 3): p. 5-14. 
17. Mayer, K.H. and K.K. Venkatesh, Antiretroviral therapy as HIV prevention: status and prospects. American journal of public health, 2010. 100(10): p. 18671876.

18. FDA. Antiretroviral drugs used in the treatment of HIV infection. 2018 [cited 2018 11/20]; Available from: https://www.fda.gov/forpatients/illness/hivaids/treatment/ucm118915.htm.

19. De Clercq, E., Antiretroviral drugs. Current Opinion in Pharmacology, 2010. 10(5): p. 507-515.

20. De Clercq, E., Anti-HIV drugs: 25 compounds approved within 25 years after the discovery of HIV. International Journal of Antimicrobial Agents, 2009. 33(4): p. 307-320.

21. Yilmaz, A., R.W. Price, and M. Gisslén, Antiretroviral drug treatment of CNS HIV-1 infection. Journal of Antimicrobial Chemotherapy, 2012. 67(2): p. 299311.

22. Decloedt, E.H., et al., Central Nervous System Penetration of Antiretroviral Drugs: Pharmacokinetic, Pharmacodynamic and Pharmacogenomic Considerations. Clinical Pharmacokinetics, 2015. 54(6): p. 581-598.

23. Löscher, W. and H. Potschka, Blood-Brain Barrier Active Efflux Transporters: ATP-Binding Cassette Gene Family. NeuroRx, 2005. 2(1): p. 86-98.

24. Tran, N., Blood-brain barrier, in Encyclopedia of Clinical Neuropsychology. 2011, Springer. p. 426-426.

25. Gong, Y., et al., Pharmacokinetics and pharmacodynamics of cytochrome P450 inhibitors for HIV treatment. Expert Opinion on Drug Metabolism \& Toxicology, 2019. 15(5): p. 417-427.

26. Haas, D.W., et al., Effects of ritonavir on indinavir pharmacokinetics in cerebrospinal fluid and plasma. Antimicrobial agents and chemotherapy, 2003. 47(7): p. 2131-2137.

27. Letendre, S., et al., Validation of the CNS Penetration-Effectiveness Rank for Quantifying Antiretroviral Penetration Into the Central Nervous System. Archives of neurology, 2008. 65(1): p. 65-70.

28. Hammond, E.R., et al., The Cerebrospinal Fluid HIV Risk Score for Assessing Central Nervous System Activity in Persons With HIV. American Journal of Epidemiology, 2014. 180(3): p. 297-307.

29. Shapshak, P., et al., Editorial neuroAIDS review. AIDS (London, England), 2011. 25(2): p. 123-141.

30. Sacktor, N., et al., Paroxetine and fluconazole therapy for HIV-associated neurocognitive impairment: results from a double-blind, placebo-controlled trial. Journal of neurovirology, 2018. 24(1): p. 16-27.

31. Nair, M., et al., Getting into the Brain: Potential of Nanotechnology in the Management of NeuroAIDS. Advanced drug delivery reviews, 2016. 103: p. 202217.

32. Choo, E.F., et al., Pharmacological Inhibition of P-glycoprotein Transport Enhances the Distribution of HIV-1 Protease Inhibitors into Brain and Testes. Drug Metabolism and Disposition, 2000. 28(6): p. 655. 
33. Megard, I., et al., A co-culture-based model of human blood-brain barrier: application to active transport of indinavir and in vivo-in vitro correlation. Brain Research, 2002. 927(2): p. 153-167.

34. Park, S. and P.J. Sinko, P-Glycoprotein and Mutlidrug Resistance-Associated Proteins Limit the Brain Uptake of Saquinavir in Mice. Journal of Pharmacology and Experimental Therapeutics, 2005. 312(3): p. 1249.

35. Neuwelt, E.A., S.A. Hill, and E.P. Frenkel, Osmotic blood-brain barrier modification and combination chemotherapy: concurrent tumor regression in areas of barrier opening and progression in brain regions distant to barrier opening. Neurosurgery, 1984. 15(3): p. 362-366.

36. Qin, L.-J., et al., Bradykinin-induced blood-tumor barrier opening is mediated by tumor necrosis factor- $\alpha$. Neuroscience Letters, 2009. 450(2): p. 172-175.

37. Anthonypillai, C., J.E. Gibbs, and S.A. Thomas, The distribution of the anti-HIV drug, tenofovir (PMPA), into the brain, CSF and choroid plexuses. Cerebrospinal fluid research, 2006. 3: p. 1-1.

38. Sagar, V., et al., Towards nanomedicines for neuro-AIDS. Reviews in medical virology, 2014. 24(2): p. 103-124.

39. Wong, H.L., X.Y. Wu, and R. Bendayan, Nanotechnological advances for the delivery of CNS therapeutics. Advanced Drug Delivery Reviews, 2012. 64(7): p. 686-700.

40. Adhikary, R.R., P. More, and R. Banerjee, Smart nanoparticles as targeting platforms for HIV infections. Nanoscale, 2015. 7(17): p. 7520-7534.

41. Patel, T., et al., Polymeric nanoparticles for drug delivery to the central nervous system. Advanced drug delivery reviews, 2012. 64(7): p. 701-705.

42. Zhou, Y., et al., Crossing the blood-brain barrier with nanoparticles. Journal of Controlled Release, 2018. 270: p. 290-303.

43. Koushik, O., et al., Nano drug delivery systems to overcome cancer drug resistance-a review. J Nanomed Nanotechnol, 2016. 7(378): p. 2.

44. Shi, J., et al., Cancer nanomedicine: progress, challenges and opportunities. Nature Reviews Cancer, 2016. 17: p. 20.

45. Jianxiang, Z., L. Shuhui, and L. Xiaohui, Polymeric Nano-Assemblies as Emerging Delivery Carriers for Therapeutic Applications: A Review of Recent Patents. Recent Patents on Nanotechnology, 2009. 3(3): p. 225-231.

46. Kamaly, N., et al., Targeted polymeric therapeutic nanoparticles: design, development and clinical translation. Chemical Society Reviews, 2012. 41(7): p. 2971-3010.

47. Roy, U., et al., The potential of HIV-1 nanotherapeutics: from in vitro studies to clinical trials. Nanomedicine (Lond), 2015. 10(24): p. 3597-609.

48. Shao, J., et al., Nanodrug formulations to enhance HIV drug exposure in lymphoid tissues and cells: clinical significance and potential impact on treatment and eradication of HIV/AIDS. Nanomedicine (Lond), 2016. 11(5): p. 545-64.

49. Edagwa, B.J., et al., Development of HIV reservoir targeted long acting nanoformulated antiretroviral therapies. Curr Med Chem, 2014. 21(36): p. 418698. 
50. Vyas, T.K., L. Shah, and M.M. Amiji, Nanoparticulate drug carriers for delivery of HIV/AIDS therapy to viral reservoir sites. Expert Opinion on Drug Delivery, 2006. 3(5): p. 613-628.

51. Kuo, Y.-C. and F.-L. Su, Transport of stavudine, delavirdine, and saquinavir across the blood-brain barrier by polybutylcyanoacrylate, methylmethacrylatesulfopropylmethacrylate, and solid lipid nanoparticles. International Journal of Pharmaceutics, 2007. 340(1): p. 143-152.

52. Kuo, Y.-C. and C.-L. Lee, Methylmethacrylate-sulfopropylmethacrylate nanoparticles with surface RMP-7 for targeting delivery of antiretroviral drugs across the blood-brain barrier. Colloids and Surfaces B: Biointerfaces, 2012. 90: p. 75-82.

53. Kuo, Y.-C. and H.-H. Chen, Effect of nanoparticulate polybutylcyanoacrylate and methylmethacrylate-sulfopropylmethacrylate on the permeability of zidovudine and lamivudine across the in vitro blood-brain barrier. International Journal of Pharmaceutics, 2006. 327(1): p. 160-169.

54. Mainardes, R.M., et al., Zidovudine-loaded PLA and PLA-PEG blend nanoparticles: Influence of polymer type on phagocytic uptake by polymorphonuclear cells. Journal of Pharmaceutical Sciences, 2009. 98(1): p. 257-267.

55. Saiyed, Z.M., N.H. Gandhi, and M.P.N. Nair, Magnetic nanoformulation of azidothymidine 5'-triphosphate for targeted delivery across the blood-brain barrier. International Journal of Nanomedicine, 2010. 5: p. 157-166.

56. Jayant, R.D., et al., Sustained-release nanoART formulation for the treatment of neuroAIDS. International journal of nanomedicine, 2015. 10: p. 1077-1093.

57. Ding, H., et al., Enhanced blood-brain barrier transmigration using a novel transferrin embedded fluorescent magneto-liposome nanoformulation. Nanotechnology, 2014. 25(5): p. 055101-055101.

58. Jayant, R.D., et al., Multifunctional Nanotherapeutics for the Treatment of neuroAIDS in Drug Abusers. Scientific reports, 2018. 8(1): p. 12991-12991.

59. Nair, M., et al., Externally controlled on-demand release of anti-HIV drug using magneto-electric nanoparticles as carriers. Nature Communications, 2013. 4(1): p. 1707.

60. Tomitaka, A., et al., Hybrid magneto-plasmonic liposomes for multimodal imageguided and brain-targeted HIV treatment. Nanoscale, 2018. 10(1): p. 184-194.

61. Desai, J. and H. Thakkar, Effect of particle size on oral bioavailability of darunavir-loaded solid lipid nanoparticles. Journal of microencapsulation, 2016. 33(7): p. 669-678.

62. Desai, J. and H. Thakkar, Darunavir-Loaded Lipid Nanoparticles for Targeting to HIV Reservoirs. AAPS PharmSciTech, 2018. 19(2): p. 648-660.

63. Vyas, T.K., A. Shahiwala, and M.M. Amiji, Improved oral bioavailability and brain transport of Saquinavir upon administration in novel nanoemulsion formulations. International journal of pharmaceutics, 2008. 347(1-2): p. 93-101.

64. Montenegro-Burke, J.R., et al., Nanoformulated antiretroviral therapy attenuates brain metabolic oxidative stress. Molecular Neurobiology, 2019. 56(4): p. 28962907. 
65. Thomsen, L.B., M.S. Thomsen, and T. Moos, Targeted drug delivery to the brain using magnetic nanoparticles. Therapeutic Delivery, 2015. 6(10): p. 1145-1155.

66. Ventola, C.L., Progress in nanomedicine: approved and investigational nanodrugs. Pharmacy and Therapeutics, 2017. 42(12): p. 742.

67. Messam, C.A. and E.O. Major, Stages of restricted HIV-1 infection in astrocyte cultures derived from human fetal brain tissue. Journal of neurovirology, 2000. 6(1): p. S90.

68. Unger, N.R., et al., Elvitegravir for the treatment of HIV. Expert Opin Pharmacother, 2016. 17(17): p. 2359-2370.

69. Mohideen, M., et al., Degradable bioadhesive nanoparticles for prolonged intravaginal delivery and retention of elvitegravir. Biomaterials, 2017. 144: p. 144-154.

70. Mandal, S., et al., Tenofovir alafenamide and elvitegravir loaded nanoparticles for long-acting prevention of HIV-1 vaginal transmission. AIDS, 2017. 31(4): p. 469-476.

71. Prathipati, P.K., et al., Pharmacokinetic and Tissue Distribution Profile of Long Acting Tenofovir Alafenamide and Elvitegravir Loaded Nanoparticles in Humanized Mice Model. Pharmaceutical Research, 2017. 34(12): p. 2749-2755.

72. Pommier, Y., A.A. Johnson, and C. Marchand, Integrase inhibitors to treat HIV/Aids. Nat Rev Drug Discov, 2005. 4(3): p. 236-248.

73. Calcagno, A., et al., Elvitegravir/Cobicistat/Tenofovir/Emtricitabine Penetration in the Cerebrospinal Fluid of Three HIV-Positive Patients. AIDS Research and Human Retroviruses, 2016. 32(5): p. 409-411.

74. Makadia, H.K. and S.J. Siegel, Poly Lactic-co-Glycolic Acid (PLGA) as Biodegradable Controlled Drug Delivery Carrier. Polymers, 2011. 3(3): p. 13771397.

75. Cai, Q., et al., Systemic delivery to central nervous system by engineered PLGA nanoparticles. American journal of translational research, 2016. 8(2): p. 749-764.

76. Vivithanaporn, P., M.J. Gill, and C. Power, Impact of current antiretroviral therapies on neuroAIDS. Expert review of anti-infective therapy, 2011. 9(4): p. 371-374.

77. Lee, G., et al., Functional Expression of P-glycoprotein in Rat Brain Microglia. Journal of Pharmacology and Experimental Therapeutics, 2001. 299(1): p. 204.

78. Woodland, C., et al., Expression, Activity and Regulation of CYP3A in Human and Rodent Brain. Drug Metabolism Reviews, 2008. 40(1): p. 149-168.

79. Asahchop, E.L., et al., Reduced antiretroviral drug efficacy and concentration in $H I V$-infected microglia contributes to viral persistence in brain. Retrovirology, 2017. 14: p. 47.

80. Gelperina, S., et al., Drug delivery to the brain using surfactant-coated poly(lactide-co-glycolide) nanoparticles: Influence of the formulation parameters. European Journal of Pharmaceutics and Biopharmaceutics, 2010. 74(2): p. 157163.

81. Gong, Y., et al., Novel elvitegravir nanoformulation approach to suppress the viral load in HIV-infected macrophages. Biochemistry and Biophysics Reports, 2017. 12: p. 214-219. 
82. Gong, Y., et al., Novel elvitegravir nanoformulation for drug delivery across the blood-brain barrier to achieve HIV-1 suppression in the CNS macrophages.

Scientific Reports, 2020. 10(1): p. 3835.

83. Yallapu, M.M., et al., Fabrication of curcumin encapsulated PLGA nanoparticles for improved therapeutic effects in metastatic cancer cells. J Colloid Interface Sci, 2010. 351(1): p. 19-29.

84. Etemad, S., et al., A novel in vitro human microglia model: Characterization of human monocyte-derived microglia. Journal of Neuroscience Methods, 2012. 209(1): p. 79-89.

85. Ranjit, S., et al., Effect of Polyaryl Hydrocarbons on Cytotoxicity in Monocytic Cells: Potential Role of Cytochromes P450 and Oxidative Stress Pathways. PLoS ONE, 2016. 11(9): p. e0163827.

86. Midde, N.M., et al., Effect of Ethanol on the Metabolic Characteristics of HIV-1 Integrase Inhibitor Elvitegravir and Elvitegravir/Cobicistat with CYP3A: An Analysis Using a Newly Developed LC-MS/MS Method. PloS one, 2016. 11(2): p. e0149225-e0149225.

87. Yallapu, M.M., et al., Implications of protein corona on physico-chemical and biological properties of magnetic nanoparticles. Biomaterials, 2015. 46: p. 1-12.

88. Helms, H.C., et al., In vitro models of the blood-brain barrier: An overview of commonly used brain endothelial cell culture models and guidelines for their use. Journal of Cerebral Blood Flow \& Metabolism, 2016. 36(5): p. 862-890.

89. Shi, D., et al., Optimizing superparamagnetic iron oxide nanoparticles as drug carriers using an in vitro blood-brain barrier model. International Journal of Nanomedicine, 2016. 11: p. 5371-5379.

90. Srinivasan, B., et al., TEER measurement techniques for in vitro barrier model systems. Journal of laboratory automation, 2015. 20(2): p. 107-126.

91. Joyce, A.P., et al., One Mouse, One Pharmacokinetic Profile: Quantitative Whole Blood Serial Sampling for Biotherapeutics. Pharmaceutical Research, 2014. 31(7): p. 1823-1833.

92. Aquaro, S., et al., Mechanisms underlying activity of antiretroviral drugs in HIV1-infected macrophages: new therapeutic strategies. J Leukoc Biol, 2006. 80(5): p. 1103-10.

93. Kumar, A., W. Abbas, and G. Herbein, HIV-1 Latency in Monocytes/Macrophages. Viruses, 2014. 6(4): p. 1837-1860.

94. Bazzoli, C., et al., Intracellular Pharmacokinetics of Antiretroviral Drugs in HIVInfected Patients, and their Correlation with Drug Action. Clin Pharmacokinet, 2010. 49(1): p. 17-45.

95. Jin, M., et al., Effect of alcohol on drug efflux protein and drug metabolic enzymes in U937 macrophages. Alcohol Clin Exp Res, 2011. 35(1): p. 132-139.

96. Cory, T.J., et al., Alterations in P-Glycoprotein Expression and Function Between Macrophage Subsets. Pharmaceutical Research, 2016. 33(11): p. 2713-2721.

97. Perno, C.F., et al., Relative potency of protease inhibitors in monocytes/macrophages acutely and chronically infected with human immunodeficiency virus. J Infect Dis, 1998. 178(2): p. 413-422. 
98. Scopelliti, F., et al., Comparative antiviral activity of integrase inhibitors in human monocyte-derived macrophages and lymphocytes. Antiviral Research, 2011. 92(2): p. 255-261.

99. Konig, S.K., et al., Impact of drug transporters on cellular resistance towards saquinavir and darunavir. J Antimicrob Chemother, 2010. 65(11): p. 2319-28.

100. Iyidogan, P. and K.S. Anderson, Current Perspectives on HIV-1 Antiretroviral Drug Resistance. Viruses, 2014. 6(10): p. 4095-4139.

101. Klibanov, O.M., Elvitegravir, an oral HIV integrase inhibitor, for the potential treatment of HIV infection. Curr Opin Investig Drugs, 2009. 10(2): p. 190-200.

102. Pandey, K.K., Critical appraisal of elvitegravir in the treatment of HIV-1/AIDS. HIV/AIDS (Auckland, N.Z.), 2014. 6: p. 81-90.

103. Honary, S. and F. Zahir, Effect of zeta potential on the properties of nano-drug delivery systems-a review (Part 1). Tropical Journal of Pharmaceutical Research, 2013. 12(2): p. 255-264.

104. Yallapu, M.M., et al., Curcumin-loaded magnetic nanoparticles for breast cancer therapeutics and imaging applications. International Journal of Nanomedicine, 2012. 7: p. 1761-1779.

105. Pirooznia, N., et al., Encapsulation of Alpha-1 antitrypsin in PLGA nanoparticles: In Vitro characterization as an effective aerosol formulation in pulmonary diseases. Journal of Nanobiotechnology, 2012. 10: p. 20-20.

106. Lü, J.-M., et al., Current advances in research and clinical applications of PLGAbased nanotechnology. Expert review of molecular diagnostics, 2009. 9(4): p. 325-341.

107. Fonseca, C., S. Simoes, and R. Gaspar, Paclitaxel-loaded PLGA nanoparticles: preparation, physicochemical characterization and in vitro anti-tumoral activity. J Control Release, 2002. 83.

108. Su, W.-P., et al., PLGA nanoparticles codeliver paclitaxel and Stat 3 siRNA to overcome cellular resistance in lung cancer cells. International Journal of Nanomedicine, 2012. 7: p. 4269-4283.

109. Khan, S., et al., Nanoparticle formulation of ormeloxifene for pancreatic cancer. Biomaterials, 2015. 53: p. 731-743.

110. Zaman, M.S., et al., Curcumin Nanoformulation for Cervical Cancer Treatment. Scientific Reports, 2016. 6: p. 20051.

111. Jain, R.A., The manufacturing techniques of various drug loaded biodegradable poly(lactide-co-glycolide) (PLGA) devices. Biomaterials, 2000. 21(23): p. 24752490.

112. Abdelbary, A.A., et al., Preparation, optimization, and in vitro simulated inhalation delivery of carvedilol nanoparticles loaded on a coarse carrier intended for pulmonary administration. International Journal of Nanomedicine, 2015. 10: p. 6339-6353.

113. Pasche, S., et al., Effects of Ionic Strength and Surface Charge on Protein Adsorption at PEGylated Surfaces. The Journal of Physical Chemistry B, 2005. 109(37): p. 17545-17552.

114. Janneh, O., et al., Modulation of the intracellular accumulation of saquinavir in peripheral blood mononuclear cells by inhibitors of MRP1, MRP2, P-gp and BCRP. AIDS, 2005. 19(18): p. 2097-102. 
115. Turriziani, O., et al., Expression levels of MDR1, MRP1, MRP4, and MRP5 in peripheral blood mononuclear cells from HIV infected patients failing antiretroviral therapy. Journal of Medical Virology, 2008. 80(5): p. 766-771.

116. Kolars, J.C., et al., CYP3A gene expression in human gut epithelium. Pharmacogenetics and Genomics, 1994. 4(5): p. 247-259.

117. Tang, J., et al., Solid lipid nanoparticles with TPGS and Brij 78: A co-delivery vehicle of curcumin and piperine for reversing $P$-glycoprotein-mediated multidrug resistance in vitro. Oncology Letters, 2017. 13(1): p. 389-395.

118. Rao, K.S., et al., TAT-conjugated nanoparticles for the CNS delivery of anti-HIV drugs. Biomaterials, 2008. 29(33): p. 4429-4438.

119. Chavanpatil, M.D., et al., Surfactant-Polymer Nanoparticles Overcome PGlycoprotein-Mediated Drug Efflux. Molecular Pharmaceutics, 2007. 4(5): p. 730-738.

120. Joshi, G., A. Kumar, and K. Sawant, Bioavailability enhancement, Caco-2 cells uptake and intestinal transport of orally administered lopinavir-loaded PLGA nanoparticles. Drug Delivery, 2016. 23(9): p. 3492-3504.

121. Josephson, F., Drug-drug interactions in the treatment of HIV infection: focus on pharmacokinetic enhancement through CYP3A inhibition. Journal of Internal Medicine, 2010. 268(6): p. 530-539.

122. Kumar, S., et al., Drug-drug interactions between anti-retroviral therapies and drugs of abuse in HIV systems. Expert opinion on drug metabolism \& toxicology, 2015. 11(3): p. 343-355.

123. Rahman, M., et al., Nanoparticle and protein corona, in Protein-nanoparticle interactions. 2013, Springer. p. 21-44.

124. Neagu, M., et al., Protein bio-corona: critical issue in immune nanotoxicology. Archives of toxicology, 2017. 91(3): p. 1031-1048.

125. Mittal, P., et al., Formulation, optimization, hemocompatibility and pharmacokinetic evaluation of PLGA nanoparticles containing paclitaxel. Drug Development and Industrial Pharmacy, 2019. 45(3): p. 365-378.

126. Masserini, M., Nanoparticles for brain drug delivery. ISRN biochemistry, 2013. 2013.

127. Nowak, M., et al., Size, shape, and flexibility influence nanoparticle transport across brain endothelium under flow. Bioengineering \& Translational Medicine, 2019. n/a(n/a): p. e10153.

128. Anderson, L. and N.G. Anderson, High resolution two-dimensional electrophoresis of human plasma proteins. Proceedings of the National Academy of sciences, 1977. 74(12): p. 5421-5425.

129. Zhang, Z., et al., Brain-targeted drug delivery by manipulating protein corona functions. Nature Communications, 2019. 10(1): p. 3561.

130. Morais, J.M., F. Papadimitrakopoulos, and D.J. Burgess, Biomaterials/tissue interactions: possible solutions to overcome foreign body response. The AAPS journal, 2010. 12(2): p. 188-196.

131. Yallapu, M.M., et al., Anti-cancer activity of curcumin loaded nanoparticles in prostate cancer. Biomaterials, 2014. 35(30): p. 8635-8648. 
132. Zhang, L., et al., Cytosolic co-delivery of miRNA-34a and docetaxel with coreshell nanocarriers via caveolae-mediated pathway for the treatment of metastatic breast cancer. Scientific reports, 2017. 7: p. 46186-46186.

133. Iversen, T.-G., T. Skotland, and K. Sandvig, Endocytosis and intracellular transport of nanoparticles: Present knowledge and need for future studies. Nano Today, 2011. 6(2): p. 176-185.

134. Selby, L.I., et al., Nanoescapology: progress toward understanding the endosomal escape of polymeric nanoparticles. Wiley Interdisciplinary Reviews: Nanomedicine and Nanobiotechnology, 2017. 9(5): p. e1452.

135. Papa, S., et al., Polymeric nanoparticle system to target activated microglia/macrophages in spinal cord injury. Journal of Controlled Release, 2014. 174: p. 15-26.

136. Papa, S., et al., Selective Nanovector Mediated Treatment of Activated Proinflammatory Microglia/Macrophages in Spinal Cord Injury. ACS Nano, 2013. 7(11): p. 9881-9895.

137. Zhang, F., et al., Targeting specific cells in the brain with nanomedicines for CNS therapies. Journal of controlled release : official journal of the Controlled Release Society, 2016. 240: p. 212-226.

138. Kuhn, D.A., et al., Different endocytotic uptake mechanisms for nanoparticles in epithelial cells and macrophages. Beilstein journal of nanotechnology, 2014. 5: p. 1625-1636.

139. Oh, N. and J.-H. Park, Endocytosis and exocytosis of nanoparticles in mammalian cells. International journal of nanomedicine, 2014. 9 Suppl 1(Suppl 1): p. 51-63.

140. Kim, S. and I.-H. Choi, Phagocytosis and endocytosis of silver nanoparticles induce interleukin-8 production in human macrophages. Yonsei medical journal, 2012. 53(3): p. 654-657.

141. Yang, S., H. Jin, and Z. Zhao, Paracellular tightness and the functional expression of efflux transporters P-gp and BCRP in bEnd3 cells. Neurological Research, 2018: p. 1-6.

142. Omidi, Y., et al., Evaluation of the immortalised mouse brain capillary endothelial cell line, b.End3, as an in vitro blood-brain barrier model for drug uptake and transport studies. Brain Research, 2003. 990(1): p. 95-112.

143. Gong, Y., et al., Novel elvitegravir nanoformulation approach to suppress the viral load in HIV-infected macrophages. Biochemistry and Biophysics Reports, 2017. 12(Supplement C): p. 214-219.

144. Patel, H.R., R.P. Patel, and M. Patel, Poloxamers: A pharmaceutical excipients with therapeutic behaviors. International Journal of PharmTech Research, 2009. 1(2): p. 299-303.

145. Kulkarni, S.A. and S.-S. Feng, Effects of surface modification on delivery efficiency of biodegradable nanoparticles across the blood-brain barrier. Nanomedicine, 2011. 6(2): p. 377-394.

146. Caster, J.M., et al., Investigational nanomedicines in 2016: a review of nanotherapeutics currently undergoing clinical trials. Wiley Interdiscip Rev Nanomed Nanobiotechnol, 2017. 9(1).

147. Olivia, S., et al., Preclinical Evaluation of TMC-278 LA, a Long-acting Formulation of Rilpivirine, Demonstrates Significant Protection from Vaginal 
HIV Infection. AIDS Research and Human Retroviruses, 2014. 30(S1): p. A11A12.

148. ClinicalTrials Database: NCT02165202. ClinicalTrials.gov.

149. Margolis, D.A., et al., Long-acting intramuscular cabotegravir and rilpivirine in adults with HIV-1 infection (LATTE-2): 96-week results of a randomised, openlabel, phase 2b, non-inferiority trial. Lancet, 2017. 390(10101): p. 1499-1510.

150. Spreen, W., et al., Pharmacokinetics, safety, and tolerability with repeat doses of GSK1265744 and rilpivirine (TMC278) long-acting nanosuspensions in healthy adults. J Acquir Immune Defic Syndr, 2014. 67(5): p. 487-92.

151. Poluektova, L., et al., Neuroregulatory Events Follow Adaptive Immune-Mediated Elimination of HIV-1-Infected Macrophages: Studies in a Murine Model of Viral Encephalitis. The Journal of Immunology, 2004. 172(12): p. 7610-7617.

152. Jaeger, L.B. and A. Nath, Modeling HIV-associated neurocognitive disorders in mice: new approaches in the changing face of HIV neuropathogenesis. Disease Models \& Mechanisms, 2012. 5(3): p. 313-322.

153. Mathews, S., et al., Human Interleukin-34 facilitates microglia-like cell differentiation and persistent HIV-1 infection in humanized mice. Molecular Neurodegeneration, 2019. 14(1): p. 12.

154. Rosenblum, D., et al., Progress and challenges towards targeted delivery of cancer therapeutics. Nature Communications, 2018. 9(1): p. 1410.

155. Johnston, M.C. and C.J. Scott, Antibody conjugated nanoparticles as a novel form of antibody drug conjugate chemotherapy. Drug Discovery Today: Technologies, 2018. 30: p. 63-69.

156. Ziegler-Heitbrock, L., The CD14+CD16+ blood monocytes: their role in infection and inflammation. Journal of Leukocyte Biology, 2006. 81(3): p. 584592.

157. Williams, D.W., et al., Mechanisms of HIV entry into the CNS: increased sensitivity of HIV infected CD14+CD16+ monocytes to CCL2 and key roles of CCR2, JAM-A, and ALCAM in diapedesis. PloS one, 2013. 8(7): p. e69270.

158. Pulliam, L., et al., Unique monocyte subset in patients with AIDS dementia. The Lancet, 1997. 349(9053): p. 692-695.

159. Veenstra, M., et al., Mechanisms of CNS Viral Seeding by HIV+CD14+CD16+ Monocytes: Establishment and Reseeding of Viral Reservoirs Contributing to HIV-Associated Neurocognitive Disorders. mBio, 2017. 8(5): p. e01280-17.

160. Hendrickx, D.A.E., et al., Staining of HLA-DR, Ibal and CD68 in human microglia reveals partially overlapping expression depending on cellular morphology and pathology. Journal of Neuroimmunology, 2017. 309: p. 12-22.

161. Satoh, J.-i., et al., TMEM119 marks a subset of microglia in the human brain. Neuropathology, 2016. 36(1): p. 39-49.

162. Perry, V.H., et al., The blood-brain barrier and the inflammatory response. Molecular Medicine Today, 1997. 3(8): p. 335-341.

163. Dou, H., et al., Macrophage delivery of nanoformulated antiretroviral drug to the brain in a murine model of neuroAIDS. Journal of immunology (Baltimore, Md. : 1950), 2009. 183(1): p. 661-669. 
164. Saiyed, Z.M., N.H. Gandhi, and M.P.N. Nair, Magnetic nanoformulation of azidothymidine 5'-triphosphate for targeted delivery across the blood-brain barrier. International journal of nanomedicine, 2010. 5: p. 157-166.

165. Sorrenti, V., et al., Curcumin Prevents Acute Neuroinflammation and Long-Term Memory Impairment Induced by Systemic Lipopolysaccharide in Mice. Frontiers in pharmacology, 2018. 9: p. 183-183.

166. Lin, X., et al., Curcumin attenuates oxidative stress in RAW264.7 cells by increasing the activity of antioxidant enzymes and activating the Nrf2-Keap 1 pathway. PloS one, 2019. 14(5): p. e0216711-e0216711.

167. Prasad, S. and A.K. Tyagi, Curcumin and its analogues: a potential natural compound against HIV infection and AIDS. Food \& function, 2015. 6(11): p. 3412-3419.

168. Sasaki, T., et al., Effect of health foods on cytochrome P450-mediated drug metabolism. Journal of pharmaceutical health care and sciences, 2017. 3: p. 14-14.

169. Lopes-Rodrigues, V., E. Sousa, and M.H. Vasconcelos, Curcumin as a Modulator of P-Glycoprotein in Cancer: Challenges and Perspectives. Pharmaceuticals (Basel, Switzerland), 2016. 9(4): p. 71. 


\section{VITA}

Yuqing Gong, daughter of Li Gong and Hong Mao, was born in Kunming, China in 1992. She graduated from Kunming No1. High school, Kunming, China. She completed her undergraduate study in China Pharmaceutical University, Nanjing, China, and received Bachelor of Science degree in Chinese Pharmaceutical Science in 2014. She graduated from Temple University with a Master of Science degree in Pharmaceutical Science in 2016 in Philadelphia. The same year, she started her graduate study at The University of Tennessee Health Science Center to pursue a doctoral degree in Pharmaceutical Sciences in Memphis. She will be graduating in May 2020. 